\title{
A Gyrogeometric Mean in the Einstein Gyrogroup
}

\author{
Takuro Honma ${ }^{1}$ and Osamu Hatori ${ }^{2, *(D)}$ \\ 1 Niigata Pref. Takada Senior High School, Yasuduka Branch School, Yasuduka 942-0411, Japan; \\ tak.hon1090@gmail.com \\ 2 Institute of Science and Technology, Niigata University, Niigata 950-2181, Japan \\ * Correspondence: hatori@math.sc.niigata-u.ac.jp
}

Received: 20 July 2020; Accepted: 4 August 2020; Published: 10 August 2020

\begin{abstract}
In this paper, we define a gyrogeometric mean on the Einstein gyrovector space. It satisfies several properties one would expect for means. For example, it is permutation-invariant and left-translation invariant. It is already known that the Einstein gyrogroup is a gyrocommutative gyrogroup. We give an alternative proof which depends only on an elementary calculation.
\end{abstract}

Keywords: gyrogroup; Einstein velocity addition; gyrogeometric mean

\section{Introduction}

Einstein addition is a binary operation that stems from his velocity composition law of relativistic admissible velocities. Einstein addition is neither commutative nor associative. Ungar initiated the study of gyrogroups and gyrovector spaces [1] associated with the Einstein addition in the theory of special relativity. A gyrocommutative gyrogroup is a gyrogroup which has weak associativity and commutativity. It is a generalization of a commutative group.

Let $\mathbb{V}$ be a real inner product space. For a positive real number $s$ we denote $\mathbb{V}_{s}$ the s-ball of $\mathbb{V}$, i.e.,

$$
\mathbb{V}_{s}=\{\mathbf{v} \in \mathbb{V}:\|\mathbf{v}\|<s\}
$$

The Einstein addition $\oplus_{E}$ on $\mathbb{V}_{S}$ is a binary operation on $\mathbb{V}_{S}$ given by the equation

$$
\mathbf{u} \oplus_{E} \mathbf{v}=\frac{1}{1+\frac{\mathbf{u} \cdot \mathbf{v}}{s^{2}}}\left\{\mathbf{u}+\frac{1}{\gamma_{\mathbf{u}}} \mathbf{v}+\frac{1}{s^{2}} \frac{\gamma_{\mathbf{u}}}{1+\gamma_{\mathbf{u}}}(\mathbf{u} \cdot \mathbf{v}) \mathbf{u}\right\},
$$

where $\gamma_{\mathbf{u}}$ is the gamma factor

$$
\gamma_{\mathbf{u}}=\frac{1}{\sqrt{1-\frac{\|\mathbf{u}\|^{2}}{s^{2}}}}
$$

in $\mathbb{V}_{S}$, where $\cdot$ and $\|\cdot\|$ are the inner product and the norm of $\mathbb{V}$ respectively. By the definition of $\oplus_{E}$, $\mathbf{u} \oplus_{E} \mathbf{v} \in \mathbb{V}_{S}$ for every pair $\mathbf{u}, \mathbf{v} \in \mathbb{V}_{S}$ by Theorem 3.46 and the identity (3.189) in [1].

In [1] (p. 88) Ungar described that "one can show by computer algebra that Einstein addition in the ball is a gyrocommutative gyrogroup operation, giving rise to the Einstein ball gyrogroup $\left(\mathbb{V}_{1}, \oplus_{E}\right) .{ }^{\prime \prime}$ On the other hand, Suksumran and Wiboonton [2] gave a proof applying the theory of Clifford algebras, without using computer algebras. We give an elementary and direct proof in Section 6, which is lengthy but just by a simple calculation without applying any substantial theory of mathematics. 
In the following up to Section 5 we assume $s=1$ just for simplicity. The Einstein scalar multiplication $\otimes_{E}$ on $\left(\mathbb{V}_{1}, \oplus_{E}\right)$ is given by the equation

$$
\begin{aligned}
r \otimes_{E} \mathbf{a} & =\frac{(1+\|\mathbf{a}\|)^{r}-(1-\|\mathbf{a}\|)^{r}}{(1+\|\mathbf{a}\|)^{r}+(1-\|\mathbf{a}\|)^{r}} \frac{\mathbf{a}}{\|\mathbf{a}\|} \\
& =\tanh \left(r \tanh ^{-1}\|\mathbf{a}\|\right) \frac{\mathbf{a}}{\|\mathbf{a}\|},
\end{aligned}
$$

where $r \in \mathbb{R}, \mathbf{a} \in \mathbb{V}_{1} \backslash\{\mathbf{0}\} ;$ and $r \otimes_{E} \mathbf{0}=\mathbf{0}$. By Theorem 6.84 in [1], $\left(\mathbb{V}_{1}, \oplus_{E}, \otimes_{E}\right)$ is a gyrovector space, which is called an Einstein gyrovector space.

Ungar [1] (pp. 172-173) defined the gyromidpoint $\mathbf{P}_{\mathbf{a b}}^{m}$ of two elements in a gyrovector space. For $\mathbf{a}, \mathbf{b} \in \mathbb{V}_{1}$ we have

$$
\mathbf{P}_{\mathbf{a b}}^{m}=\frac{\gamma_{\mathbf{a}} \mathbf{a}+\gamma_{\mathbf{b}} \mathbf{b}}{\gamma_{\mathbf{a}}+\gamma_{\mathbf{b}}}
$$

Ungar also defined the gyrocentroid $\mathbb{C}_{\mathbf{a b c}}^{m}$ of three elements $\mathbf{a}, \mathbf{b}, \mathbf{c} \in \mathbb{V}_{1}$ as

$$
\mathbb{C}_{\mathbf{a b c}}^{m}=\frac{\gamma_{\mathbf{a}} \mathbf{a}+\gamma_{\mathbf{b}} \mathbf{b}+\gamma_{\mathbf{c}} \mathbf{c}}{\gamma_{\mathbf{a}}+\gamma_{\mathbf{b}}+\gamma_{\mathbf{c}}}
$$

The gyromidpoint corresponds to the average of two velocities in the special theory of relativity. On the other hand the gyrocentroid $\mathbb{C}_{\mathrm{abc}}^{m}$ does not satisfy a certain desirable property one would expected for means; by a simple calculation we have $\mathbb{C}_{\mathrm{abc}}^{m}=\frac{\gamma_{\mathbf{c}}}{\gamma_{\mathbf{c}}+2} \mathbf{c} \neq \frac{1}{3} \otimes_{E} \mathbf{c}$ for $\mathbf{a}=\mathbf{b}=\mathbf{0}$ and $\mathbf{c} \neq 0$. In this paper, we propose an alternative definition of the mean of three or more elements, the gyrogeometric mean, and show that it has several properties one would expect for means. The gyrogeometric mean corresponding to the average of the velocities in the special relativity. It is symmetric in the sense that permutation-invariant by the definition of the gyrogeometric mean. It is translation invariant (Proposition 5). The main idea of the definitions come from the geometric mean for positive definite matrices by Bhatia and Holbrook [3] and Ando, Li and Mathias [4].

2. The Metric Space $\left(\mathbb{V}_{1}, \oplus_{E}, \otimes_{E}\right)$

We define the set $\left\|\mathbb{V}_{1}\right\|=\left\{ \pm\|\mathbf{v}\|: \mathbf{v} \in \mathbb{V}_{1}\right\} \subset \mathbb{R}$ which coincides with the open interval $(-1,1)$. $\left\|\mathbb{V}_{1}\right\|$ admits the addition $\oplus^{\prime}$ and the scalar multiplication $\otimes^{\prime}$ given by the following:

$$
\begin{aligned}
a \oplus^{\prime} b & =\frac{a+b}{1+a b} \\
r \otimes^{\prime} a & =\tanh \left(r \tanh ^{-1} a\right)
\end{aligned}
$$

where $a, b \in\left\|\mathbb{V}_{1}\right\|$ and $r \in \mathbb{R}$. Please note that the triplet $\left(\left\|\mathbb{V}_{1}\right\|, \oplus^{\prime}, \otimes^{\prime}\right)$ is a real one-dimensional space.

The gyrometric is defined by

$$
d(\mathbf{a}, \mathbf{b})=\left\|\mathbf{a} \ominus_{E} \mathbf{b}\right\| \in\left\|\mathbb{V}_{1}\right\|,
$$

where $\mathbf{a}, \mathbf{b} \in \mathbb{V}_{1}$ and $\mathbf{a} \ominus_{E} \mathbf{b}=\mathbf{a} \oplus_{E}(-\mathbf{b})$. The gyrometric needs not be a metric. It satisfies the following [1] (p. 158).

Proposition 1. (1) For every pair $\mathbf{a}, \mathbf{b} \in \mathbb{V}_{1}, d(\mathbf{a}, \mathbf{b}) \geq 0$ The equality $d(\mathbf{a}, \mathbf{b})=0$ holds if and only if $\mathbf{a}=\mathbf{b}$.

(2) $\quad d(\mathbf{a}, \mathbf{b})=d(\mathbf{b}, \mathbf{a})$ for any $\mathbf{a}, \mathbf{b} \in \mathbb{V}_{1}$.

(3) The gyrotriangle inequality:

$$
d(\mathbf{a}, \mathbf{b}) \leq d(\mathbf{a}, \mathbf{c}) \oplus^{\prime} d(\mathbf{c}, \mathbf{b})
$$

holds for any $\mathbf{a}, \mathbf{b}, \mathbf{c}$ in $\mathbb{V}_{1}$. 
We define the metric $\delta$ on $\mathbb{V}_{1}$ induced by the gyrometric $d$. Put the map $f:\left\|\mathbb{V}_{1}\right\| \rightarrow \mathbb{R}$ by $f(x)=\tanh ^{-1}(x)$. For any $a, b \in\left\|\mathbb{V}_{1}\right\|$ and $r \in \mathbb{R}$, the map $f$ satisfies the following.

(F1) $f\left(a \oplus^{\prime} b\right)=f(a)+f(b)$

(F2) $f\left(r \otimes^{\prime} a\right)=r f(a)$

Let the map $\delta$ on $\mathbb{V}_{1}$ be given by

$$
\delta(\mathbf{a}, \mathbf{b})=f(d(\mathbf{a}, \mathbf{b}))
$$

for $\mathbf{a}, \mathbf{b} \in \mathbb{V}_{1}$.

Lemma 1. The inequality

$$
\frac{1}{\gamma_{\mathbf{a}}(1-\mathbf{a} \cdot \mathbf{b})}\|\mathbf{b}-\mathbf{a}\| \leq\left\|\mathbf{a} \ominus_{E} \mathbf{b}\right\| \leq \frac{1}{1-\mathbf{a} \cdot \mathbf{b}}\|\mathbf{b}-\mathbf{a}\|
$$

holds for every pair $\mathbf{a}, \mathbf{b} \in \mathbb{V}_{1}$.

Proof. Recall the equations (3.177) and (3.178) [1] (pp. 88-89):

$$
\begin{aligned}
& \gamma_{\mathbf{a} \oplus_{\mathbf{E}} \mathbf{b}}=\gamma_{\mathbf{a}} \gamma_{\mathbf{b}}(1+\mathbf{a} \cdot \mathbf{b}), \\
& \gamma_{\mathbf{a} \ominus_{\mathbf{E}} \mathbf{b}}=\gamma_{\mathbf{a}} \gamma_{\mathbf{b}}(1-\mathbf{a} \cdot \mathbf{b}) .
\end{aligned}
$$

By $\gamma_{\mathbf{a} \oplus_{\mathbf{E}} \mathbf{b}}=\frac{1}{\sqrt{1-\left\|\mathbf{a} \ominus_{E} \mathbf{b}\right\|}}$ we have

$$
\begin{aligned}
\left\|\mathbf{a} \ominus_{E} \mathbf{b}\right\| & =\sqrt{1-\frac{1}{\gamma_{\mathbf{a} \oplus_{\mathbf{E}} \mathbf{b}^{2}}}} \\
& =\sqrt{1-\frac{1}{\gamma_{\mathbf{a}} \gamma_{\mathbf{b}}(1-\mathbf{a} \cdot \mathbf{b})}} \\
& =\sqrt{1-\frac{\left(1-\|\mathbf{a}\|^{2}\right)\left(1-\|\mathbf{b}\|^{2}\right)}{(1-\mathbf{a} \cdot \mathbf{b})^{2}}} \\
& =\frac{\sqrt{(1-\mathbf{a} \cdot \mathbf{b})^{2}-\left(1-\|\mathbf{a}\|^{2}\right)\left(1-\|\mathbf{b}\|^{2}\right)}}{1-\mathbf{a} \cdot \mathbf{b}} \\
& =\frac{\sqrt{\|\mathbf{b}-\mathbf{a}\|^{2}-\|\mathbf{a}\|^{2}\|\mathbf{b}\|^{2}+(\mathbf{a} \cdot \mathbf{b})^{2}}}{1-\mathbf{a} \cdot \mathbf{b}} .
\end{aligned}
$$

Since $\mathbf{a} \cdot \mathbf{b} \leq\|\mathbf{a}\|\|\mathbf{b}\|$ then we have

$$
\left\|\mathbf{a} \ominus_{E} \mathbf{b}\right\| \leq \frac{1}{1-\mathbf{a} \cdot \mathbf{b}}\|\mathbf{b}-\mathbf{a}\| .
$$

Next we calculate $\left\|\mathbf{a} \ominus_{E} \mathbf{b}\right\|^{2}-\left\{\frac{1}{\gamma_{\mathbf{a}(1-\mathbf{a} \cdot \mathbf{b})}}\|\mathbf{b}-\mathbf{a}\|\right\}^{2}$.

$$
\begin{aligned}
& \frac{\|\mathbf{b}-\mathbf{a}\|^{2}-\|\mathbf{a}\|^{2}\|\mathbf{b}\|^{2}+(\mathbf{a} \cdot \mathbf{b})^{2}}{(1-\mathbf{a} \cdot \mathbf{b})^{2}}-\frac{1-\|\mathbf{a}\|^{2}}{(1-\mathbf{a} \cdot \mathbf{b})^{2}}\|\mathbf{b}-\mathbf{a}\|^{2} \\
= & \frac{1}{(1-\mathbf{a} \cdot \mathbf{b})^{2}}\left\{\|\mathbf{b}-\mathbf{a}\|^{2}-\|\mathbf{a}\|^{2}\|\mathbf{b}\|^{2}+(\mathbf{a} \cdot \mathbf{b})^{2}-\left(1-\|\mathbf{a}\|^{2}\right)\|\mathbf{b}-\mathbf{a}\|^{2}\right\} \\
= & \frac{1}{(1-\mathbf{a} \cdot \mathbf{b})^{2}}\left\{(\mathbf{a} \cdot \mathbf{b})^{2}+\|\mathbf{a}\|^{4}-2\|\mathbf{a}\|^{2}(\mathbf{a} \cdot \mathbf{b})\right\} \\
= & \frac{1}{(1-\mathbf{a} \cdot \mathbf{b})^{2}}\left(\|\mathbf{a}\|^{2}-\mathbf{a} \cdot \mathbf{b}\right)^{2} \\
\geq & 0 .
\end{aligned}
$$


Thus, we have the desired inequalities and conclude the proof.

Proposition 2. $\left(\mathbb{V}_{1}, \delta\right)$ is a complete metric space.

Proof. We first prove that $\left(\mathbb{V}_{1}, \delta\right)$ is a metric space. By (1) and (2) of Proposition 1, it is trivial that $\delta(\mathbf{a}, \mathbf{b})=0 \Leftrightarrow \mathbf{a}=\mathbf{b}$ and $\delta(\mathbf{a}, \mathbf{b})=\delta(\mathbf{b}, \mathbf{a})$ for every $\mathbf{a}, \mathbf{b} \in \mathbb{V}_{1}$. By (3) of Proposition 1 and the monotonicity of $f$, the inequality $f(d(\mathbf{a}, \mathbf{b})) \leq f(d(\mathbf{a}, \mathbf{c}) \oplus d(\mathbf{c}, \mathbf{b}))$ for every $\mathbf{a}, \mathbf{b}, \mathbf{c} \in \mathbb{V}_{1}$. By (F1) we have

$$
\delta(\mathbf{a}, \mathbf{b}) \leq \delta(\mathbf{a}, \mathbf{c})+\delta(\mathbf{c}, \mathbf{b}) .
$$

As $\mathbb{V}$ is complete, we have by Lemma 1 and the definition of $\delta(\cdot, \cdot)$ that $\left(\mathbb{V}_{1}, \delta\right)$ is complete.

We recall the gyroline and the gyrosegment [1] (Definition 6.19). Let $\mathbf{a}, \mathbf{b}$ be elements of $\mathbb{V}_{1}$. The gyroline through $\mathbf{a}$ and $\mathbf{b}$ is defined by

$$
L(\mathbf{a}, \mathbf{b})=\left\{\mathbf{a} \oplus_{E} t \otimes_{E}\left(\ominus_{E} \mathbf{a} \oplus_{E} \mathbf{b}\right): t \in \mathbb{R}\right\} .
$$

A gyrosegment with endpoints $\mathbf{a}$ and $\mathbf{b}$ is denoted by

$$
S(\mathbf{a}, \mathbf{b})=\left\{\mathbf{a} \oplus_{E} t \otimes_{E}\left(\ominus_{E} \mathbf{a} \oplus_{E} \mathbf{b}\right): 0 \leq t \leq 1\right\} .
$$

The point $\mathbf{a} \#_{t} \mathbf{b}=\mathbf{a} \oplus_{E} t \otimes_{E}\left(\ominus_{E} \mathbf{a} \oplus_{E} \mathbf{b}\right)$ is called the gyro $t$-point on a gyroline or gyrosegment. We abbreviate $\mathbf{a} \#_{\frac{1}{2}} \mathbf{b}$ by $\mathbf{a} \# \mathbf{b}$. Please note that $\mathbf{a} \# \mathbf{b}=\mathbf{b} \# \mathbf{a}$ for every pair $\mathbf{a}, \mathbf{b} \in \mathbb{V}_{1}$.

Theorem 1. For any $\mathbf{a}, \mathbf{b}, \mathbf{c} \in \mathbb{V}_{1}$ we have

$$
d(\mathbf{a} \# \mathbf{b}, \mathbf{a} \# \mathbf{c}) \leq \frac{1}{2} \otimes d(\mathbf{b}, \mathbf{c}) .
$$

Proof. To begin with the proof of the inequality (2), we show an equation related to the gyrometric and gamma factor. Recall the equations (3.197) and (6.266) [1] (pp. 93, 209):

$$
\begin{aligned}
\gamma_{\mathbf{a} \boxplus_{\mathbf{E}} \mathbf{b}} & =\frac{\left(\gamma_{\mathbf{a}}+\gamma_{\mathbf{b}}\right)^{2}}{\gamma_{\mathbf{a} \ominus \mathbf{b}}+1}-1, \\
\gamma_{\frac{1}{2} \otimes_{\mathbf{E}} \mathbf{a}} & =\sqrt{\frac{1+\gamma_{\mathbf{a}}}{2}} .
\end{aligned}
$$

Hence

$$
\gamma_{\mathbf{a} \# \mathbf{b}}=\frac{\gamma_{\mathbf{a}}+\gamma_{\mathbf{b}}}{\sqrt{2\left(\gamma_{\mathbf{a} \ominus \mathbf{b}}+1\right)}}
$$

holds. By a simple calculation, we have

$$
1-(\mathbf{a} \# \mathbf{b}) \cdot(\mathbf{a \# c})=\frac{1+\gamma_{\mathbf{a} \ominus \mathbf{b}}+\gamma_{\mathbf{b} \ominus \mathbf{c}}+\gamma_{\mathbf{c} \ominus \mathbf{a}}}{\left(\gamma_{\mathbf{a}}+\gamma_{\mathbf{b}}\right)\left(\gamma_{\mathbf{a}}+\gamma_{\mathbf{c}}\right)} .
$$

Hence we have

$$
\begin{aligned}
\gamma_{(\mathbf{a \# b}) \ominus_{\mathbf{E}}(\mathbf{a} \# \mathbf{c})} & =\gamma_{\mathbf{a} \# \mathbf{b}} \gamma_{\mathbf{a} \# \mathbf{c}}(1-(\mathbf{a} \# \mathbf{b}) \cdot(\mathbf{a} \# \mathbf{c})) \\
& =\frac{1+\gamma_{\mathbf{a} \ominus \mathbf{b}}+\gamma_{\mathbf{b} \ominus \mathbf{c}}+\gamma_{\mathbf{c} \ominus \mathbf{a}}}{2 \sqrt{\left(\gamma_{\mathbf{a} \ominus \mathbf{b}}+1\right)\left(\gamma_{\mathbf{a} \ominus \mathbf{c}}+1\right)}}
\end{aligned}
$$


and

$$
\begin{aligned}
d^{2}(\mathbf{a} \# \mathbf{b}, \mathbf{a} \# \mathbf{c}) & =1-\frac{1}{\gamma_{(\mathbf{a \# b}) \ominus_{\mathbf{E}}(\mathbf{a} \# \mathbf{c})^{2}}} \\
& =1-\frac{4\left(\gamma_{\mathbf{a} \ominus \mathbf{b}}+1\right)\left(\gamma_{\mathbf{a} \ominus \mathbf{c}}+1\right)}{\left(1+\gamma_{\mathbf{a} \ominus \mathbf{b}}+\gamma_{\mathbf{b} \ominus \mathbf{c}}+\gamma_{\mathbf{c} \ominus \mathbf{a}}\right)^{2}} .
\end{aligned}
$$

We also have

$$
\begin{aligned}
& \frac{1}{2} \otimes d(\mathbf{b}, \mathbf{c})=\tanh \left(\frac{1}{2} \tanh ^{-1} d(\mathbf{b}, \mathbf{c})\right) \\
& =\frac{\gamma_{\mathbf{b} \ominus_{\mathbf{E}} \mathbf{c}}}{1+\gamma_{\mathbf{b} \ominus_{\mathbf{E}} \mathbf{c}}} d(\mathbf{b}, \mathbf{c})
\end{aligned}
$$

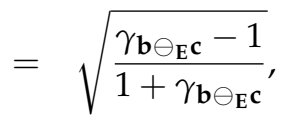

where $\gamma_{\mathbf{d}(\mathbf{b}, \mathbf{c})}=\gamma_{\mathbf{b} \ominus_{\mathbf{E}} \mathbf{c}}$. Hence we have

$$
\begin{aligned}
& \left(\frac{1}{2} \otimes d(\mathbf{b}, \mathbf{c})\right)^{2}-d^{2}(\mathbf{a} \# \mathbf{b}, \mathbf{a} \# \mathbf{c}) \\
& =\frac{\gamma_{\mathbf{b} \ominus_{\mathbf{E}} \mathbf{c}}-1}{1+\gamma_{\mathbf{b} \ominus_{\mathbf{E}} \mathbf{c}}}-\left(1-\frac{4\left(\gamma_{\mathbf{a} \ominus \mathbf{b}}+1\right)\left(\gamma_{\mathbf{a} \ominus \mathbf{c}}+1\right)}{\left(1+\gamma_{\mathbf{a} \ominus \mathbf{b}}+\gamma_{\mathbf{b} \ominus \mathbf{c}}+\gamma_{\mathbf{c} \ominus \mathbf{a}}\right)^{2}}\right) \\
& =\frac{4\left(\gamma_{\mathbf{a} \ominus \mathbf{b}}+1\right)\left(\gamma_{\mathbf{a} \ominus \mathbf{c}}+1\right)}{\left(1+\gamma_{\mathbf{a} \ominus \mathbf{b}}+\gamma_{\mathbf{b} \ominus \mathbf{c}}+\gamma_{\mathbf{c} \ominus \mathbf{a}}\right)^{2}}-\frac{2}{1+\gamma_{\mathbf{b} \ominus_{\mathbf{E}} \mathbf{c}}} \\
& =\frac{2\left\{2\left(\gamma_{\mathbf{a} \ominus \mathbf{b}}+1\right)\left(\gamma_{\mathbf{b} \ominus_{\mathbf{E}} \mathbf{c}}+1\right)\left(\gamma_{\mathbf{c} \ominus \mathbf{a}}+1\right)-\left(1+\gamma_{\mathbf{a} \ominus \mathbf{b}}+\gamma_{\mathbf{b} \ominus \mathbf{c}}+\gamma_{\mathbf{c} \ominus \mathbf{a}}\right)^{2}\right\}}{\left(1+\gamma_{\mathbf{b} \ominus_{\mathbf{E}} \mathbf{c}}\right)\left(1+\gamma_{\mathbf{a} \ominus \mathbf{b}}+\gamma_{\mathbf{b} \ominus \mathbf{c}}+\gamma_{\mathbf{c} \ominus \mathbf{a}}\right)^{2}} \\
& =\frac{2\left\{2 \gamma_{\mathbf{a} \ominus \mathbf{b}} \gamma_{\mathbf{b} \ominus \mathbf{c}} \gamma_{\mathbf{c} \ominus \mathbf{a}}-\left(\gamma_{\mathbf{a} \ominus \mathbf{b}}{ }^{2}+\gamma_{\mathbf{b} \ominus \mathbf{c}}{ }^{2}+\gamma_{\mathbf{c} \ominus \mathbf{a}^{2}}\right)+1\right\}}{\left(1+\gamma_{\mathbf{b} \ominus_{\mathbf{E}} \mathbf{c}}\right)\left(1+\gamma_{\mathbf{a} \ominus \mathbf{b}}+\gamma_{\mathbf{b} \ominus \mathbf{c}}+\gamma_{\mathbf{c} \ominus \mathbf{a}}\right)^{2}} .
\end{aligned}
$$

Let $\mathbf{A}=\ominus_{E} \mathbf{c} \oplus_{E} \mathbf{a}, \mathbf{B}=\ominus_{E} \mathbf{c} \oplus_{E} \mathbf{b}$. It is well defined by $\left\|\left(\ominus_{E} \mathbf{c} \oplus_{E} \mathbf{a}\right) \ominus_{E}\left(\ominus_{E} \mathbf{c} \oplus_{E} \mathbf{b}\right)\right\|=$ $\left\|\operatorname{gyr}\left[\ominus_{\mathbf{E}} \mathbf{c}, \mathbf{a}\right]\left(\mathbf{a} \ominus_{E} \mathbf{b}\right)\right\|=\left\|\mathbf{a} \ominus_{E} \mathbf{b}\right\|$. We calculate the numerator of (8);

$$
\begin{aligned}
& 2 \gamma_{\mathbf{a} \ominus \mathbf{b}} \gamma_{\mathbf{b} \ominus \mathbf{c}} \gamma_{\mathbf{c} \ominus \mathbf{a}}-\left(\gamma_{\mathbf{a} \ominus \mathbf{b}}{ }^{2}+\gamma_{\mathbf{b} \ominus \mathbf{c}}{ }^{2}+\gamma_{\mathbf{c} \ominus \mathbf{a}}{ }^{2}\right)+1 \\
& =2 \gamma_{\mathbf{A} \ominus_{\mathbf{E}} \mathbf{B}} \gamma_{\mathbf{A}} \gamma_{\mathbf{B}}-\left(\gamma_{\mathbf{A} \ominus_{\mathbf{E}} \mathbf{B}}{ }^{2}+\gamma_{\mathbf{A}}{ }^{2}+\gamma_{\mathbf{B}}{ }^{2}\right)+1 \\
& =2 \gamma_{\mathbf{A}}{ }^{2} \gamma_{\mathbf{B}}{ }^{2}(1-\mathbf{A} \cdot \mathbf{B})-\left(\gamma_{\mathbf{A}}{ }^{2} \gamma_{\mathbf{B}}{ }^{2}(1-\mathbf{A} \cdot \mathbf{B})^{2}+\gamma_{\mathbf{A}}{ }^{2}+\gamma_{\mathbf{B}}{ }^{2}\right)+1 \\
& =\gamma_{\mathbf{A}}^{2} \gamma_{\mathbf{B}}^{2}\left(1-(\mathbf{A} \cdot \mathbf{B})^{2}\right)-\left(\gamma_{\mathbf{A}}^{2}+\gamma_{\mathbf{B}}^{2}\right)+1 \\
& =\frac{1-(\mathbf{A} \cdot \mathbf{B})^{2}}{\left(1-\|\mathbf{A}\|^{2}\right)\left(1-\|\mathbf{B}\|^{2}\right)}-\left(\frac{1}{\left(1-\|\mathbf{A}\|^{2}\right)}+\frac{1}{\left(1-\|\mathbf{B}\|^{2}\right)}\right)+1 \\
& =\frac{\|\mathbf{A}\|^{2}\|\mathbf{B}\|^{2}-(\mathbf{A} \cdot \mathbf{B})^{2}}{\left(1-\|\mathbf{A}\|^{2}\right)\left(1-\|\mathbf{B}\|^{2}\right)} \\
& \geq 0 \text {. }
\end{aligned}
$$

We conclude a proof of Theorem 1.

By Theorem 1 and the monotonicity of $f(x)=\tanh ^{-1}(x)$, we have

$$
\delta(\mathbf{a} \# \mathbf{b}, \mathbf{a} \# \mathbf{c}) \leq \frac{1}{2} \delta(\mathbf{b}, \mathbf{c}) .
$$


By the triangle inequality, we have

$$
\begin{aligned}
\delta(\mathbf{a} \# \mathbf{b}, \mathbf{c} \# \mathbf{d}) & \leq \delta(\mathbf{a} \# \mathbf{b}, \mathbf{a} \# \mathbf{d})+\delta(\mathbf{a} \# \mathbf{d}, \mathbf{c} \# \mathbf{d}) \\
& \leq \frac{1}{2} \delta(\mathbf{b}, \mathbf{d})+\frac{1}{2} \delta(\mathbf{a}, \mathbf{c}) .
\end{aligned}
$$

Moreover, since the map $g(t)=\delta\left(\mathbf{a} \#_{t} \mathbf{b}, \mathbf{c} \#_{t} \mathbf{d}\right)$ is continuous, we infer that $g$ is convex, i.e.,

$$
\delta\left(\mathbf{a} \#_{t} \mathbf{b}, \mathbf{c} \#_{t} \mathbf{d}\right) \leq(1-t) \delta(\mathbf{a}, \mathbf{c})+t \delta(\mathbf{b}, \mathbf{d}) .
$$

Letting $\mathbf{a}=\mathbf{c}$ we have

$$
\delta\left(\mathbf{a} \#_{t} \mathbf{b}, \mathbf{a}_{t} \mathbf{c}\right) \leq t \delta(\mathbf{b}, \mathbf{c}) .
$$

\section{The Gyroconvex Set and the Gyroconvex Hull in a Gyrovector Space}

We define a gyroconvex set and a gyroconvex hull.

Definition 1. Let $A$ be a subset of $\mathbb{V}_{1}$. We say that $A$ is gyroconvex if $S(\mathbf{a}, \mathbf{b}) \subset A$ for any $\mathbf{a}, \mathbf{b} \in A$. Let $X$ be a non-empty subset of $\mathbb{V}_{1}$.

$$
\operatorname{conv}(X)=\cap\left\{C \subset \mathbb{V}_{1}: X \subset C \text { and } C \text { is gyroconvex set }\right\} .
$$

We call conv $(X)$ the gyroconvex hull of $X$.

Please note that the gyroconvex hull of a non-empty set $X \subset \mathbb{V}$ is gyroconvex.

Lemma 2. Let $a, b \in \mathbb{V}_{1}$. Then the gyrosegment $S(\mathbf{a}, \mathbf{b})$ is gyroconvex. The gyroconvex hull $\operatorname{conv}(\{\mathbf{a}, \mathbf{b}\})$ coincides with $S(\mathbf{a}, \mathbf{b})$.

Proof. Let $\mathbf{P}_{j}$ be an arbitrary point in $S(\mathbf{a}, \mathbf{b})$ for $j=1,2$. There exists $0 \leq t_{j} \leq 1$ such that

$$
\mathbf{P}_{j}=\mathbf{a} \oplus_{E} t_{j} \otimes_{E}\left(\ominus_{E} \mathbf{a} \oplus_{E} \mathbf{b}\right)
$$

for $j=1$,2. We may assume that $t_{1} \leq t_{2}$. where $0 \leq t_{1} \leq t_{2} \leq 1$. Then we have $t \in[0,1]$, $\mathbf{P}_{1} \#_{t} \mathbf{P}_{2} \in S(\mathbf{a}, \mathbf{b})$. In fact, by the Equation (6.63) in [1] (p. 167) we have

$$
\mathbf{P}_{1} \oplus_{E} t \otimes_{E}\left(\ominus_{E} \mathbf{P}_{1} \oplus_{E} \mathbf{P}_{2}\right)=\mathbf{a} \oplus_{E}\left(t_{1}+\left(-t_{1}+t_{2}\right) t\right) \otimes_{E}\left(\ominus_{E} \mathbf{a} \oplus_{E} \mathbf{b}\right) .
$$

Since $t_{1} \leq t_{1}+\left(-t_{1}+t_{2}\right) t \leq t_{2}$, we have $\mathbf{P}_{1} \#_{t} \mathbf{P}_{2} \in S(\mathbf{a}, \mathbf{b})$. Thus, $S\left(\mathbf{P}_{1}, \mathbf{P}_{2}\right) \subset S(\mathbf{a}, \mathbf{b})$ for every pair $\mathbf{P}_{1}$ and $\mathbf{P}_{2}$ in $S(\mathbf{a}, \mathbf{b})$. Thus, $S(\mathbf{a}, \mathbf{b})$ is gyroconvex.

Let $C_{0}$ be a non-empty subset of $\mathbb{V}_{1}$. We define a sequence $\left\{C_{n}\right\}$ of a non-empty subset of $\mathbb{V}_{1}$ by induction. Suppose that $C_{n-1}$ is defined. Put

$$
C_{n}=\bigcup_{\mathbf{a}, \mathbf{b} \in C_{n-1}} S(\mathbf{a}, \mathbf{b}) .
$$

Proposition 3. Let $C_{0}$ be a non-empty subset of $\mathbb{V}_{1}$. Then

$$
\operatorname{conv}\left(C_{0}\right)=\bigcup_{n=0}^{\infty} C_{n} .
$$

Proof. We prove that $\cup_{k=0}^{\infty} C_{n}$ is gyroconvex. Let $\mathbf{a}, \mathbf{b} \in \cup_{k=0}^{\infty} C_{n}$. Since $C_{k} \subset C_{k+1}$ for every $k \in \mathbb{N} \cup\{0\}$, there exists a positive integer $n_{0}$ with $\mathbf{a}, \mathbf{b} \in C_{n_{0}}$. Then by the definition of $C_{n_{0}+1}$ we have $S(\mathbf{a}, \mathbf{b}) \subset$ $C_{n_{0}+1} \subset \cup_{k=0}^{\infty} C_{n}$. Thus, $\cup_{n=0}^{\infty} C_{n}$ is a gyroconvex set. As $C_{0} \subset \cup_{k=0}^{\infty} C_{n}$, we have $\operatorname{conv}\left(C_{0}\right) \subset \cup_{n=0}^{\infty} C_{n}$. 
We prove $\cup_{n=0}^{\infty} C_{n} \subset \operatorname{conv}\left(C_{0}\right)$. For any $\mathbf{a}, \mathbf{b} \in C_{0}, S(\mathbf{a}, \mathbf{b}) \subset \operatorname{conv}\left(C_{0}\right)$. Hence $C_{1} \subset \operatorname{conv}(X)$. Similarly, assuming that $C_{n} \subset \operatorname{conv}\left(C_{0}\right)$ for any $n \in \mathbb{N} \cup\{0\}$ we have $C_{n+1} \subset \operatorname{conv}\left(C_{0}\right)$. So for arbitrary nonnegative integer $n, C_{n} \subset \operatorname{conv}\left(C_{0}\right) ; \cup_{n=0}^{\infty} C_{n} \subset \operatorname{conv}\left(C_{0}\right)$.

\section{The Gyrogeometric Mean}

Let $\phi \neq X \subset \mathbb{V}_{1}$. We define $\operatorname{diam}(X)=\sup \{\delta(\mathbf{x}, \mathbf{y}): \mathbf{x}, \mathbf{y} \in X\}$. First we prove the following.

Proposition 4. Suppose that $\mathbf{x}_{0}, \mathbf{y}_{0}, \mathbf{x}_{1}, \mathbf{y}_{1} \in \mathbb{V}_{1}$. If the inequality $\operatorname{diam}\left(\left\{\mathbf{x}_{0}, \mathbf{y}_{0}, \mathbf{x}_{1}, \mathbf{y}_{1}\right\}\right) \leq M$ holds for a positive real number $M$, then the inequality $\delta(\mathbf{x}, \mathbf{y}) \leq M$ holds for arbitrary points $\mathbf{x} \in S\left(\mathbf{x}_{0}, \mathbf{x}_{1}\right)$ and $\mathbf{y} \in S\left(\mathbf{y}_{0}, \mathbf{y}_{1}\right)$.

Proof. Put $\mathbf{x}=\mathbf{x}_{0} \#_{t} \mathbf{x}_{1}$ and $\mathbf{y}=\mathbf{y}_{0} \#_{s} \mathbf{y}_{1}$, where $0 \leq s \leq t \leq 1$. We have

$$
\begin{aligned}
& d\left(\mathbf{y}_{0} \#_{t} \mathbf{x}_{1}, \mathbf{y}_{0} \#_{s} \mathbf{x}_{1}\right) \\
= & \left\|\left\{\mathbf{y}_{0} \oplus_{E} t \otimes_{E}\left(\ominus_{E} \mathbf{y}_{0} \oplus_{E} \mathbf{x}_{1}\right)\right\} \ominus_{E}\left\{\mathbf{y}_{0} \oplus_{E} s \otimes_{E}\left(\ominus_{E} \mathbf{y}_{0} \oplus_{E} \mathbf{x}_{1}\right)\right\}\right\| \\
= & \left\|\operatorname{gyr}\left[\ominus_{E} \mathbf{y}_{0}, t \otimes_{E}\left(\ominus_{E} \mathbf{y}_{\mathbf{0}} \oplus_{E} \mathbf{x}_{\mathbf{1}}\right)\right]\left\{t \otimes_{E}\left(\ominus_{E} \mathbf{y}_{0} \oplus_{E} \mathbf{x}_{1}\right) \ominus_{E} s \otimes_{E}\left(\ominus_{E} \mathbf{y}_{0} \oplus_{E} \mathbf{x}_{1}\right)\right\}\right\| \\
= & (t-s) \otimes\left\|\ominus_{E} \mathbf{y}_{0} \oplus_{E} \mathbf{x}_{1}\right\| .
\end{aligned}
$$

Applying this equality, we have by (11) and (12) that

$$
\begin{aligned}
\delta(\mathbf{x}, \mathbf{y}) & \leq \delta\left(\mathbf{x}, \mathbf{y}_{0} \#_{t} \mathbf{x}_{1}\right)+\delta\left(\mathbf{y}_{0} \#_{t} \mathbf{x}_{1}, \mathbf{y}_{0} \#_{s} \mathbf{x}_{1}\right)+\delta\left(\mathbf{y}_{0} \#_{s} \mathbf{x}_{1}, \mathbf{y}\right) \\
& =\delta\left(\mathbf{x}_{0} \#_{t} \mathbf{x}_{1}, \mathbf{y}_{0} \#_{t} \mathbf{x}_{1}\right)+(t-s) \delta\left(\mathbf{y}_{0}, \mathbf{x}_{1}\right)+\delta\left(\mathbf{y}_{0} \#_{s} \mathbf{x}_{1}, \mathbf{y}_{0} \#_{s} \mathbf{y}_{1}\right) \\
& \leq(1-t) \delta\left(\mathbf{x}_{0}, \mathbf{y}_{0}\right)+(t-s) \delta\left(\mathbf{y}_{0}, \mathbf{x}_{1}\right)+s \delta\left(\mathbf{x}_{1}, \mathbf{y}_{1}\right) \\
& \leq(1-t) M+(t-s) M+s M=M
\end{aligned}
$$

Lemma 3. Let $X \subset \mathbb{V}$ be a non-empty set. Then

$$
\operatorname{diam}(X)=\operatorname{diam}(\operatorname{conv}(X))=\operatorname{diam}(\overline{\operatorname{conv}(X)}) .
$$

Proof. First we prove $\operatorname{diam}(X)=\operatorname{diam}(\operatorname{conv}(X))$. By Proposition $3, \operatorname{conv}(X)=\bigcup_{n=0}^{\infty} C_{n}$ where $C_{0}=X$. For any positive integer $k$ let $\mathbf{x}, \mathbf{y}$ be arbitrary points in $C_{k}$. Then there exist $\mathbf{x}_{0}, \mathbf{x}_{1}, \mathbf{y}_{0}, \mathbf{y}_{1} \in C_{k-1}$ such that $\mathbf{x} \in S\left(\mathbf{x}_{0}, \mathbf{x}_{1}\right), \mathbf{y} \in S\left(\mathbf{y}_{0}, \mathbf{y}_{1}\right)$. Put $M=\operatorname{diam}\left(C_{k-1}\right)$ then by Proposition 4.1 we have

$$
\delta(\mathbf{x}, \mathbf{y}) \leq M=\operatorname{diam}\left(C_{k-1}\right),
$$

whence

$$
\operatorname{diam}\left(C_{k}\right) \leq \operatorname{diam}\left(C_{k-1}\right) .
$$

Thus, for arbitrary $n \in \mathbb{N}, \operatorname{diam}\left(C_{n}\right) \leq \operatorname{diam}\left(C_{0}\right)=\operatorname{diam}(X)$. It follows that for any $\mathbf{x}, \mathbf{y} \in \operatorname{conv}(X)=$ $\cup_{n=0}^{\infty} C_{n}$, we have

$$
\delta(\mathbf{x}, \mathbf{y}) \leq \operatorname{diam}(X)
$$

Therefore

$$
\operatorname{diam}(\operatorname{conv}(X)) \leq \operatorname{diam}(X) .
$$

The converse inequality is trivial, hence we have $\operatorname{diam}(\operatorname{conv}(X))=\operatorname{diam}(X)$.

Next we prove $\operatorname{diam}(\operatorname{conv}(X))=\operatorname{diam}(\overline{\operatorname{conv}(X)})$. For any pair $\mathbf{x}, \mathbf{y} \in \overline{\operatorname{conv}(X)}$, there exist sequences $\left\{\mathbf{x}_{n}\right\}$ and $\left\{\mathbf{y}_{n}\right\}$ in conv $(X)$ such that $\mathbf{x}_{n}, \mathbf{y}_{n}$ converge to $\mathbf{x}, \mathbf{y}$ respectively. Letting $n \rightarrow \infty$ for $\delta\left(\mathbf{x}_{n}, \mathbf{y}_{n}\right) \leq \operatorname{diam}(\operatorname{conv}(X))$, we have $\delta(\mathbf{x}, \mathbf{y}) \leq \operatorname{diam}(X) . \operatorname{Thus,} \operatorname{diam}(\overline{\operatorname{conv}(X)}) \leq \operatorname{diam}(\operatorname{conv}(X))$. The converse inequality is trivial, hence $\operatorname{diam}(\operatorname{conv}(X))=\operatorname{diam}(\overline{\operatorname{conv}(X)})$ holds. 
Lemma 4. Suppose that $K$ is a gyroconvex subset of $\mathbb{V}$. Then the closure $\bar{K}$ of $K$ is gyroconvex.

Proof. For any $\mathbf{x}, \mathbf{y} \in \bar{K}$, there exist $\left\{\mathbf{x}_{n}\right\},\left\{\mathbf{y}_{n}\right\} \subset K$ such that $\mathbf{x}_{n}, \mathbf{y}_{n}$ converge to $\mathbf{x}, \mathbf{y}$ respectively. We show $\mathbf{x}_{n} \#_{t} \mathbf{y}_{n}$ converges to $\mathbf{x}_{t} \mathbf{y}$ for arbitrary $0 \leq t \leq 1$. By (11) we have

$$
\delta\left(\mathbf{x}_{n} \#_{t} \mathbf{y}_{n}, \mathbf{x}_{t} \mathbf{y}\right) \leq(1-t) \delta\left(\mathbf{x}_{n}, \mathbf{x}\right)+t \delta\left(\mathbf{y}_{n}, \mathbf{y}\right)
$$

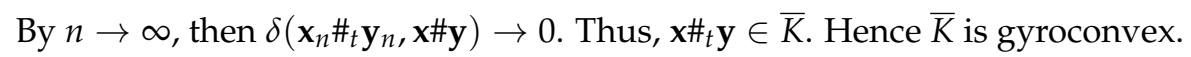

Let $n$ be a positive integer. Let $X_{n}$ be the set of all subsets of $\mathbb{V}_{1}$ whose number of elements is exactly $n$. We define, by induction, the sequence $\left\{G_{n}\right\}_{n=2}^{\infty}$ of the maps $G_{n}: X_{n} \rightarrow \mathbb{V}_{1}$ which satisfy the following two conditions $\left(p_{n}\right)$ and $\left(q_{n}\right)$;

$\left(p_{n}\right) \quad G_{n}(\Delta) \in \overline{\operatorname{conv}(\Delta)}$ for every $\Delta \in X_{n}$,

$\left(q_{n}\right) \quad \delta\left(G_{n}(\Delta), G_{n}\left(\Delta^{\prime}\right)\right) \leq \frac{1}{n} \sum_{i=1}^{n} \delta\left(\mathbf{a}_{i}, \mathbf{a}_{i}^{\prime}\right)$ for every pair $\Delta=\left\{\mathbf{a}_{1}, \ldots, \mathbf{a}_{n}\right\}$ and $\Delta^{\prime}=\left\{\mathbf{a}_{1}^{\prime}, \ldots, \mathbf{a}_{n}^{\prime}\right\}$ in $X_{n}$.

First, put $G_{2}(\{\mathbf{a}, \mathbf{b}\})=\mathbf{a} \# \mathbf{b}$ for $\{\mathbf{a}, \mathbf{b}\} \in X_{2}$. As $\operatorname{conv}(\{\mathbf{a}, \mathbf{b}\})=S(\mathbf{a}, \mathbf{b})$ by Lemma 2 we obtain that $G_{2}(\{\mathbf{a}, \mathbf{b}\}) \in \operatorname{conv}(\{\mathbf{a}, \mathbf{b}\}) ;\left(p_{2}\right)$ holds. Let $\Delta=\{\mathbf{a}, \mathbf{b}\}, \Delta^{\prime}=\{\mathbf{c}, \mathbf{d}\} \in X_{2}$. Then by (10) we have

$$
\delta\left(G_{2}(\Delta), G_{2}\left(\Delta^{\prime}\right)\right)=\delta(\mathbf{a} \# \mathbf{b}, \mathbf{c \# d}) \leq \frac{1}{2}\{\delta(\mathbf{a}, \mathbf{c})+\delta(\mathbf{b}, \mathbf{d})\}
$$

which is $\left(q_{2}\right)$.

Suppose now that the map $G_{k}: X_{k} \rightarrow \mathbb{V}_{1}$ which satisfies $\left(p_{k}\right)$ and $\left(q_{k}\right)$ is defined. We will define $G_{k+1}: X_{k+1} \rightarrow \mathbb{V}_{1}$ which satisfies $\left(p_{k+1}\right)$ and $\left(q_{k+1}\right)$. Let $\Delta^{0}=\left\{\mathbf{a}_{1}^{0}, \ldots, \mathbf{a}_{k+1}^{0}\right\} \in X_{k+1}$. For a positive integer $m$ we define $\Delta^{m} \in X_{k+1}$ which satisfies that $\Delta^{m} \subset \overline{\operatorname{conv}\left(\Delta^{m-1}\right)}$ by induction on $m$. For every $1 \leq i \leq k+1$, put

$$
\mathbf{a}_{i}^{1}=G_{k}\left(\left\{\mathbf{a}_{1}^{0}, \ldots, \mathbf{a}_{i-1}^{0}, \mathbf{a}_{i+1}^{0}, \ldots, \mathbf{a}_{k+1}^{0}\right\}\right) .
$$

Please note that $\mathbf{a}_{i}^{1}$ is well defined since $\left\{\mathbf{a}_{1}^{0}, \ldots, \mathbf{a}_{i-1}^{0}, \mathbf{a}_{i+1}^{0}, \ldots, \mathbf{a}_{k+1}^{0}\right\} \in X_{k}$ and we have assumed that the map $G_{k}$ is defined. By the condition $\left(p_{k}\right)$ we have that

$$
\mathbf{a}_{i}^{1} \in \overline{\operatorname{conv}\left(\left\{\mathbf{a}_{1}^{0}, \ldots, \mathbf{a}_{i-1}^{0}, \mathbf{a}_{i+1}^{0}, \ldots, \mathbf{a}_{k+1}^{0}\right\}\right)}
$$

for every $1 \leq i \leq k+1$. Put $\Delta^{1}=\left\{\mathbf{a}_{1}^{1}, \ldots, \mathbf{a}_{k+1}^{1}\right\}$. Then $\Delta^{1} \in X_{k+1}$ and $\Delta^{1} \subset \overline{\operatorname{conv}\left(\Delta^{0}\right)}$ since $\left\{\mathbf{a}_{1}^{0}, \ldots, \mathbf{a}_{i-1}^{0}, \mathbf{a}_{i+1}^{0}, \ldots, \mathbf{a}_{k+1}^{0}\right\} \subset \Delta^{0}$ for every $1 \leq i \leq k+1$. Suppose that $\Delta^{l}=\left\{\mathbf{a}_{1}^{l}, \ldots, \mathbf{a}_{k+1}^{l}\right\} \in X_{k+1}$ such that $\Delta^{l} \subset \overline{\operatorname{conv}\left(\Delta^{l-1}\right)}$ is defined. For every $1 \leq i \leq k+1$, put

$$
\mathbf{a}_{i}^{l+1}=G_{k}\left(\left\{\mathbf{a}_{1}^{l}, \ldots, \mathbf{a}_{i-1}^{l}, \mathbf{a}_{i+1}^{l}, \ldots, \mathbf{a}_{k+1}^{l}\right\}\right) .
$$

As in the same way as the above, $\mathbf{a}_{i}^{l+1}$ is well defined for $1 \leq i \leq k+1$, and $\Delta^{l+1}=\left\{\mathbf{a}_{1}^{l+1}, \ldots, \mathbf{a}_{k+1}^{l+1}\right\} \in$ $X_{k+1}$ satisfies that $\Delta^{l+1} \subset \overline{\operatorname{conv}\left(\Delta^{l}\right)}$. Hence, by induction, we have defined a sequence $\left\{\Delta^{m}\right\}_{m=1}^{\infty} \subset$ $X_{k+1}$ such that $\Delta^{m} \subset \overline{\operatorname{conv}\left(\Delta^{m-1}\right)}$. Applying $\left(q_{k}\right)$ for $\Delta=\left\{\mathbf{a}_{1}^{m}, \ldots, \mathbf{a}_{i-1}^{m}, \mathbf{a}_{i+1}^{m}, \ldots, \mathbf{a}_{k+1}^{m}\right\}$ and $\Delta^{\prime}=$ $\left\{\mathbf{a}_{1}^{m}, \ldots, \mathbf{a}_{j-1}^{m}, \mathbf{a}_{j+1}^{m}, \ldots, \mathbf{a}_{k+1}^{m}\right\}$, we infer that

$$
\begin{aligned}
\begin{aligned}
& \delta\left(\mathbf{a}_{i}^{m+1}, \mathbf{a}_{j}^{m+1}\right)=\delta(\left.G_{k}(\Delta), G_{k}\left(\Delta^{\prime}\right)\right) \\
&=\delta\left(G_{k}(\right.\left.\left.\left\{\mathbf{a}_{1}^{m}, \ldots, \mathbf{a}_{i-1}^{m}, \mathbf{a}_{i+1}^{m}, \ldots, \mathbf{a}_{k+1}^{m}\right\}\right), G_{k}\left(\left\{\mathbf{a}_{1}^{m}, \ldots, \mathbf{a}_{j-1}^{m}, \mathbf{a}_{j+1}^{m}, \ldots, \mathbf{a}_{k+1}^{m}\right\}\right)\right) \\
&= \delta\left(G_{k}\left(\left\{\mathbf{a}_{1}^{m}, \ldots, \mathbf{a}_{i-1}^{m}, \mathbf{a}_{i+1}^{m}, \ldots, \mathbf{a}_{j-1}^{m}, \mathbf{a}_{j+1}^{m}, \ldots, \mathbf{a}_{k+1}^{m}, \mathbf{a}_{j}^{m}\right\}\right),\right. \\
&\left.G_{k}\left(\left\{\mathbf{a}_{1}^{m}, \ldots, \mathbf{a}_{i-1}^{m}, \mathbf{a}_{i+1}^{m}, \ldots, \mathbf{a}_{j-1}^{m}, \mathbf{a}_{j+1}^{m}, \ldots, \mathbf{a}_{k+1}^{m}, \mathbf{a}_{i}^{m}\right\}\right)\right) \\
& \quad \leq \frac{1}{k} \delta\left(\mathbf{a}_{j}^{m}, \mathbf{a}_{i}^{m}\right)=\frac{1}{k} \delta\left(\mathbf{a}_{i}^{m}, \mathbf{a}_{j}^{m}\right)
\end{aligned}
\end{aligned}
$$


Then by Lemma 3 we obtain

$$
\operatorname{diam}\left(\overline{\operatorname{conv}\left(\Delta^{m+1}\right)}\right) \leq \frac{1}{k} \operatorname{diam}\left(\overline{\operatorname{conv}\left(\Delta^{m}\right)}\right)
$$

for every positive integer $m$. By Cantor's intersection theorem there exists a unique $M_{\infty} \in \mathbb{V}_{1}$ with

$$
\left\{M_{\infty}\right\}=\cap_{m} \overline{\operatorname{conv}\left(\Delta^{m}\right)} .
$$

As $\left.a_{i}^{m} \in \overline{\operatorname{conv}\left(\Delta^{m-1}\right.}\right)$ for every $1 \leq i \leq k+1$, we infer that $\lim _{m \rightarrow \infty} \mathbf{a}_{i}^{m}=M_{\infty}$ for every $1 \leq i \leq k+1$. Put $G_{k+1}\left(\Delta^{0}\right)=M_{\infty}$. Then the map $G_{k+1}: X_{k+1} \rightarrow \mathbb{V}_{1}$ is well defined, and $G_{k+1}\left(\Delta^{0}\right)=M_{\infty} \in$ $\overline{\operatorname{conv}\left(\Delta^{0}\right)} ; G_{k+1}$ satisfies the condition $\left(p_{n}\right)$.

Next we prove that the map $G_{k+1}$ satisfies the condition $\left(q_{k+1}\right)$;

$$
\delta\left(G_{k+1}(\Delta), G_{k+1}\left(\Delta^{\prime}\right)\right) \leq \frac{1}{k+1}\left(\sum_{j=1}^{k+1} \delta\left(\mathbf{a}_{j}, \mathbf{a}_{j}^{\prime}\right)\right)
$$

where $\Delta=\left\{\mathbf{a}_{1}^{0}, \ldots, \mathbf{a}_{k+1}^{0}\right\}, \Delta^{\prime}=\left\{\mathbf{a}_{1}^{\prime 0}, \ldots, \mathbf{a}_{k+1}^{\prime 0}\right\} \in X_{k+1}$. Let $m$ be a positive integer. We define $\mathbf{a}_{i}^{m}$ and $\mathbf{a}_{i}^{\prime m}$ for every $1 \leq i \leq k+1$ as in the same way as before. As $\left(q_{k}\right)$ holds for $G_{k}$, we have

$$
\begin{aligned}
& \delta\left(\mathbf{a}_{i}^{m}, \mathbf{a}_{i}^{\prime m}\right) \\
&=\delta\left(G_{k}\left(\left\{\mathbf{a}_{1}^{m-1}, \mathbf{a}_{2}^{m-1}, \ldots, \mathbf{a}_{i-1}^{m-1}, \mathbf{a}_{i+1}^{m-1}, \ldots, \mathbf{a}_{k+1}^{m-1}\right\}\right),\right. \\
&\left.G_{k}\left(\left\{\mathbf{a}_{1}^{\prime m-1}, \mathbf{a}_{2}^{\prime m-1}, \ldots, \mathbf{a}_{i-1}^{\prime m-1}, \mathbf{a}^{\prime m-1}, \ldots, \mathbf{a}^{\prime m-1}{ }_{k+1}^{m-1}\right\}\right)\right) \\
& \quad \leq \frac{1}{k}\left(\sum_{j=1, j \neq i}^{k+1} \delta\left(\mathbf{a}_{j}^{m-1}, \mathbf{a}_{j}^{\prime m-1}\right)\right) .
\end{aligned}
$$

By summing up the above inequalities with respect to $1 \leq i \leq k+1$ we have

$$
\sum_{i=1}^{k+1} \delta\left(\mathbf{a}_{i}^{m}, \mathbf{a}_{i}^{\prime m}\right) \leq \sum_{i=1}^{k+1}\left(\frac{1}{k}\left(\sum_{j=1, j \neq i}^{k+1} \delta\left(\mathbf{a}_{j}^{m-1}, \mathbf{a}_{j}^{\prime m-1}\right)\right)\right)=\sum_{j=1}^{k+1} \delta\left(\mathbf{a}_{j}^{m-1}, \mathbf{a}_{j}^{\prime m-1}\right) .
$$

for every positive integer $m$. Thus, we have

$$
\sum_{i=1}^{k+1} \delta\left(\mathbf{a}_{i}^{m}, \mathbf{a}_{i}^{\prime m}\right) \leq \sum_{j=1}^{k+1} \delta\left(\mathbf{a}_{j}, \mathbf{a}_{j}^{\prime}\right)
$$

Letting $m \rightarrow \infty$, since $\lim _{m \rightarrow \infty} \mathbf{a}_{i}^{m}=G_{k+1}(\Delta), \lim _{m \rightarrow \infty} \mathbf{a}_{i}^{\prime m}=G_{k+1}\left(\Delta^{\prime}\right)$, we have

$$
\sum_{i=1}^{k+1} \delta\left(G_{k+1}(\Delta), G_{k+1}\left(\Delta^{\prime}\right)\right) \leq \sum_{j=1}^{k+1} \delta\left(\mathbf{a}_{j}, \mathbf{a}_{j}^{\prime}\right),
$$

hence

$$
\left.\delta\left(G_{k+1}(\Delta)\right), G_{k+1}\left(\Delta^{\prime}\right)\right) \leq \frac{1}{k+1}\left(\sum_{j=1}^{k+1} \delta\left(\mathbf{a}_{j}, \mathbf{a}_{j}^{\prime}\right)\right) .
$$

So, the condition $\left(q_{k+1}\right)$ holds for the map $G_{k+1}$. We conclude that the map $G_{n}: X_{n} \rightarrow \mathbb{V}_{1}$ which satisfies the conditions $\left(p_{n}\right)$ and $\left(q_{n}\right)$ are defined by induction.

By applying the maps $G_{n}$ we define the gyrogeometric mean of $n$ elements in $\mathbb{V}_{1}$.

Definition 2. Let $\Delta=\left\{\mathbf{a}_{1}, \ldots, \mathbf{a}_{n}\right\} \subset \mathbb{V}_{1}$. We call that $G_{n}(\Delta)$ the gyrogeometric mean of $\Delta$. 
Due to the definition, the gyrogeometric mean of $\{\mathbf{a}, \mathbf{b}\} \subset \mathbb{V}_{1}$ is $\mathbf{a} \# \mathbf{b}$. The gyrocentroid $\mathbb{C}_{\mathbf{a b c}}^{m}$ is defined by applying the internal division points on the usual lines which makes the inconvenience such as $\mathbb{C}_{\mathrm{abc}}^{m}=\frac{\gamma_{\mathbf{c}}}{\gamma_{\mathbf{c}}+2} \mathbf{c} \neq \frac{1}{3} \otimes_{E} \mathbf{c}$ for $\mathbf{a}=\mathbf{b}=0$. The gyrogeometric mean is defined by applying the gyrolines and it resolve the inconvenience.

\section{Properties of the Gyrogeometric Mean}

The gyrogeometric mean satisfies certain desirable properties one would expect for means in general. For example, the permutation invariance and the left-translation invariance would be expected properties. It is trivial that the gyrogeometric mean is permutation-invariant. We prove that the gyrogeometric mean is left-translation invariant.

Recall that $X_{n}$ is the set of all $n$-points subset of $\mathbb{V}_{1}$ for a positive integer $n$.

Proposition 5. Let $\mathbf{x} \in \mathbb{V}_{1}$ and $D_{n}=\left\{\mathbf{a}_{1}, \mathbf{a}_{2}, \cdots, \mathbf{a}_{n}\right\} \in X_{n}$. Put $\mathbf{x} \oplus_{E} D_{n}=\left\{\mathbf{x} \oplus_{E} \mathbf{a}_{1}, \mathbf{x} \oplus_{E} \mathbf{a}_{2}, \cdots, \mathbf{x} \oplus_{E}\right.$ $\left.\mathbf{a}_{n}\right\}$. Then the following holds:

$$
G_{n}\left(\mathbf{x} \oplus_{E} D_{n}\right)=\mathbf{x} \oplus_{E} G_{n}\left(D_{n}\right) .
$$

Proof. We prove the equality (13) by induction on $n$. For $n=2, G_{2}\left(\left\{\mathbf{a}_{1}, \mathbf{a}_{2}\right\}\right)=\mathbf{P}_{\mathbf{a}_{1} \mathbf{a}_{2}}^{m}$. By Theorem 6.37 in [1] (p. 175) we have

$$
G_{2}\left(\left\{\mathbf{x} \oplus_{E} \mathbf{a}_{1}, \mathbf{x} \oplus_{E} \mathbf{a}_{2}\right\}\right)=\mathbf{x} \oplus_{E} G_{2}\left(\left\{\mathbf{a}_{1}, \mathbf{a}_{2}\right\}\right) .
$$

Assume that (13) holds for $n=k$. Let $D_{k+1}^{m}=\left\{\mathbf{a}_{1}^{m}, \mathbf{a}_{2}^{m}, \cdots, \mathbf{a}_{k+1}^{m}\right\}$ where $\mathbf{a}_{i}^{m}=$ $G_{k}\left(\left\{\mathbf{a}_{1}^{m-1}, \mathbf{a}_{2}^{m-1}, \cdots, \mathbf{a}_{i-1}^{m-1}, \mathbf{a}_{i+1}^{m-1}, \cdots, \mathbf{a}_{k+1}\right\}\right)$ for $1 \leq i \leq k+1$. By the assumption we have

$$
\begin{array}{r}
G_{k}\left(\left\{\mathbf{x} \oplus_{E} \mathbf{a}_{1}^{m-1}, \mathbf{x} \oplus_{E} \mathbf{a}_{2}^{m-1}, \cdots, \mathbf{x} \oplus_{E} \mathbf{a}_{i-1}^{m-1}, \mathbf{x} \oplus_{E} \mathbf{a}_{i+1}^{m-1}, \ldots, \mathbf{x} \oplus_{E} \mathbf{a}_{k+1}^{m-1}\right\}\right) \\
=\mathbf{x} \oplus_{E} G_{k}\left(\left\{\mathbf{a}_{1}^{m-1}, \mathbf{a}_{2}^{m-1}, \cdots, \mathbf{a}_{i-1}^{m-1}, \mathbf{a}_{i+1}^{m-1}, \cdots, \mathbf{a}_{k+1}^{m-1}\right\}\right) \\
=\mathbf{x} \oplus_{E} \mathbf{a}_{i}^{m}
\end{array}
$$

for every $1 \leq i \leq k+1$. Then for $\mathbf{x} \oplus_{E} D_{k+1}=\left\{\mathbf{x} \oplus_{E} \mathbf{a}_{1}, \mathbf{x} \oplus_{E} \mathbf{a}_{2}, \cdots, \mathbf{x} \oplus_{E} \mathbf{a}_{k+1}\right\}$ we have

$$
\begin{array}{r}
\mathbf{x} \oplus_{E} D_{k+1}^{m} \\
\qquad\left\{G_{k}\left(\left\{\mathbf{x} \oplus_{E} \mathbf{a}_{1}^{m-1}, \cdots, \mathbf{x} \oplus_{E} \mathbf{a}_{i-1}^{m-1}, \mathbf{x} \oplus_{E} \mathbf{a}_{i+1}^{m-1}, \cdots, \mathbf{x} \oplus_{E} \mathbf{a}_{k+1}^{m-1}\right\}\right): 1 \leq i \leq k+1\right\} \\
=\left\{\mathbf{x} \oplus_{E} \mathbf{a}_{i}^{m}: i=1,2, \cdots, k+1\right\} .
\end{array}
$$

We prove that $\mathbf{x} \oplus_{E} D_{k+1}^{m} \rightarrow\left\{\mathbf{x} \oplus_{E} G_{k+1}\left(D_{k+1}\right)\right\}$ as $m \rightarrow \infty$. By a simple calculation we have

$$
d\left(\mathbf{x} \oplus_{E} \mathbf{a}_{i}^{m}, \mathbf{x} \oplus_{E} G_{k+1}\left(D_{k+1}\right)\right)
$$

$$
=\left\|\operatorname{gyr}\left[\mathbf{x}, \mathbf{a}_{\mathbf{i}}^{\mathbf{m}}\right]\left(\mathbf{a}_{i}^{m} \ominus_{E} G\left(D_{k+1}\right)\right)\right\|=\left\|\mathbf{a}_{i}^{m} \ominus_{E} G\left(D_{k+1}\right)\right\| \rightarrow 0
$$

as $m \rightarrow \infty$. Hence we have $\delta\left(\mathbf{x} \oplus_{E} \mathbf{a}_{i}^{m}, \mathbf{x} \oplus_{E} G_{k+1}\left(D_{k+1}\right)\right) \rightarrow 0$ as $m \rightarrow \infty$. Thus, $\mathbf{x} \oplus_{E} \mathbf{a}_{i}^{m} \rightarrow \mathbf{x} \oplus_{E}$ $G\left(D_{k+1}\right)$ as $m \rightarrow \infty$ for $1 \leq i \leq k+1$. We conclude that $G_{n}\left(\mathbf{x} \oplus_{E} D_{n}\right)=\mathbf{x} \oplus_{E} G_{n}\left(D_{n}\right)$.

For $\mathbf{a}=\mathbf{b}=\mathbf{0}$ and $\mathbf{c}$ in $\mathbb{V}_{1}, G_{3}(\{\mathbf{a}, \mathbf{b}, \mathbf{c}\})=\frac{1}{3} \otimes_{E} \mathbf{c}$. More generally, the gyrogeometric mean satisfies the following.

Proposition 6. Let $\Delta=\left\{t_{1} \otimes_{E} \mathbf{a}, t_{2} \otimes_{E} \mathbf{a}, \cdots, t_{n} \otimes_{E} \mathbf{a}\right\} \subset \mathbb{V}_{1}$.

$$
G_{n}(\Delta)=\frac{t_{1}+t_{2}+\cdots+t_{n}}{n} \otimes_{E} \mathbf{a}
$$


In the case of $n=2$, it is proved by the following calculation.

$$
\begin{aligned}
& G_{2}\left(t_{1} \otimes_{E} \mathbf{a}, t_{2} \otimes_{E} \mathbf{a}\right) \\
= & t_{1} \otimes_{E} \mathbf{a} \# t_{2} \otimes_{E} \mathbf{a} \\
= & \frac{1}{2} \otimes_{E}\left\{\left(t_{1} \otimes_{E} \mathbf{a}\right) \boxplus_{E}\left(t_{2} \otimes_{E} \mathbf{a}\right)\right\} \\
= & \frac{1}{2} \otimes_{E}\left\{\left(t_{1} \otimes_{E} \mathbf{a}\right) \oplus_{E} \operatorname{gyr}\left[\mathbf{t}_{1} \otimes_{\mathbf{E}} \mathbf{a}, \ominus_{\mathbf{E}} \mathbf{t}_{2} \otimes_{\mathbf{E}} \mathbf{a}\right]\left(t_{2} \otimes_{E} \mathbf{a}\right)\right\} \\
= & \frac{1}{2} \otimes_{E}\left\{\left(t_{1} \otimes_{E} \mathbf{a}\right) \oplus_{E}\left(t_{2} \otimes_{E} \mathbf{a}\right)\right\} \\
= & \frac{1}{2} \otimes_{E}\left\{\left(t_{1}+t_{2}\right) \otimes \mathbf{a}\right\} \\
= & \frac{t_{1}+t_{2}}{2} \otimes_{E} \mathbf{a} .
\end{aligned}
$$

Proposition 6 is proved by induction on $n$.

In Section $6 \mathbb{V}_{s}=\{\mathbf{v} \in \mathbb{V}:\|\mathbf{v}\|<s\}$ with appropriate operation is a gyrocommutative gyrogroup, which is also called the Einstein gyrogroup. The gyrogeometric mean is defined for $\mathbb{V}_{s}$ similarly. If $s \rightarrow \infty$ or $\mathbf{v} \in \mathbb{V}_{s}$ such that $\|\mathbf{v}\|$ is small enough, $\gamma_{\mathbf{v}} \rightarrow 1$. So, in the case,

$$
G_{n}\left(\left\{\mathbf{a}_{1}, \mathbf{a}_{2}, \cdots, \mathbf{a}_{n}\right\}\right) \rightarrow \frac{\mathbf{a}_{1}+\mathbf{a}_{2}+\cdots+\mathbf{a}_{n}}{n}
$$

is hold. It is simply proved by induction.

\section{Proof that $\left(\mathbb{V}_{s}, \oplus_{E}\right)$ Is a Gyrocommutative Gyrogroup}

A magma $(G, \oplus)$ is a non-empty set $G$ with a binary operation $\oplus$. A magma $(G, \oplus)$ is a gyrogroup if its binary operation $\oplus$ satisfies the following axioms (G1) through (G5):

(G1) There exists a left identity $\mathbf{0}$ in $G$ such that

$$
\mathbf{0} \oplus \mathbf{a}=\mathbf{a}
$$

for all $\mathbf{a} \in G$.

(G2) For each $\mathbf{a} \in G$ there exists a left inverse $\ominus \mathbf{a} \in G$ such that

$$
\ominus \mathbf{a} \oplus \mathbf{a}=\mathbf{0} .
$$

(G3) For any $\mathbf{a}, \mathbf{b}, \mathbf{c} \in G$ there exists a unique element $\operatorname{gyr}[\mathbf{a}, \mathbf{b}] \mathbf{c} \in G$ such that the binary operation obeys the left gyroassociative law

$$
\mathbf{a} \oplus(\mathbf{b} \oplus \mathbf{c})=(\mathbf{a} \oplus \mathbf{b}) \oplus \operatorname{gyr}[\mathbf{a}, \mathbf{b}] \mathbf{c} .
$$

(G4) The map $\operatorname{gyr}[\mathbf{a}, \mathbf{b}]: G \mapsto G$ given by $\mathbf{c} \mapsto \operatorname{gyr}[\mathbf{a}, \mathbf{b}] \mathbf{c}$ is an automorphism of the magma $(G, \oplus)$. It is called a gyroautomorphism. gyr $[\mathbf{a}, \mathbf{b}]$ generated by $\mathbf{a}, \mathbf{b} \in G$ is called a gyration.

(G5) The gyroautomorphism gyr $[\mathbf{a}, \mathbf{b}]$ generated by any $\mathbf{a}, \mathbf{b} \in G$ satisfies the left loop property:

$$
\operatorname{gyr}[\mathbf{a}, \mathbf{b}]=\operatorname{gyr}[\mathbf{a} \oplus \mathbf{b}, \mathbf{b}] .
$$

The gyrogroup $(G, \oplus)$ is called gyrocommutative if the following (G6) holds for every pair $\mathbf{a}, \mathbf{b} \in G$

$$
\text { (G6) } \mathbf{a} \oplus \mathbf{b}=\operatorname{gyr}[\mathbf{a}, \mathbf{b}](\mathbf{b} \oplus \mathbf{a}) \text {. }
$$

We prove that the Einstein gyrogroup $\left(\mathbb{V}_{S}, \oplus_{E}\right)$ is in fact a gyrocommutative gyrogroup only by simple calculations. Proof of (G1) and (G2) are simple and omitted. 
We prove (G3). We prove that $\mathbf{u} \oplus_{E}\left(\mathbf{v} \oplus_{E} \mathbf{w}\right)=\left(\mathbf{u} \oplus_{E} \mathbf{v}\right) \oplus_{E} \operatorname{gyr}[\mathbf{u}, \mathbf{v}] \mathbf{w}$ holds for all $\mathbf{u}, \mathbf{v}, \mathbf{w} \in \mathbb{V}_{S}$. First, we prove the left cancellation law which is given by the equation

$$
\ominus_{E} \mathbf{a} \oplus_{E}\left(\mathbf{a} \oplus_{E} \mathbf{b}\right)=\mathbf{b},
$$

for all $\mathbf{a}, \mathbf{b} \in \mathbb{V}_{s}$. Put $D_{\mathbf{u v}}=1+\frac{\mathbf{u} \cdot \mathbf{v}}{s^{2}}$ for any $\mathbf{u}, \mathbf{v} \in \mathbb{V}_{s}$ and put

$$
\begin{aligned}
\mathbf{x} & =\mathbf{a} \oplus_{E} \mathbf{b} \\
& =\frac{1}{D_{\mathbf{a b}}}\left\{\mathbf{a}+\frac{1}{\gamma_{\mathbf{a}}} \mathbf{b}+\frac{1}{s^{2}} \frac{\gamma_{\mathbf{a}}}{1+\gamma_{\mathbf{a}}}(\mathbf{a} \cdot \mathbf{b}) \mathbf{a}\right\} .
\end{aligned}
$$

Put $1+\frac{1}{s^{2}}((-\mathbf{a}) \cdot \mathbf{x})=D_{(-\mathbf{a}) \mathbf{x}}$. We have

$$
\begin{aligned}
\ominus_{E} \mathbf{a} \oplus_{E} \mathbf{x} & =\frac{1}{D_{(-\mathbf{a}) \mathbf{x}}}\left\{(-\mathbf{a})+\frac{1}{\gamma_{\mathbf{a}}} \mathbf{x}+\frac{1}{s^{2}} \frac{\gamma_{\mathbf{a}}}{1+\gamma_{\mathbf{a}}}(\mathbf{a} \cdot \mathbf{x}) \mathbf{a}\right\} \\
& =\frac{1}{D_{(-\mathbf{a}) \mathbf{x}}}\left\{(-\mathbf{a})+\frac{1}{\gamma_{\mathbf{a}} D_{\mathbf{a b}}}\left(\frac{1+\gamma_{\mathbf{a}} D_{\mathbf{a b}}}{1+\gamma_{\mathbf{a}}} \mathbf{a}+\frac{1}{\gamma_{\mathbf{a}}} \mathbf{b}\right)+\frac{1}{s^{2}} \frac{\gamma_{\mathbf{a}}}{1+\gamma_{\mathbf{a}}}(\mathbf{a} \cdot \mathbf{x}) \mathbf{a}\right\} .
\end{aligned}
$$

We compute,

$$
\begin{aligned}
D_{(-\mathbf{a}) \mathbf{x}} & =1+\frac{1}{s^{2}}((-\mathbf{a}) \cdot \mathbf{x}) \\
& =1-\frac{1}{D_{\mathbf{a b} s^{2}}}\left\{\|\mathbf{a}\|^{2}+\frac{1}{\gamma_{\mathbf{a}}}(\mathbf{a} \cdot \mathbf{b})+\frac{1}{s^{2}} \frac{\gamma_{\mathbf{a}}}{1+\gamma_{\mathbf{a}}}(\mathbf{a} \cdot \mathbf{b})\|\mathbf{a}\|^{2}\right\} \\
& =1-\frac{1}{D_{\mathbf{a b}}}\left\{\frac{\gamma_{\mathbf{a}}{ }^{2}-1}{\gamma_{\mathbf{a}}{ }^{2}}+\frac{1}{\gamma_{\mathbf{a}}} \frac{(\mathbf{a} \cdot \mathbf{b})}{s^{2}}+\frac{\gamma_{\mathbf{a}}}{1+\gamma_{\mathbf{a}}} \frac{(\mathbf{a} \cdot \mathbf{b})}{s^{2}} \frac{\gamma_{\mathbf{a}}{ }^{2}-1}{\gamma_{\mathbf{a}}{ }^{2}}\right\} \\
& =1-\frac{1}{D_{\mathbf{a b}}}\left\{1-\frac{1}{\gamma_{\mathbf{a}}{ }^{2}}+\frac{1}{\gamma_{\mathbf{a}}}\left(D_{\mathbf{a b}}-1\right)+\frac{\gamma_{\mathbf{a}}-1}{\gamma_{\mathbf{a}}}\left(D_{\mathbf{a b}}-1\right)\right\} \\
& =\frac{1}{\gamma_{\mathbf{a}}{ }^{2} D_{\mathbf{a b}}},
\end{aligned}
$$

and

$$
\frac{\mathbf{a} \cdot \mathbf{x}}{s^{2}}=1-D_{(-\mathbf{a}) \mathbf{x}}=\frac{\gamma_{\mathbf{a}}^{2} D_{\mathbf{a b}}-1}{\gamma_{\mathbf{a}}^{2} D_{\mathbf{a b}}}
$$

Hence we have

$$
\begin{aligned}
\ominus_{E} \mathbf{a} \oplus_{E}\left(\mathbf{a} \oplus_{E} \mathbf{b}\right) & =\ominus_{E} \mathbf{a} \oplus_{E} \mathbf{x} \\
& =\gamma_{\mathbf{a}}{ }^{2} D_{\mathbf{a} \mathbf{b}}\left\{(-\mathbf{a})+\frac{1}{\gamma_{\mathbf{a}} D_{\mathbf{a b}}}\left(\frac{1+\gamma_{\mathbf{a}} D_{\mathbf{a b}}}{1+\gamma_{\mathbf{a}}} \mathbf{a}+\frac{1}{\gamma_{\mathbf{a}}} \mathbf{b}\right)\right. \\
& \left.+\frac{\gamma_{\mathbf{a}}}{1+\gamma_{\mathbf{a}}} \frac{\gamma_{\mathbf{a}}{ }^{2} D_{\mathbf{a b}}-1}{\gamma_{\mathbf{a}}{ }^{2} D_{\mathbf{a} \mathbf{b}}} \mathbf{a}\right\} \\
& =-\gamma_{\mathbf{a}}{ }^{2} D_{\mathbf{a} \mathbf{b}} \mathbf{a}+\gamma_{\mathbf{a}}\left(\frac{1+\gamma_{\mathbf{a}} D_{\mathbf{a b}}}{1+\gamma_{\mathbf{a}}} \mathbf{a}+\frac{1}{\gamma_{\mathbf{a}}} \mathbf{b}\right)+\frac{\gamma_{\mathbf{a}}}{1+\gamma_{\mathbf{a}}}\left(\gamma_{\mathbf{a}}{ }^{2} D_{\mathbf{a b}}-1\right) \mathbf{a} \\
& =\mathbf{b}+\left(-\gamma_{\mathbf{a}}{ }^{2} D_{\mathbf{a b}}+\frac{\gamma_{\mathbf{a}}+\gamma_{\mathbf{a}}{ }^{2} D_{\mathbf{a b}}}{1+\gamma_{\mathbf{a}}}+\frac{\gamma_{\mathbf{a}}{ }^{3} D_{\mathbf{a b}}-\gamma_{\mathbf{a}}}{1+\gamma_{\mathbf{a}}}\right) \mathbf{a} \\
& =\mathbf{b} .
\end{aligned}
$$

Next, we prove the following equation

$$
\operatorname{gyr}[\mathbf{u}, \mathbf{v}] \mathbf{w}=\ominus_{E}\left(\mathbf{u} \oplus_{E} \mathbf{v}\right) \oplus_{E}\left(\mathbf{u} \oplus_{E}\left(\mathbf{v} \oplus_{E} \mathbf{w}\right)\right) .
$$


It is known in [5] ((2.84), (2.85)) that the Equation (14) can be rewritten as

$$
\operatorname{gyr}[\mathbf{u}, \mathbf{v}] \mathbf{w}=\mathbf{w}+\frac{\mathrm{A} \mathbf{u}+\mathrm{B} \mathbf{v}}{\mathrm{D}}
$$

by applying computer algebra, where

$$
\begin{aligned}
A & =-\frac{1}{s^{2}} \frac{\gamma_{\mathbf{u}}^{2}}{\gamma_{\mathbf{u}}+1}\left(\gamma_{\mathbf{v}}-1\right)(\mathbf{u} \cdot \mathbf{w})+\frac{1}{s^{2}} \gamma_{\mathbf{u}} \gamma_{\mathbf{v}}(\mathbf{v} \cdot \mathbf{w})+\frac{2}{s^{4}} \frac{\gamma_{\mathbf{u}}^{2} \gamma_{\mathbf{v}}^{2}}{\left(\gamma_{\mathbf{u}}+1\right)\left(\gamma_{\mathbf{v}}+1\right)}(\mathbf{u} \cdot \mathbf{v})(\mathbf{v} \cdot \mathbf{w}) \\
B & =-\frac{1}{s^{2}} \frac{\gamma_{\mathbf{v}}}{\gamma_{\mathbf{v}}+1}\left\{\gamma_{\mathbf{u}}\left(\gamma_{\mathbf{v}}+1\right)(\mathbf{u} \cdot \mathbf{w})+\left(\gamma_{\mathbf{u}}-1\right) \gamma_{\mathbf{v}}(\mathbf{v} \cdot \mathbf{w})\right\} \\
D & =\gamma_{\mathbf{u}} \gamma_{\mathbf{v}}\left(1+\frac{\mathbf{u} \cdot \mathbf{v}}{s^{2}}\right)+1
\end{aligned}
$$

We prove (15) without applying computer algebra. Put

$$
\begin{aligned}
\mathbf{z} & =\ominus_{E}\left(\mathbf{u} \oplus_{E} \mathbf{v}\right) \\
& =-\frac{1}{1+\frac{\mathbf{u} \cdot \mathbf{v}}{s^{2}}}\left\{\mathbf{u}+\frac{1}{\gamma_{\mathbf{u}}} \mathbf{v}+\frac{1}{s^{2}} \frac{\gamma_{\mathbf{u}}}{1+\gamma_{\mathbf{u}}}(\mathbf{u} \cdot \mathbf{v}) \mathbf{u}\right\} \\
& =-\frac{1+\gamma_{\mathbf{u}} D_{\mathbf{u v}}}{D_{\mathbf{u v}}\left(1+\gamma_{\mathbf{u}}\right)} \mathbf{u}-\frac{1}{\gamma_{\mathbf{u}} D_{\mathbf{u v}}} \mathbf{v} \\
\mathbf{x} & =\left(\mathbf{v} \oplus_{E} \mathbf{w}\right) \\
& =\frac{1+\gamma_{\mathbf{v}} D_{\mathbf{v w}}}{D_{\mathbf{v w}}\left(1+\gamma_{\mathbf{v}}\right)} \mathbf{v}+\frac{1}{\gamma_{\mathbf{v}} D_{\mathbf{v} \mathbf{w}}} \mathbf{w} .
\end{aligned}
$$

Put also

$$
\begin{aligned}
\mathbf{y} & =\mathbf{u} \oplus_{E}\left(\mathbf{v} \oplus_{E} \mathbf{w}\right) \\
& =\mathbf{u} \oplus_{E} \mathbf{x} \\
& =\frac{1+\gamma_{\mathbf{u}} D_{\mathbf{u x}}}{D_{\mathbf{u x}}\left(1+\gamma_{\mathbf{u}}\right)} \mathbf{u}+\frac{1}{\gamma_{\mathbf{u}} D_{\mathbf{u x}}} \mathbf{x} \\
& =\frac{1+\gamma_{\mathbf{u}} D_{\mathbf{u x}}}{D_{\mathbf{u x}}\left(1+\gamma_{\mathbf{u}}\right)} \mathbf{u}+\frac{1+\gamma_{\mathbf{v}} D_{\mathbf{v} \mathbf{w}}}{D_{\mathbf{u x}} D_{\mathbf{v} \mathbf{w}} \gamma_{\mathbf{u}}\left(1+\gamma_{\mathbf{v}}\right)} \mathbf{v}+\frac{1}{D_{\mathbf{u x}} D_{\mathbf{v} \mathbf{w}} \gamma_{\mathbf{u}} \gamma_{\mathbf{v}}} \mathbf{w} .
\end{aligned}
$$

Then $\operatorname{gyr}[\mathbf{u}, \mathbf{v}] \mathbf{w}$ is given by the following:

$$
\begin{aligned}
\operatorname{gyr}[\mathbf{u}, \mathbf{v}] \mathbf{w} & =\mathbf{z} \oplus_{E} \mathbf{y} \\
& =\frac{1+\gamma_{\mathbf{z}} D_{\mathbf{z y}}}{D_{\mathbf{z y}}\left(1+\gamma_{\mathbf{z}}\right)} \mathbf{z}+\frac{1}{\gamma_{\mathbf{z}} D_{\mathbf{z y}}} \mathbf{y} \\
& =-\frac{\left(1+\gamma_{\mathbf{z}} D_{\mathbf{z y}}\right)\left(1+\gamma_{\mathbf{u}} D_{\mathbf{u v}}\right)}{D_{\mathbf{z y}} D_{\mathbf{u v}}\left(1+\gamma_{\mathbf{z}}\right)\left(1+\gamma_{\mathbf{u}}\right)} \mathbf{u}-\frac{1+\gamma_{\mathbf{z}} D_{\mathbf{z y}}}{D_{\mathbf{z y}} D_{\mathbf{u v}}\left(1+\gamma_{\mathbf{z}}\right) \gamma_{\mathbf{u}}} \mathbf{v} \\
& +\frac{1+\gamma_{\mathbf{u}} D_{\mathbf{u x}}}{D_{\mathbf{z y}} D_{\mathbf{u x}} \gamma_{\mathbf{z}}\left(1+\gamma_{\mathbf{u}}\right)} \mathbf{u}+\frac{1+\gamma_{\mathbf{v}} D_{\mathbf{v} \mathbf{w}}}{D_{\mathbf{z y}} D_{\mathbf{u x}} \gamma_{\mathbf{z}} \gamma_{\mathbf{u}}\left(1+\gamma_{\mathbf{v}}\right)} \mathbf{v} \\
& +\frac{1}{D_{\mathbf{z y}} D_{\mathbf{u x}} D_{\mathbf{v w}} \gamma_{\mathbf{z}} \gamma_{\mathbf{u}} \gamma_{\mathbf{v}}} \mathbf{w} .
\end{aligned}
$$

We will calculate each coefficient of $\mathbf{u}, \mathbf{v}, \mathbf{w}$ of the equation above. 
We prove that the coefficient of $\mathbf{w}$ is 1, i.e., $D_{\mathbf{z y}} D_{\mathbf{u x}} D_{\mathbf{v w}} \gamma_{\mathbf{u}} \gamma_{\mathbf{v}} \gamma_{\mathbf{z}}$ is 1 . The equation $\frac{\mathbf{a} \cdot \mathbf{b}}{s^{2}}=D_{\mathbf{a b}}-1$ holds for all $\mathbf{a}, \mathbf{b} \in \mathbb{V}_{s}$. Applying this equation, we have

$$
\begin{aligned}
& D_{\mathbf{u x}}=1+\frac{\mathbf{u} \cdot \mathbf{x}}{s^{2}} \\
& =1+\frac{1}{s^{2}}\left\{\frac{1+\gamma_{\mathbf{v}} D_{\mathbf{v w}}}{D_{\mathbf{v w}}\left(1+\gamma_{\mathbf{v}}\right)}(\mathbf{u} \cdot \mathbf{v})+\frac{1}{\gamma_{\mathbf{v}} D_{\mathbf{v w}}}(\mathbf{u} \cdot \mathbf{w})\right\} \\
& =\frac{D_{\mathbf{v w}} \gamma_{\mathbf{v}}\left(1+\gamma_{\mathbf{v}}\right)+\gamma_{\mathbf{v}}\left(1+\gamma_{\mathbf{v}} D_{\mathbf{v w}}\right)\left(D_{\mathbf{u v}}-1\right)+\left(1+\gamma_{\mathbf{v}}\right)\left(D_{\mathbf{u w}}-1\right)}{D_{\mathbf{v w}} \gamma_{\mathbf{v}}\left(1+\gamma_{\mathbf{v}}\right)} \\
& =\frac{\gamma_{\mathbf{v}}^{2} D_{\mathbf{u v}} D_{\mathbf{v w}}+\gamma_{\mathbf{v}} D_{\mathbf{u v}}+\gamma_{\mathbf{v}} D_{\mathbf{v w}}+\gamma_{\mathbf{v}} D_{\mathbf{u w}}+D_{\mathbf{u w}}-2 \gamma_{\mathbf{v}}-1}{D_{\mathbf{v w}} \gamma_{\mathbf{v}}\left(1+\gamma_{\mathbf{v}}\right)}, \\
& D_{\mathbf{z y}}=1+\frac{\mathbf{z} \cdot \mathbf{y}}{s^{2}} \\
& =1-\frac{1}{s^{2}}\left\{\frac{\left(1+\gamma_{\mathbf{u}} D_{\mathbf{u v}}\right)\left(1+\gamma_{\mathbf{u}} D_{\mathbf{u x}}\right)}{D_{\mathbf{u v}} D_{\mathbf{u x}}\left(1+\gamma_{\mathbf{u}}\right)^{2}}\|\mathbf{u}\|^{2}+\frac{\left(1+\gamma_{\mathbf{u}} D_{\mathbf{u v}}\right)\left(1+\gamma_{\mathbf{v}} D_{\mathbf{v w}}\right)}{D_{\mathbf{u v}} D_{\mathbf{u x}} D_{\mathbf{v w}} \gamma_{\mathbf{u}}\left(1+\gamma_{\mathbf{u}}\right)\left(1+\gamma_{\mathbf{v}}\right)}(\mathbf{u} \cdot \mathbf{v})\right. \\
& +\frac{1+\gamma_{\mathbf{u}} D_{\mathbf{u v}}}{D_{\mathbf{u v}} D_{\mathbf{u x}} D_{\mathbf{v w}} \gamma_{\mathbf{u}} \gamma_{\mathbf{v}}\left(1+\gamma_{\mathbf{u}}\right)}(\mathbf{u} \cdot \mathbf{w})+\frac{1+\gamma_{\mathbf{u}} D_{\mathbf{u x}}}{D_{\mathbf{u v}} D_{\mathbf{u x}} \gamma_{\mathbf{u}}\left(1+\gamma_{\mathbf{u}}\right)}(\mathbf{u} \cdot \mathbf{v}) \\
& \left.+\frac{1+\gamma_{\mathbf{v}} D_{\mathbf{v w}}}{D_{\mathbf{u v}} D_{\mathbf{u x}} D_{\mathbf{v w}} \gamma_{\mathbf{u}}^{2}\left(1+\gamma_{\mathbf{v}}\right)}\|\mathbf{v}\|^{2}+\frac{\mathbf{v} \cdot \mathbf{w}}{D_{\mathbf{u v}} D_{\mathbf{u x}} D_{\mathbf{v w}} \gamma_{\mathbf{u}}^{2} \gamma_{\mathbf{v}}}\right\} \text {. }
\end{aligned}
$$

Multiplying $D_{\mathbf{u x}} D_{\mathbf{v w}}$ from the right-hand side of the last equation, and applying the gamma factor, we have

$$
\begin{aligned}
& D_{\mathbf{z y}}\left(D_{\mathbf{u x}} D_{\mathbf{v w}}\right) \\
& =D_{\mathbf{u x}} D_{\mathbf{v w}}-\frac{\left(1+\gamma_{\mathbf{u}} D_{\mathbf{u v}}\right)\left(1+\gamma_{\mathbf{u}} D_{\mathbf{u x}}\right)\left(\gamma_{\mathbf{u}}-1\right) D_{\mathbf{v w}}}{D_{\mathbf{u v}}\left(1+\gamma_{\mathbf{u}}\right) \gamma_{\mathbf{u}}{ }^{2}} \\
& -\frac{\left(1+\gamma_{\mathbf{u}} D_{\mathbf{u v}}\right)\left(1+\gamma_{\mathbf{v}} D_{\mathbf{v w}}\right)\left(D_{\mathbf{u v}}-1\right)}{D_{\mathbf{u v}} \gamma_{\mathbf{u}}\left(1+\gamma_{\mathbf{u}}\right)\left(1+\gamma_{\mathbf{v}}\right)}-\frac{\left(1+\gamma_{\mathbf{u}} D_{\mathbf{u v}}\right)\left(D_{\mathbf{u w}}-1\right)}{D_{\mathbf{u v}} \gamma_{\mathbf{u}} \gamma_{\mathbf{v}}\left(1+\gamma_{\mathbf{u}}\right)} \\
& -\frac{\left(1+\gamma_{\mathbf{u}} D_{\mathbf{u x}}\right)\left(D_{\mathbf{u v}}-1\right) D_{\mathbf{v w}}}{D_{\mathbf{u v}} \gamma_{\mathbf{u}}\left(1+\gamma_{\mathbf{u}}\right)}-\frac{\left(1+\gamma_{\mathbf{v}} D_{\mathbf{v w}}\right)\left(\gamma_{\mathbf{v}}-1\right)}{D_{\mathbf{u v}} \gamma_{\mathbf{u}} \gamma_{\mathbf{v}}{ }^{2}}-\frac{D_{\mathbf{v w}}-1}{D_{\mathbf{u v}} \gamma_{\mathbf{u}}{ }^{2} \gamma_{\mathbf{v}}}
\end{aligned}
$$

Dividing the common denominator $D_{\mathbf{u v}} \gamma_{\mathbf{u}}{ }^{2} \gamma_{\mathbf{v}}{ }^{2}\left(1+\gamma_{\mathbf{u}}\right)\left(1+\gamma_{\mathbf{v}}\right)$ and multiplying $\gamma_{\mathbf{u}} \gamma_{\mathbf{v}} \gamma_{\mathbf{z}}$ to $D_{\mathbf{z y}} D_{\mathbf{u x}} D_{\mathbf{v w}}$, where $\gamma_{\mathbf{z}}=\gamma_{\ominus_{\mathbf{E}}\left(\mathbf{u} \oplus_{\mathbf{E}} \mathbf{v}\right)}=\gamma_{\mathbf{u} \oplus_{\mathbf{E}} \mathbf{v}}=D_{\mathbf{u v}} \gamma_{\mathbf{u}} \gamma_{\mathbf{v}}$, we have

$$
\begin{aligned}
& D_{\mathbf{z y}} D_{\mathbf{u x}} D_{\mathbf{v w}} \gamma_{\mathbf{u}} \gamma_{\mathbf{v}} \gamma_{\mathbf{z}} \\
& =\frac{1}{\left(1+\gamma_{\mathbf{u}}\right)\left(1+\gamma_{\mathbf{v}}\right)}\left\{D_{\mathbf{u v}} D_{\mathbf{u x}} D_{\mathbf{v} \mathbf{w}} \gamma_{\mathbf{u}}{ }^{2} \gamma_{\mathbf{v}}{ }^{2}\left(1+\gamma_{\mathbf{u}}\right)\left(1+\gamma_{\mathbf{v}}\right)\right. \\
& -\left(1+\gamma_{\mathbf{u}} D_{\mathbf{u v}}\right)\left(1+\gamma_{\mathbf{u}} D_{\mathbf{u x}}\right)\left(\gamma_{\mathbf{u}}-1\right) D_{\mathbf{v w}} \gamma_{\mathbf{v}}{ }^{2}\left(1+\gamma_{\mathbf{v}}\right) \\
& -\left(1+\gamma_{\mathbf{u}} D_{\mathbf{u v}}\right)\left(1+\gamma_{\mathbf{v}} D_{\mathbf{v w}}\right)\left(D_{\mathbf{u v}}-1\right) \gamma_{\mathbf{u}} \gamma_{\mathbf{v}}{ }^{2}-\left(1+\gamma_{\mathbf{u}} D_{\mathbf{u v}}\right)\left(D_{\mathbf{u w}}-1\right)\left(1+\gamma_{\mathbf{v}}\right) \gamma_{\mathbf{u}} \gamma_{\mathbf{v}} \\
& -\left(1+\gamma_{\mathbf{u}} D_{\mathbf{u} \mathbf{x}}\right)\left(D_{\mathbf{u v}}-1\right) D_{\mathbf{v w}} \gamma_{\mathbf{u}} \gamma_{\mathbf{v}}{ }^{2}-\left(1+\gamma_{\mathbf{v}} D_{\mathbf{v w}}\right)\left(\gamma_{\mathbf{v}}-1\right)\left(1+\gamma_{\mathbf{u}}\right)\left(1+\gamma_{\mathbf{v}}\right) \\
& \left.-\left(D_{\mathbf{v} \mathbf{w}}-1\right) \gamma_{\mathbf{v}}\left(1+\gamma_{\mathbf{u}}\right)\left(1+\gamma_{\mathbf{v}}\right)\right\} .
\end{aligned}
$$


We compute $\{\cdot\}$ of the Equation (16).

$$
\begin{aligned}
& \{\cdot\}=D_{\mathbf{u v}} D_{\mathbf{u x}} D_{\mathbf{v w}} \gamma_{\mathbf{u}}{ }^{2} \gamma_{\mathbf{v}}{ }^{2}+D_{\mathbf{u v}} D_{\mathbf{u x}} D_{\mathbf{v w}} \gamma_{\mathbf{u}}{ }^{3} \gamma_{\mathbf{v}}{ }^{2}+D_{\mathbf{u v}} D_{\mathbf{u x}} D_{\mathbf{v w}} \gamma_{\mathbf{u}}{ }^{2} \gamma_{\mathbf{v}}{ }^{3} \\
& +D_{\mathbf{u v}} D_{\mathbf{u x}} D_{\mathbf{v w}} \gamma_{\mathbf{u}}{ }^{3} \gamma_{\mathbf{v}}{ }^{3}-D_{\mathbf{v w}} \gamma_{\mathbf{u}} \gamma_{\mathbf{v}}{ }^{2}+D_{\mathbf{v w}} \gamma_{\mathbf{v}}{ }^{3}-D_{\mathbf{v w}} \gamma_{\mathbf{u}} \gamma_{\mathbf{v}}{ }^{3}+D_{\mathbf{v w}} \gamma_{\mathbf{v}}{ }^{2} \\
& -D_{\mathbf{v w}} D_{\mathbf{u x}} \gamma_{\mathbf{u}}{ }^{2} \gamma_{\mathbf{v}}{ }^{2}+D_{\mathbf{v w}} D_{\mathbf{u x}} \gamma_{\mathbf{u}} \gamma_{\mathbf{v}}{ }^{3}-D_{\mathbf{v w}} D_{\mathbf{u x}} \gamma_{\mathbf{u}}{ }^{2} \gamma_{\mathbf{v}}{ }^{3}+D_{\mathbf{v w}} D_{\mathbf{u x}} \gamma_{\mathbf{u}} \gamma_{\mathbf{v}}{ }^{2} \\
& -D_{\mathbf{u v}} D_{\mathbf{v w}} \gamma_{\mathbf{u}}{ }^{2} \gamma_{\mathbf{v}}{ }^{2}+D_{\mathbf{u v}} D_{\mathbf{v w}} \gamma_{\mathbf{u}} \gamma_{\mathbf{v}}{ }^{3}-D_{\mathbf{u v}} D_{\mathbf{v w}} \gamma_{\mathbf{u}}{ }^{2} \gamma_{\mathbf{v}}{ }^{3}+D_{\mathbf{u v}} D_{\mathbf{v w}} \gamma_{\mathbf{u}} \gamma_{\mathbf{v}}{ }^{2} \\
& -D_{\mathbf{u v}} D_{\mathbf{v w}} D_{\mathbf{u x}} \gamma_{\mathbf{u}}{ }^{3} \gamma_{\mathbf{v}}{ }^{2}+D_{\mathbf{u v}} D_{\mathbf{v w}} D_{\mathbf{u x}} \gamma_{\mathbf{u}}{ }^{2} \gamma_{\mathbf{v}}{ }^{3}-D_{\mathbf{u v}} D_{\mathbf{v w}} D_{\mathbf{u x}} \gamma_{\mathbf{u}}{ }^{3} \gamma_{\mathbf{v}}{ }^{3}
\end{aligned}
$$

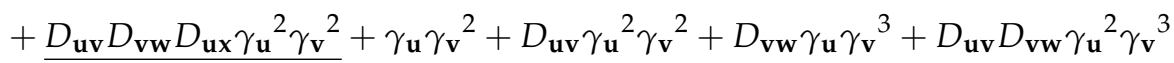

$$
\begin{aligned}
& -D_{\mathbf{u v}} \gamma_{\mathbf{u}} \gamma_{\mathbf{v}}{ }^{2}-D_{\mathbf{u v}}^{2} \gamma_{\mathbf{u}}{ }^{2} \gamma_{\mathbf{v}}{ }^{2}-D_{\mathbf{u v}} D_{\mathbf{v w}} \gamma_{\mathbf{u}} \gamma_{\mathbf{v}}{ }^{3}-D_{\mathbf{u v}}^{2} D_{\mathbf{v w}} \gamma_{\mathbf{u}}{ }^{2} \gamma_{\mathbf{v}}{ }^{3} \\
& +\gamma_{\mathbf{u}} \gamma_{\mathbf{v}}+\gamma_{\mathbf{u}} \gamma_{\mathbf{v}}{ }^{2}+D_{\mathbf{u v}} \gamma_{\mathbf{u}}^{2} \gamma_{\mathbf{v}}+D_{\mathbf{u v}} \gamma_{\mathbf{u}}^{2} \gamma_{\mathbf{v}}^{2} \\
& -D_{\mathbf{u w}} \gamma_{\mathbf{u}} \gamma_{\mathbf{v}}-D_{\mathbf{u w}} \gamma_{\mathbf{u}} \gamma_{\mathbf{v}}{ }^{2}-D_{\mathbf{u w}} D_{\mathbf{u v}} \gamma_{\mathbf{u}}{ }^{2} \gamma_{\mathbf{v}}-D_{\mathbf{u w}} D_{\mathbf{u v}} \gamma_{\mathbf{u}}{ }^{2} \gamma_{\mathbf{v}}{ }^{2} \\
& +D_{\mathbf{v w}} \gamma_{\mathbf{u}} \gamma_{\mathbf{v}}^{2}+D_{\mathbf{v w}} \gamma_{\mathbf{u}} \gamma_{\mathbf{v}}{ }^{3}+D_{\mathbf{v w}} D_{\mathbf{u x}} \gamma_{\mathbf{u}}{ }^{2} \gamma_{\mathbf{v}}{ }^{2}+D_{\mathbf{v w}} D_{\mathbf{u x}} \gamma_{\mathbf{u}}{ }^{2} \gamma_{\mathbf{v}}{ }^{3} \\
& -D_{\mathbf{u v}} D_{\mathbf{v w}} \gamma_{\mathbf{u}} \gamma_{\mathbf{v}}{ }^{2}-D_{\mathbf{u v}} D_{\mathbf{v w}} \gamma_{\mathbf{u}} \gamma_{\mathbf{v}}{ }^{3}-D_{\mathbf{u v}} D_{\mathbf{v w}} D_{\mathbf{u x}} \gamma_{\mathbf{u}}{ }^{2} \gamma_{\mathbf{v}}{ }^{2} \\
& -D_{\mathbf{u v}} D_{\mathbf{v w}} D_{\mathbf{u x}} \gamma_{\mathbf{u}}{ }^{2} \gamma_{\mathbf{v}}{ }^{3}-\gamma_{\mathbf{v}}{ }^{2}+1-\gamma_{\mathbf{u}} \gamma_{\mathbf{v}}{ }^{2}+\gamma_{\mathbf{u}}-D_{\mathbf{v w}} \gamma_{\mathbf{v}}{ }^{3}+D_{\mathbf{v w}} \gamma_{\mathbf{v}} \\
& -D_{\mathbf{v w}} \gamma_{\mathbf{u}} \gamma_{\mathbf{v}}{ }^{3}+D_{\mathbf{v w}} \gamma_{\mathbf{u}} \gamma_{\mathbf{v}}+\gamma_{\mathbf{v}}+\gamma_{\mathbf{u}} \gamma_{\mathbf{v}}+\gamma_{\mathbf{v}}{ }^{2}+\gamma_{\mathbf{u}} \gamma_{\mathbf{v}}{ }^{2}-D_{\mathbf{v w}} \gamma_{\mathbf{v}}-D_{\mathbf{v w}} \gamma_{\mathbf{u}} \gamma_{\mathbf{v}} \\
& -D_{\mathbf{v w}} \gamma_{\mathbf{v}}^{2}-D_{\mathbf{v w}} \gamma_{\mathbf{u}} \gamma_{\mathbf{v}}^{2} \text {. }
\end{aligned}
$$

Applying

$$
\begin{aligned}
D_{\mathbf{u x}} D_{\mathbf{v w}} \gamma_{\mathbf{v}}\left(1+\gamma_{\mathbf{v}}\right) & =\gamma_{\mathbf{v}}^{2} D_{\mathbf{u v}} D_{\mathbf{v w}}+\gamma_{\mathbf{v}} D_{\mathbf{u v}}+\gamma_{\mathbf{v}} D_{\mathbf{v w}} \\
& +\gamma_{\mathbf{v}} D_{\mathbf{u w}}+D_{\mathbf{u w}}-2 \gamma_{\mathbf{v}}-1
\end{aligned}
$$

for underline items, we infer that

$$
\begin{aligned}
& \underline{D_{\mathbf{u v}} D_{\mathbf{v w}} D_{\mathbf{u x}} \gamma_{\mathbf{u}}{ }^{2} \gamma_{\mathbf{v}}{ }^{3}}+\underline{D_{\mathbf{u v}} D_{\mathbf{v w}} D_{\mathbf{u x}} \gamma_{\mathbf{u}}{ }^{2} \gamma_{\mathbf{v}}{ }^{2}} \\
& =D_{\mathbf{u v}} \gamma_{\mathbf{u}}{ }^{2} \gamma_{\mathbf{v}} D_{\mathbf{u x}} D_{\mathbf{v w}} \gamma_{\mathbf{v}}\left(1+\gamma_{\mathbf{v}}\right) \\
& =D_{\mathbf{u v}}^{2} D_{\mathbf{v w}} \gamma_{\mathbf{u}}{ }^{2} \gamma_{\mathbf{v}}{ }^{3}+D_{\mathbf{u v}}^{2} \gamma_{\mathbf{u}}{ }^{2} \gamma_{\mathbf{v}}{ }^{2}+D_{\mathbf{u v}} D_{\mathbf{v w}} \gamma_{\mathbf{u}}{ }^{2} \gamma_{\mathbf{v}}{ }^{2}+D_{\mathbf{u w}} D_{\mathbf{u v}} \gamma_{\mathbf{u}}{ }^{2} \gamma_{\mathbf{v}}{ }^{2} \\
& +D_{\mathbf{u w}} D_{\mathbf{u v}} \gamma_{\mathbf{u}}^{2} \gamma_{\mathbf{v}}-2 D_{\mathbf{u v}} \gamma_{\mathbf{u}}^{2} \gamma_{\mathbf{v}}^{2}+D_{\mathbf{u v}} \gamma_{\mathbf{u}}^{2}
\end{aligned}
$$

So, we have

$$
\begin{aligned}
\{\cdot\} & =-D_{\mathbf{v w}} \gamma_{\mathbf{u}} \gamma_{\mathbf{v}}{ }^{2}-D_{\mathbf{v w}} \gamma_{\mathbf{u}} \gamma_{\mathbf{v}}{ }^{3}-D_{\mathbf{u x}} D_{\mathbf{v w}} \gamma_{\mathbf{v}}\left(1+\gamma_{\mathbf{v}}\right)\left(\gamma_{\mathbf{u}}{ }^{2} \gamma_{\mathbf{v}}-\gamma_{\mathbf{u}} \gamma_{\mathbf{v}}\right) \\
& +2 \gamma_{\mathbf{u}} \gamma_{\mathbf{v}}{ }^{2}+D_{\mathbf{v} \mathbf{w}} \gamma_{\mathbf{u}} \gamma_{\mathbf{v}}{ }^{3}-D_{\mathbf{u v}} \gamma_{\mathbf{u}} \gamma_{\mathbf{v}}{ }^{2}-D_{\mathbf{u v}} D_{\mathbf{v w}} \gamma_{\mathbf{u}} \gamma_{\mathbf{v}}{ }^{3} \\
& +\gamma_{\mathbf{u}} \gamma_{\mathbf{v}}-D_{\mathbf{u w}} \gamma_{\mathbf{u}} \gamma_{\mathbf{v}}-D_{\mathbf{u w}} \gamma_{\mathbf{u}} \gamma_{\mathbf{v}}{ }^{2}+D_{\mathbf{u} \mathbf{x}} D_{\mathbf{v} \mathbf{w}} \gamma_{\mathbf{v}}\left(1+\gamma_{\mathbf{v}}\right) \gamma_{\mathbf{u}}{ }^{2} \gamma_{\mathbf{v}} \\
& +1+\gamma_{\mathbf{u}}+\gamma_{\mathbf{v}}+\gamma_{\mathbf{u}} \gamma_{\mathbf{v}} \\
& =\left(1+\gamma_{\mathbf{u}}\right)\left(1+\gamma_{\mathbf{v}}\right)
\end{aligned}
$$

hence we have $D_{\mathbf{z y}} D_{\mathbf{u x}} D_{\mathbf{v w}} \gamma_{\mathbf{u}} \gamma_{\mathbf{v}} \gamma_{\mathbf{z}}=1$.

Next, we prove that coefficient of $\mathbf{u}$ is $\frac{A}{D}$. 
We have $1+\gamma_{\mathbf{z}}=1+D_{\mathbf{u v}} \gamma_{\mathbf{u}} \gamma_{\mathbf{v}}=D$. Then we compute the coefficient of $\mathbf{u}$ applying the equation $D_{\mathbf{z y}} D_{\mathbf{u x}} D_{\mathbf{v w}} \gamma_{\mathbf{u}} \gamma_{\mathbf{v}} \gamma_{\mathbf{z}}=1$.

$$
\begin{aligned}
& -\frac{\left(1+\gamma_{\mathbf{z}} D_{\mathbf{z y}}\right)\left(1+\gamma_{\mathbf{u}} D_{\mathbf{u v}}\right)}{D_{\mathbf{z y}} D_{\mathbf{u v}}\left(1+\gamma_{\mathbf{z}}\right)\left(1+\gamma_{\mathbf{u}}\right)}+\frac{1+\gamma_{\mathbf{u}} D_{\mathbf{u x}}}{D_{\mathbf{z y}} D_{\mathbf{u x}} \gamma_{\mathbf{z}}\left(1+\gamma_{\mathbf{u}}\right)} \\
& =\frac{-\left(1+\gamma_{\mathbf{z}} D_{\mathbf{z y}}\right)\left(1+\gamma_{\mathbf{u}} D_{\mathbf{u v}}\right) \gamma_{\mathbf{z}} D_{\mathbf{u x}}+\left(1+\gamma_{\mathbf{u}} D_{\mathbf{u x}}\right)\left(1+\gamma_{\mathbf{z}}\right) D_{\mathbf{u v}}}{D_{\mathbf{z y}} D_{\mathbf{u x}} D_{\mathbf{u v}} D \gamma_{\mathbf{z}}\left(1+\gamma_{\mathbf{u}}\right)} \\
& =\frac{D_{\mathbf{v w}} \gamma_{\mathbf{u}} \gamma_{\mathbf{v}}}{D D_{\mathbf{u v}}\left(1+\gamma_{\mathbf{u}}\right)}\left\{-\left(D_{\mathbf{u x}} \gamma_{\mathbf{z}}+D_{\mathbf{u x}} D_{\mathbf{u v}} \gamma_{\mathbf{u}} \gamma_{\mathbf{z}}+D_{\mathbf{z y}} D_{\mathbf{u x}} \gamma_{\mathbf{z}}^{2}+D_{\mathbf{z y}} D_{\mathbf{u x}} D_{\mathbf{u v}} \gamma_{\mathbf{z}}^{2} \gamma_{\mathbf{u}}\right)\right. \\
& \left.+D_{\mathbf{u v}}+D_{\mathbf{u v}} \gamma_{\mathbf{z}}+D_{\mathbf{u x}} D_{\mathbf{u v}} \gamma_{\mathbf{u}}+D_{\mathbf{u x}} D_{\mathbf{u v}} \gamma_{\mathbf{u}} \gamma_{\mathbf{z}}\right\} \\
& =\frac{D_{\mathbf{v w}} \gamma_{\mathbf{u}} \gamma_{\mathbf{v}}}{D D_{\mathbf{u v}}\left(1+\gamma_{\mathbf{u}}\right)}\left\{D_{\mathbf{u v}}+D_{\mathbf{u v}}^{2} \gamma_{\mathbf{u}} \gamma_{\mathbf{v}}+D_{\mathbf{u x}} D_{\mathbf{u v}} \gamma_{\mathbf{u}}\left(1-\gamma_{\mathbf{v}}\right)-\frac{D_{\mathbf{u v}}}{D_{\mathbf{v w}}}-\frac{D_{\mathbf{u v}}^{2} \gamma_{\mathbf{u}}}{D_{\mathbf{v w}}}\right\} \\
& =\frac{\gamma_{\mathbf{u}} \gamma_{\mathbf{v}}}{D\left(1+\gamma_{\mathbf{u}}\right)}\left\{D_{\mathbf{v w}}+D_{\mathbf{u v}} D_{\mathbf{v w}} \gamma_{\mathbf{u}} \gamma_{\mathbf{v}}-1-\gamma_{\mathbf{u}} D_{\mathbf{u v}}\right. \\
& \left.+\gamma_{\mathbf{u}}\left(1-\gamma_{\mathbf{v}}\right) \frac{\gamma_{\mathbf{v}}^{2} D_{\mathbf{u v}} D_{\mathbf{v w}}+\gamma_{\mathbf{v}} D_{\mathbf{u v}}+\gamma_{\mathbf{v}} D_{\mathbf{v w}}+\gamma_{\mathbf{v}} D_{\mathbf{u w}}+D_{\mathbf{u w}}-2 \gamma_{\mathbf{v}}-1}{\gamma_{\mathbf{v}}\left(1+\gamma_{\mathbf{v}}\right)}\right\} \\
& =\frac{\gamma_{\mathbf{u}} \gamma_{\mathbf{v}}}{D\left(1+\gamma_{\mathbf{u}}\right)}\left\{-\frac{\gamma_{\mathbf{u}}}{\gamma_{\mathbf{v}}}\left(\gamma_{\mathbf{v}}-1\right) D_{\mathbf{u w}}+\left(1-\frac{\gamma_{\mathbf{u}}\left(\gamma_{\mathbf{v}}-1\right)}{1+\gamma_{\mathbf{v}}}\right) D_{\mathbf{v w}}\right. \\
& \left.-\left(1+\frac{\gamma_{\mathbf{v}}-1}{1+\gamma_{\mathbf{v}}}\right) \gamma_{\mathbf{u}} D_{\mathbf{u v}}+\left(1-\frac{\gamma_{\mathbf{v}}-1}{1+\gamma_{\mathbf{v}}}\right) \gamma_{\mathbf{u}} \gamma_{\mathbf{v}} D_{\mathbf{u v}} D_{\mathbf{v w}}-1+\frac{\gamma_{\mathbf{u}}\left(\gamma_{\mathbf{v}}-1\right)\left(2 \gamma_{\mathbf{v}}+1\right)}{\gamma_{\mathbf{v}}\left(1+\gamma_{\mathbf{v}}\right)}\right\}
\end{aligned}
$$

By $D_{\mathbf{u v}}=1+\frac{\mathbf{u} \cdot \mathbf{v}}{s^{2}}$,

$$
\begin{aligned}
& =\frac{1}{D}\left\{-\frac{1}{s^{2}} \frac{\gamma_{\mathbf{u}}{ }^{2}}{\gamma_{\mathbf{v}}+1}\left(\gamma_{\mathbf{u}}-1\right)(\mathbf{u} \cdot \mathbf{v})-\frac{\gamma_{\mathbf{u}}{ }^{2}}{\gamma_{\mathbf{v}}+1}\left(\gamma_{\mathbf{v}}-1\right)\right. \\
& +\frac{1}{s^{2}} \frac{\gamma_{\mathbf{u}} \gamma_{\mathbf{v}}\left(1+\gamma_{\mathbf{u}}+\gamma_{\mathbf{v}}-\gamma_{\mathbf{u}} \gamma_{\mathbf{v}}\right)}{\left(\gamma_{\mathbf{u}}+1\right)\left(\gamma_{\mathbf{v}}+1\right)}(\mathbf{v} \cdot \mathbf{w})+\frac{\gamma_{\mathbf{u}} \gamma_{\mathbf{v}}\left(1+\gamma_{\mathbf{u}}+\gamma_{\mathbf{v}}-\gamma_{\mathbf{u}} \gamma_{\mathbf{v}}\right)}{\left(\gamma_{\mathbf{u}}+1\right)\left(\gamma_{\mathbf{v}}+1\right)} \\
& -\frac{1}{s^{2}} \frac{2 \gamma_{\mathbf{u}}{ }^{2} \gamma_{\mathbf{v}}{ }^{2}}{\left(\gamma_{\mathbf{u}}+1\right)\left(\gamma_{\mathbf{v}}+1\right)}(\mathbf{u} \cdot \mathbf{v})-\frac{1}{s^{2}} \frac{2 \gamma_{\mathbf{u}}{ }^{2} \gamma_{\mathbf{v}}{ }^{2}}{\left(\gamma_{\mathbf{u}}+1\right)\left(\gamma_{\mathbf{v}}+1\right)} \\
& +\frac{2 \gamma_{\mathbf{u}}{ }^{2} \gamma_{\mathbf{v}}{ }^{2}}{\left(\gamma_{\mathbf{u}}+1\right)\left(\gamma_{\mathbf{v}}+1\right)}\left(1+\frac{(\mathbf{u} \cdot \mathbf{v})}{s^{2}}+\frac{(\mathbf{v} \cdot \mathbf{w})}{s^{2}}+\frac{1}{s^{4}}(\mathbf{u} \cdot \mathbf{v})(\mathbf{v} \cdot \mathbf{w})\right) \\
& \left.-\frac{\gamma_{\mathbf{u}} \gamma_{\mathbf{v}}}{1+\gamma_{\mathbf{u}}}+\frac{\gamma_{\mathbf{u}}{ }^{2}\left(2 \gamma_{\mathbf{v}}{ }^{2}-\gamma_{\mathbf{v}}-1\right)}{\left(\gamma_{\mathbf{u}}+1\right)\left(\gamma_{\mathbf{v}}+1\right)}\right\} \\
& =\frac{1}{D}\left\{-\frac{1}{s^{2}} \frac{\gamma_{\mathbf{u}}{ }^{2}}{\gamma_{\mathbf{v}}+1}\left(\gamma_{\mathbf{v}}-1\right)(\mathbf{u} \cdot \mathbf{v})+\frac{1}{s^{2}} \gamma_{\mathbf{u}} \gamma_{\mathbf{v}}(\mathbf{v} \cdot \mathbf{w})+\frac{2}{s^{4}} \frac{\gamma_{\mathbf{u}}{ }^{2} \gamma_{\mathbf{v}}{ }^{2}}{\left(\gamma_{\mathbf{u}}+1\right)\left(\gamma_{\mathbf{v}}+1\right)}(\mathbf{u} \cdot \mathbf{v})(\mathbf{v} \cdot \mathbf{w})\right\} \\
& =\frac{A}{D} .
\end{aligned}
$$

Finally, we prove that the coefficient of $\mathbf{v}$ is $\frac{B}{D}$.

$$
\begin{aligned}
& -\frac{1+\gamma_{\mathbf{z}} D_{\mathbf{z y}}}{D_{\mathbf{z y}} D_{\mathbf{u v}}\left(1+\gamma_{\mathbf{z}}\right) \gamma_{\mathbf{u}}}+\frac{1+\gamma_{\mathbf{v}} D_{\mathbf{v w}}}{D_{\mathbf{z y}} D_{\mathbf{u x}} \gamma_{\mathbf{z}} \gamma_{\mathbf{u}}\left(1+\gamma_{\mathbf{v}}\right)} \\
& =\frac{-\left(1+\gamma_{\mathbf{z}} D_{\mathbf{z y}}\right) D_{\mathbf{u x}} D_{\mathbf{v w}} \gamma_{\mathbf{z}}\left(1+\gamma_{\mathbf{v}}\right)+\left(1+\gamma_{\mathbf{v}} D_{\mathbf{v w}}\right) D_{\mathbf{u v}}\left(1+\gamma_{\mathbf{z}}\right)}{D_{\mathbf{z y}} D_{\mathbf{u x}} D_{\mathbf{u v}} D_{\mathbf{v w}} D \gamma_{\mathbf{z}} \gamma_{\mathbf{u}}\left(1+\gamma_{\mathbf{v}}\right)} \\
& =\frac{\gamma_{\mathbf{v}}}{D D_{\mathbf{u v}}\left(1+\gamma_{\mathbf{v}}\right)}\left\{-D_{\mathbf{u x}} D_{\mathbf{v w}} \gamma_{\mathbf{z}}\left(1+\gamma_{\mathbf{v}}\right)-D_{\mathbf{z y}} D_{\mathbf{u x}} D_{\mathbf{v w}} \gamma_{\mathbf{z}}{ }^{2}\left(1+\gamma_{\mathbf{v}}\right)\right. \\
& \left.+D_{\mathbf{u v}}\left(1+\gamma_{\mathbf{z}}\right)+D_{\mathbf{u v}} D_{\mathbf{v w}} \gamma_{\mathbf{v}}\left(1+\gamma_{\mathbf{z}}\right)\right\} .
\end{aligned}
$$


Using (16) and $D_{\mathbf{z y}} D_{\mathbf{u x}} D_{\mathbf{v w}} \gamma_{\mathbf{z}}=\frac{1}{\gamma_{\mathbf{u}} \gamma_{\mathbf{v}}}$, then we have

$$
\begin{aligned}
& =\frac{\gamma_{\mathbf{v}}}{D D_{\mathbf{u v}}\left(1+\gamma_{\mathbf{v}}\right)}\left\{-\left(\gamma_{\mathbf{z}} \gamma_{\mathbf{v}} D_{\mathbf{u v}} D_{\mathbf{v w}}+\gamma_{\mathbf{z}} D_{\mathbf{u v}}+\gamma_{\mathbf{z}} D_{\mathbf{v w}}+\gamma_{\mathbf{z}} D_{\mathbf{u w}}+\frac{\gamma_{\mathbf{z}}}{\gamma_{\mathbf{v}}} D_{\mathbf{u w}}\right.\right. \\
& \left.\left.-\frac{\gamma_{\mathbf{z}}}{\gamma_{\mathbf{v}}}\left(2 \gamma_{\mathbf{v}}+1\right)\right)-\frac{\gamma_{\mathbf{z}}\left(1+\gamma_{\mathbf{v}}\right)}{\gamma_{\mathbf{u}} \gamma_{\mathbf{v}}}+D_{\mathbf{u v}}\left(1+\gamma_{\mathbf{z}}\right)+D_{\mathbf{u v}} D_{\mathbf{v w}} \gamma_{\mathbf{v}}\left(1+\gamma_{\mathbf{z}}\right)\right\} \\
& =\frac{1}{D D_{\mathbf{u v}}\left(1+\gamma_{\mathbf{v}}\right)}\left\{\gamma_{\mathbf{v}}^{2}\left(1+\frac{(\mathbf{u} \cdot \mathbf{v})}{s^{2}}+\frac{(\mathbf{v} \cdot \mathbf{w})}{s^{2}}+\frac{1}{s^{4}}(\mathbf{u} \cdot \mathbf{v})(\mathbf{v} \cdot \mathbf{w})\right)\right. \\
& -\gamma_{\mathbf{u}} \gamma_{\mathbf{v}}^{2}\left(1+\frac{(\mathbf{u} \cdot \mathbf{v})}{s^{2}}+\frac{(\mathbf{v} \cdot \mathbf{w})}{s^{2}}+\frac{1}{s^{4}}(\mathbf{u} \cdot \mathbf{v})(\mathbf{v} \cdot \mathbf{w})\right) \\
& -\gamma_{\mathbf{u}} \gamma_{\mathbf{v}}^{2}\left(1+\frac{(\mathbf{u} \cdot \mathbf{v})}{s^{2}}+\frac{(\mathbf{u} \cdot \mathbf{w})}{s^{2}}+\frac{1}{s^{4}}(\mathbf{u} \cdot \mathbf{v})(\mathbf{u} \cdot \mathbf{w})\right) \\
& -\gamma_{\mathbf{u}} \gamma_{\mathbf{v}}\left(1+\frac{(\mathbf{u} \cdot \mathbf{v})}{s^{2}}+\frac{(\mathbf{u} \cdot \mathbf{w})}{s^{2}}+\frac{1}{s^{4}}(\mathbf{u} \cdot \mathbf{v})(\mathbf{u} \cdot \mathbf{w})\right) \\
& \left.+\gamma_{\mathbf{u}} \gamma_{\mathbf{v}}\left(2 \gamma_{\mathbf{v}}+1\right)\left(1+\frac{(\mathbf{u} \cdot \mathbf{v})}{s^{2}}\right)-\gamma_{\mathbf{v}}{ }^{2}\left(1+\frac{(\mathbf{u} \cdot \mathbf{v})}{s^{2}}\right)\right\} \\
& =-\frac{\gamma_{\mathbf{v}}}{s^{2} D\left(1+\gamma_{\mathbf{v}}\right)}\left\{\gamma_{\mathbf{u}}\left(\gamma_{\mathbf{v}}+1\right)(\mathbf{u} \cdot \mathbf{w})+\left(\gamma_{\mathbf{v}}-1\right) \gamma_{\mathbf{u}}(\mathbf{v} \cdot \mathbf{w})\right\} \\
& =\frac{B}{D}
\end{aligned}
$$

Hence $\operatorname{gyr}[\mathbf{u}, \mathbf{v}] \mathbf{w}=\mathbf{w}+\frac{A \mathbf{u}+B \mathbf{v}}{\mathrm{D}}$ holds. By applying the left cancellation law for the Equation (14), we obtain (G3).

We prove (G4). We prove that gyr $[\mathbf{u}, \mathbf{v}]$ is automorphism for every pair $\mathbf{u}, \mathbf{v} \in \mathbb{V}$. To prove (G4), we first show the gyration preserves, the inner product of $\mathbb{V}$ and the norm. So, we compute

$$
\operatorname{gyr}[\mathbf{u}, \mathbf{v}] \mathbf{a} \cdot \operatorname{gyr}[\mathbf{u}, \mathbf{v}] \mathbf{b}=\mathbf{a} \cdot \mathbf{b}
$$

for all $\mathbf{a}, \mathbf{b}, \mathbf{u}, \mathbf{v} \in \mathbb{V}_{s}$. By applying the Equation (15), we have

$$
\operatorname{gyr}[\mathbf{u}, \mathbf{v}] \mathbf{a}=\mathbf{a}+\frac{A_{\mathbf{a}} \mathbf{u}+B_{\mathbf{a}} \mathbf{v}}{D}
$$

and

$$
\operatorname{gyr}[\mathbf{u}, \mathbf{v}] \mathbf{b}=\mathbf{b}+\frac{A_{\mathbf{b}} \mathbf{u}+B_{\mathbf{b}} \mathbf{v}}{D}
$$

respectively, where

$$
\begin{aligned}
A_{\mathbf{a}} & =-\frac{1}{s^{2}} \frac{\gamma_{\mathbf{u}}^{2}}{\gamma_{\mathbf{u}}+1}\left(\gamma_{\mathbf{v}}-1\right)(\mathbf{u} \cdot \mathbf{a})+\frac{1}{s^{2}} \gamma_{\mathbf{u}} \gamma_{\mathbf{v}}(\mathbf{v} \cdot \mathbf{a})+\frac{2}{s^{4}} \frac{\gamma_{\mathbf{u}}^{2} \gamma_{\mathbf{v}}^{2}}{\left(\gamma_{\mathbf{u}}+1\right)\left(\gamma_{\mathbf{v}}+1\right)}(\mathbf{u} \cdot \mathbf{v})(\mathbf{v} \cdot \mathbf{a}), \\
B_{\mathbf{a}} & =-\frac{1}{s^{2}} \frac{\gamma_{\mathbf{v}}}{\gamma_{\mathbf{v}}+1}\left\{\gamma_{\mathbf{u}}\left(\gamma_{\mathbf{v}}+1\right)(\mathbf{u} \cdot \mathbf{a})+\left(\gamma_{\mathbf{u}}-1\right) \gamma_{\mathbf{v}}(\mathbf{v} \cdot \mathbf{a})\right\} .
\end{aligned}
$$

The terms $A_{\mathbf{b}}$ and $B_{\mathbf{b}}$ are defined in the similar way an $A_{\mathbf{a}}$ and $B_{\mathbf{b}}$ respectively. Then we have

$$
\begin{aligned}
& \operatorname{gyr}[\mathbf{u}, \mathbf{v}] \mathbf{a} \cdot \operatorname{gyr}[\mathbf{u}, \mathbf{v}] \mathbf{b}=\mathbf{a} \cdot \mathbf{b}+\frac{A_{\mathbf{a}}(\mathbf{u} \cdot \mathbf{b})+B_{\mathbf{a}}(\mathbf{v} \cdot \mathbf{b})}{D}+\frac{A_{\mathbf{b}}(\mathbf{u} \cdot \mathbf{a})+B_{\mathbf{b}}(\mathbf{v} \cdot \mathbf{a})}{D} \\
& +\frac{1}{D^{2}}\left\{A_{\mathbf{a}} A_{\mathbf{b}}\|\mathbf{u}\|^{2}+A_{\mathbf{a}} B_{\mathbf{b}}(\mathbf{u} \cdot \mathbf{v})+A_{\mathbf{b}} B_{\mathbf{a}}(\mathbf{u} \cdot \mathbf{v})+B_{\mathbf{a}} B_{\mathbf{b}}\|\mathbf{v}\|^{2}\right\}
\end{aligned}
$$


We show that terms other than $\mathbf{a} \cdot \mathbf{b}$ of the right-hand side of the Equation (18) equal to zero.

$$
\begin{aligned}
& A_{\mathbf{a}}(\mathbf{u} \cdot \mathbf{b})+B_{\mathbf{a}}(\mathbf{v} \cdot \mathbf{b}) \\
&=-\frac{1}{s^{2}} \frac{\gamma_{\mathbf{u}}{ }^{2}}{\gamma_{\mathbf{u}}+1}\left(\gamma_{\mathbf{v}}-1\right)(\mathbf{u} \cdot \mathbf{a})(\mathbf{u} \cdot \mathbf{b})+\frac{1}{s^{2}} \gamma_{\mathbf{u}} \gamma_{\mathbf{v}}(\mathbf{v} \cdot \mathbf{a})(\mathbf{u} \cdot \mathbf{b}) \\
&+\frac{2}{s^{4}} \frac{\gamma_{\mathbf{u}}{ }^{2} \gamma_{\mathbf{v}}{ }^{2}}{\left(\gamma_{\mathbf{u}}+1\right)\left(\gamma_{\mathbf{v}}+1\right)}(\mathbf{u} \cdot \mathbf{v})(\mathbf{v} \cdot \mathbf{a})(\mathbf{u} \cdot \mathbf{b}) \\
&-\frac{1}{s^{2}} \gamma_{\mathbf{u}} \gamma_{\mathbf{v}}(\mathbf{u} \cdot \mathbf{a})(\mathbf{v} \cdot \mathbf{b})-\frac{1}{s^{2}} \frac{\gamma_{\mathbf{v}}{ }^{2}}{\gamma_{\mathbf{v}}+1}\left(\gamma_{\mathbf{u}}-1\right)(\mathbf{v} \cdot \mathbf{a})(\mathbf{v} \cdot \mathbf{b}) . \\
& A_{b}(\mathbf{u} \cdot \mathbf{a})+B_{b}(\mathbf{v} \cdot \mathbf{a}) \\
&=-\frac{1}{s^{2}} \frac{\gamma_{\mathbf{u}}{ }^{2}}{\gamma_{\mathbf{u}}+1}\left(\gamma_{\mathbf{v}}-1\right)(\mathbf{u} \cdot \mathbf{a})(\mathbf{u} \cdot \mathbf{b})+\frac{1}{s^{2}} \gamma_{\mathbf{u}} \gamma_{\mathbf{v}}(\mathbf{u} \cdot \mathbf{a})(\mathbf{v} \cdot \mathbf{b}) \\
&+\frac{2}{s^{4}} \frac{\gamma_{\mathbf{u}}{ }^{2} \gamma_{\mathbf{v}}{ }^{2}}{\left(\gamma_{\mathbf{u}}+1\right)\left(\gamma_{\mathbf{v}}+1\right)}(\mathbf{u} \cdot \mathbf{v})(\mathbf{u} \cdot \mathbf{a})(\mathbf{v} \cdot \mathbf{b}) \\
&-\frac{1}{s^{2}} \gamma_{\mathbf{u}} \gamma_{\mathbf{v}}(\mathbf{v} \cdot \mathbf{a})(\mathbf{u} \cdot \mathbf{b})-\frac{1}{s^{2}} \frac{\gamma_{\mathbf{v}}{ }^{2}}{\gamma_{\mathbf{v}}+1}\left(\gamma_{\mathbf{u}}-1\right)(\mathbf{v} \cdot \mathbf{a})(\mathbf{v} \cdot \mathbf{b}) . \\
& A_{\mathbf{a}}(\mathbf{u} \cdot \mathbf{b})+B_{\mathbf{a}}(\mathbf{v} \cdot \mathbf{b})+A_{\mathbf{b}}(\mathbf{u} \cdot \mathbf{a})+B_{\mathbf{b}}(\mathbf{v} \cdot \mathbf{a}) \\
&=-\frac{2}{s^{2}} \frac{\gamma_{\mathbf{u}}{ }^{2}}{\gamma_{\mathbf{u}}+1}\left(\gamma_{\mathbf{v}}-1\right)(\mathbf{u} \cdot \mathbf{a})(\mathbf{u} \cdot \mathbf{b})+\frac{2}{s^{4}} \frac{\gamma_{\mathbf{u}}{ }^{2} \gamma_{\mathbf{v}}{ }^{2}}{\left(\gamma_{\mathbf{u}}+1\right)\left(\gamma_{\mathbf{v}}+1\right)}(\mathbf{u} \cdot \mathbf{v})(\mathbf{u} \cdot \mathbf{a})(\mathbf{v} \cdot \mathbf{b}) \\
&-\frac{2}{s^{2}} \frac{\gamma_{\mathbf{u}}{ }^{2}}{\gamma_{\mathbf{u}}+1}\left(\gamma_{\mathbf{v}}-1\right)(\mathbf{v} \cdot \mathbf{a})(\mathbf{u} \cdot \mathbf{b})+\frac{2}{s^{4}} \frac{\gamma_{\mathbf{u}}{ }^{2} \gamma_{\mathbf{v}}{ }^{2}}{\left(\gamma_{\mathbf{u}}+1\right)\left(\gamma_{\mathbf{v}}+1\right)}(\mathbf{u} \cdot \mathbf{v})(\mathbf{v} \cdot \mathbf{a})(\mathbf{v} \cdot \mathbf{b}) .
\end{aligned}
$$

Then we compute each terms of the sum

$$
\begin{aligned}
A_{\mathbf{a}} A_{\mathbf{b}}\|\mathbf{u}\|^{2} & +A_{\mathbf{a}} B_{\mathbf{b}}(\mathbf{u} \cdot \mathbf{v})+A_{\mathbf{b}} B_{\mathbf{a}}(\mathbf{u} \cdot \mathbf{v})+B_{\mathbf{a}} B_{\mathbf{b}}\|\mathbf{v}\|^{2} \\
& A_{\mathbf{a}} A_{\mathbf{b}}\|\mathbf{u}\|^{2} \\
& =\|\mathbf{u}\|^{2}\left\{\frac{1}{s^{4}} \frac{\gamma_{\mathbf{u}}{ }^{4}}{\left(\gamma_{\mathbf{u}}+1\right)^{2}}\left(\gamma_{\mathbf{v}}-1\right)^{2}(\mathbf{u} \cdot \mathbf{a})(\mathbf{u} \cdot \mathbf{b})-\frac{1}{s^{4}} \frac{\gamma_{\mathbf{u}}{ }^{2}}{\gamma_{\mathbf{u}}+1}\left(\gamma_{\mathbf{v}}-1\right) \gamma_{\mathbf{u}} \gamma_{\mathbf{v}}(\mathbf{u} \cdot \mathbf{a})(\mathbf{v} \cdot \mathbf{b})\right. \\
& -\frac{2}{s^{6}} \frac{\gamma_{\mathbf{u}}{ }^{2}}{\gamma_{\mathbf{u}}+1}\left(\gamma_{\mathbf{v}}-1\right) \frac{\gamma_{\mathbf{u}}{ }^{2} \gamma_{\mathbf{v}}{ }^{2}}{\left(\gamma_{\mathbf{u}}+1\right)\left(\gamma_{\mathbf{v}}+1\right)}(\mathbf{u} \cdot \mathbf{v})(\mathbf{u} \cdot \mathbf{a})(\mathbf{u} \cdot \mathbf{b}) \\
& -\frac{1}{s^{4}} \gamma_{\mathbf{u}} \gamma_{\mathbf{v}} \frac{\gamma_{\mathbf{u}}{ }^{2}}{\gamma_{\mathbf{u}}+1}\left(\gamma_{\mathbf{v}}-1\right)(\mathbf{v} \cdot \mathbf{a})(\mathbf{u} \cdot \mathbf{b})+\frac{1}{s^{4}} \gamma_{\mathbf{u}}{ }^{2} \gamma_{\mathbf{v}}{ }^{2}(\mathbf{v} \cdot \mathbf{a})(\mathbf{v} \cdot \mathbf{b}) \\
& +\frac{2}{s^{6}} \gamma_{\mathbf{u}} \gamma_{\mathbf{v}} \frac{\gamma_{\mathbf{u}}{ }^{2} \gamma_{\mathbf{v}}{ }^{2}}{\left(\gamma_{\mathbf{u}}+1\right)\left(\gamma_{\mathbf{v}}+1\right)}(\mathbf{u} \cdot \mathbf{v})(\mathbf{v} \cdot \mathbf{a})(\mathbf{v} \cdot \mathbf{b}) \\
& -\frac{2}{s^{6}} \frac{\gamma_{\mathbf{u}}{ }^{2} \gamma_{\mathbf{v}}{ }^{2}}{\left(\gamma_{\mathbf{u}}+1\right)\left(\gamma_{\mathbf{v}}+1\right)} \frac{\gamma_{\mathbf{u}}{ }^{2}}{\gamma_{\mathbf{u}}+1}\left(\gamma_{\mathbf{v}}-1\right)(\mathbf{u} \cdot \mathbf{v})(\mathbf{v} \cdot \mathbf{a})(\mathbf{u} \cdot \mathbf{b}) \\
& +\frac{2}{s^{6}} \frac{\gamma_{\mathbf{u}}{ }^{2} \gamma_{\mathbf{v}}{ }^{2}}{\left(\gamma_{\mathbf{u}}+1\right)\left(\gamma_{\mathbf{v}}+1\right)} \gamma_{\mathbf{u}} \gamma_{\mathbf{v}}(\mathbf{u} \cdot \mathbf{v})(\mathbf{v} \cdot \mathbf{a})(\mathbf{v} \cdot \mathbf{b}) \\
& \left.+\frac{4}{s^{8}} \frac{\gamma_{\mathbf{u}}{ }^{4} \gamma_{\mathbf{v}}{ }^{4}}{\left(\gamma_{\mathbf{u}}+1\right)^{2}\left(\gamma_{\mathbf{v}}+1\right)^{2}}(\mathbf{u} \cdot \mathbf{v})^{2}(\mathbf{v} \cdot \mathbf{a})(\mathbf{v} \cdot \mathbf{b})\right\}
\end{aligned}
$$


By $\frac{\|\mathbf{u}\|^{2}}{s^{2}}=\frac{\gamma_{\mathbf{u}^{2}-1}}{\gamma \mathbf{u}^{2}}$ this equation is rewritten in the following.

$$
\begin{aligned}
& A_{\mathbf{a}} A_{\mathbf{b}}\|\mathbf{u}\|^{2} \\
= & \frac{1}{s^{2}} \frac{\gamma_{\mathbf{u}}{ }^{2}}{\left(\gamma_{\mathbf{u}}+1\right)}\left(\gamma_{\mathbf{v}}-1\right)^{2}\left(\gamma_{\mathbf{u}}-1\right)(\mathbf{u} \cdot \mathbf{a})(\mathbf{u} \cdot \mathbf{b})-\frac{1}{s^{2}} \gamma_{\mathbf{u}} \gamma_{\mathbf{v}}\left(\gamma_{\mathbf{u}}-1\right)\left(\gamma_{\mathbf{v}}-1\right)(\mathbf{u} \cdot \mathbf{a})(\mathbf{v} \cdot \mathbf{b}) \\
& -\frac{2}{s^{4}} \frac{\gamma_{\mathbf{u}}{ }^{2} \gamma_{\mathbf{v}}{ }^{2}}{\left(\gamma_{\mathbf{u}}+1\right)\left(\gamma_{\mathbf{v}}+1\right)}\left(\gamma_{\mathbf{u}}-1\right)\left(\gamma_{\mathbf{v}}-1\right)(\mathbf{u} \cdot \mathbf{v})(\mathbf{u} \cdot \mathbf{a})(\mathbf{u} \cdot \mathbf{b}) \\
& -\frac{1}{s^{2}} \gamma_{\mathbf{u}} \gamma_{\mathbf{v}}\left(\gamma_{\mathbf{u}}-1\right)\left(\gamma_{\mathbf{v}}-1\right)(\mathbf{v} \cdot \mathbf{a})(\mathbf{u} \cdot \mathbf{b})+\frac{1}{s^{2}} \gamma_{\mathbf{v}}{ }^{2}\left(\gamma_{\mathbf{u}}{ }^{2}-1\right)(\mathbf{v} \cdot \mathbf{a})(\mathbf{v} \cdot \mathbf{b}) \\
& +\frac{4}{s^{4}} \frac{\gamma_{\mathbf{u}} \gamma_{\mathbf{v}}{ }^{3}}{\left(\gamma_{\mathbf{v}}+1\right)}\left(\gamma_{\mathbf{u}}-1\right)(\mathbf{u} \cdot \mathbf{v})(\mathbf{v} \cdot \mathbf{a})(\mathbf{v} \cdot \mathbf{b}) \\
& \left.-\frac{2}{s^{4}} \frac{\gamma_{\mathbf{u}}{ }^{2} \gamma_{\mathbf{v}}{ }^{2}}{\left(\gamma_{\mathbf{u}}+1\right)\left(\gamma_{\mathbf{v}}+1\right)}\left(\gamma_{\mathbf{u}}-1\right)\left(\gamma_{\mathbf{v}}-1\right)(\mathbf{u} \cdot \mathbf{v})(\mathbf{v} \cdot \mathbf{a})(\mathbf{u} \cdot \mathbf{b})\right) \\
& +\frac{4}{s^{6}} \frac{\gamma_{\mathbf{u}}{ }^{2} \gamma_{\mathbf{v}}}{\left(\gamma_{\mathbf{u}}+1\right)\left(\gamma_{\mathbf{v}}+1\right)^{2}}\left(\gamma_{\mathbf{u}}-1\right)(\mathbf{u} \cdot \mathbf{v})^{2}(\mathbf{v} \cdot \mathbf{a})(\mathbf{v} \cdot \mathbf{b}) .
\end{aligned}
$$

Then we obtain

$$
\begin{aligned}
A_{\mathbf{a}} & B_{\mathbf{b}}(\mathbf{u} \cdot \mathbf{v}) \\
= & \frac{1}{s^{4}} \frac{\gamma_{\mathbf{u}}{ }^{3} \gamma_{\mathbf{v}}}{\left(\gamma_{\mathbf{u}}+1\right)^{2}}\left(\gamma_{\mathbf{v}}-1\right)(\mathbf{u} \cdot \mathbf{v})(\mathbf{u} \cdot \mathbf{a})(\mathbf{u} \cdot \mathbf{b}) \\
& +\frac{1}{s^{4}} \frac{\gamma_{\mathbf{u}}{ }^{2} \gamma_{\mathbf{v}}{ }^{2}}{\left(\gamma_{\mathbf{u}}+1\right)\left(\gamma_{\mathbf{v}}-1\right)}\left(\gamma_{\mathbf{u}}-1\right)\left(\gamma_{\mathbf{v}}-1\right)(\mathbf{u} \cdot \mathbf{v})(\mathbf{u} \cdot \mathbf{a})(\mathbf{v} \cdot \mathbf{b}) \\
& -\frac{1}{s^{4}} \gamma_{\mathbf{u}}{ }^{2} \gamma_{\mathbf{v}}{ }^{2}(\mathbf{u} \cdot \mathbf{v})(\mathbf{v} \cdot \mathbf{a})(\mathbf{u} \cdot \mathbf{b})-\frac{1}{s^{4}} \frac{\gamma_{\mathbf{u}} \gamma_{\mathbf{v}}{ }^{3}}{\left(\gamma_{\mathbf{v}}+1\right)}\left(\gamma_{\mathbf{u}}-1\right)(\mathbf{u} \cdot \mathbf{v})(\mathbf{v} \cdot \mathbf{a})(\mathbf{v} \cdot \mathbf{b}) \\
& -\frac{2}{s^{6}} \frac{\gamma_{\mathbf{u}}{ }^{3} \gamma_{\mathbf{v}}{ }^{3}}{\left(\gamma_{\mathbf{u}}+1\right)\left(\gamma_{\mathbf{v}}+1\right)}(\mathbf{u} \cdot \mathbf{v})^{2}(\mathbf{v} \cdot \mathbf{a})(\mathbf{u} \cdot \mathbf{b}) \\
& -\frac{2}{s^{6}} \frac{\gamma_{\mathbf{u}}{ }^{2} \gamma_{\mathbf{v}}{ }^{4}}{\left(\gamma_{\mathbf{u}}+1\right)\left(\gamma_{\mathbf{v}}+1\right)^{2}}\left(\gamma_{\mathbf{u}}-1\right)(\mathbf{u} \cdot \mathbf{v})^{2}(\mathbf{v} \cdot \mathbf{a})(\mathbf{v} \cdot \mathbf{b}) . \\
& A_{b} B_{a}(\mathbf{u} \cdot \mathbf{v}) \\
= & \frac{1}{s^{4}} \frac{\gamma_{\mathbf{u}}{ }^{3} \gamma_{\mathbf{v}}}{\left(\gamma_{\mathbf{u}}+1\right)^{2}}\left(\gamma_{\mathbf{v}}-1\right)(\mathbf{u} \cdot \mathbf{v})(\mathbf{u} \cdot \mathbf{a})(\mathbf{u} \cdot \mathbf{b}) \\
& +\frac{1}{s^{4}} \frac{\gamma_{\mathbf{u}}{ }^{2} \gamma_{\mathbf{v}}{ }^{2}}{\left(\gamma_{\mathbf{u}}+1\right)\left(\gamma_{\mathbf{v}}-1\right)}\left(\gamma_{\mathbf{u}}-1\right)\left(\gamma_{\mathbf{v}}-1\right)(\mathbf{u} \cdot \mathbf{v})(\mathbf{u} \cdot \mathbf{b})(\mathbf{v} \cdot \mathbf{a}) \\
& -\frac{1}{s^{4}} \gamma_{\mathbf{u}}{ }^{2} \gamma_{\mathbf{v}}{ }^{2}(\mathbf{u} \cdot \mathbf{v})(\mathbf{v} \cdot \mathbf{b})(\mathbf{u} \cdot \mathbf{a})-\frac{1}{s^{4}} \frac{\gamma_{\mathbf{u}} \gamma_{\mathbf{v}}{ }^{3}}{\left(\gamma_{\mathbf{v}}+1\right)}\left(\gamma_{\mathbf{u}}-1\right)(\mathbf{u} \cdot \mathbf{v})(\mathbf{v} \cdot \mathbf{a})(\mathbf{v} \cdot \mathbf{b}) \\
& -\frac{2}{s^{6}} \frac{\gamma_{\mathbf{u}}{ }^{3} \gamma_{\mathbf{v}}{ }^{3}}{\left(\gamma_{\mathbf{u}}+1\right)\left(\gamma_{\mathbf{v}}+1\right)}(\mathbf{u} \cdot \mathbf{v})^{2}(\mathbf{v} \cdot \mathbf{b})(\mathbf{u} \cdot \mathbf{a}) \\
& -\frac{2}{s^{6}} \frac{\gamma_{\mathbf{u}}{ }^{2} \gamma_{\mathbf{v}}{ }^{4}}{\left(\gamma_{\mathbf{u}}+1\right)\left(\gamma_{\mathbf{v}}+1\right)^{2}}\left(\gamma_{\mathbf{u}}-1\right)(\mathbf{u} \cdot \mathbf{v})^{2}(\mathbf{v} \cdot \mathbf{a})(\mathbf{v} \cdot \mathbf{b}) .
\end{aligned}
$$


Calculating $B_{\mathbf{a}} B_{\mathbf{b}}\|\mathbf{v}\|^{2}$ in a way similar to the calculation of $A_{\mathbf{a}} A_{\mathbf{b}}\|\mathbf{u}\|^{2}$, we have

$$
\begin{aligned}
& B_{\mathbf{a}} B_{\mathbf{b}}\|\mathbf{v}\|^{2} \\
= & \frac{1}{s^{2}} \gamma_{\mathbf{u}}^{2}\left(\gamma_{\mathbf{v}}^{2}-1\right)(\mathbf{u} \cdot \mathbf{a})(\mathbf{u} \cdot \mathbf{b})+\frac{1}{s^{2}} \gamma_{\mathbf{u}} \gamma_{\mathbf{v}}\left(\gamma_{\mathbf{u}}-1\right)\left(\gamma_{\mathbf{v}}-1\right)(\mathbf{u} \cdot \mathbf{a})(\mathbf{v} \cdot \mathbf{b}) \\
& +\frac{1}{s^{2}} \gamma_{\mathbf{u}} \gamma_{\mathbf{v}}\left(\gamma_{\mathbf{u}}-1\right)\left(\gamma_{\mathbf{v}}-1\right)(\mathbf{v} \cdot \mathbf{a})(\mathbf{u} \cdot \mathbf{b}) \\
& +\frac{1}{s^{2}} \frac{\gamma_{\mathbf{v}}{ }^{2}}{\gamma_{\mathbf{v}}+1}\left(\gamma_{\mathbf{u}}-1\right)^{2}\left(\gamma_{\mathbf{v}}-1\right)(\mathbf{v} \cdot \mathbf{a})(\mathbf{v} \cdot \mathbf{b}) .
\end{aligned}
$$

Hence, comparing the Equations (19) with (22) we have

$$
\begin{aligned}
& A_{\mathbf{a}} A_{\mathbf{b}}\|\mathbf{u}\|^{2}+A_{\mathbf{a}} B_{\mathbf{b}}(\mathbf{u} \cdot \mathbf{v})+A_{\mathbf{b}} B_{\mathbf{a}}(\mathbf{u} \cdot \mathbf{v})+B_{\mathbf{a}} B_{\mathbf{b}}\|\mathbf{v}\|^{2} \\
= & \frac{1}{s^{2}} \frac{\gamma_{\mathbf{u}}{ }^{2}}{\gamma_{\mathbf{u}}+1}\left(\gamma_{\mathbf{v}}-1\right)(\mathbf{u} \cdot \mathbf{a})(\mathbf{u} \cdot \mathbf{b})\left\{\left(\gamma_{\mathbf{u}}+1\right)\left(\gamma_{\mathbf{v}}+1\right)\right. \\
& \left.+\left(\gamma_{\mathbf{u}}-1\right)\left(\gamma_{\mathbf{v}}-1\right)+2 \gamma_{\mathbf{u}} \gamma_{\mathbf{v}} \frac{\mathbf{u} \cdot \mathbf{v}}{s^{2}}\right\} \\
& -\frac{1}{s^{4}} \frac{\gamma_{\mathbf{u}}^{2} \gamma_{\mathbf{v}}{ }^{2}}{\left(\gamma_{\mathbf{u}}+1\right)\left(\gamma_{\mathbf{v}}+1\right)}(\mathbf{u} \cdot \mathbf{v})(\mathbf{u} \cdot \mathbf{a})(\mathbf{v} \cdot \mathbf{b})\left\{\left(\gamma_{\mathbf{u}}+1\right)\left(\gamma_{\mathbf{v}}+1\right)\right. \\
& \left.+\left(\gamma_{\mathbf{u}}-1\right)\left(\gamma_{\mathbf{v}}-1\right)+2 \gamma_{\mathbf{u}} \gamma_{\mathbf{v}} \frac{\mathbf{u} \cdot \mathbf{v}}{s^{2}}\right\} \\
& +\frac{1}{s^{2}} \frac{\gamma_{\mathbf{u}}^{2}}{\gamma_{\mathbf{u}}+1}\left(\gamma_{\mathbf{v}}-1\right)(\mathbf{v} \cdot \mathbf{a})(\mathbf{u} \cdot \mathbf{b})\left\{\left(\gamma_{\mathbf{u}}+1\right)\left(\gamma_{\mathbf{v}}+1\right)\right. \\
& \left.+\left(\gamma_{\mathbf{u}}-1\right)\left(\gamma_{\mathbf{v}}-1\right)+2 \gamma_{\mathbf{u}} \gamma_{\mathbf{v}} \frac{\mathbf{u} \cdot \mathbf{v}}{s^{2}}\right\} \\
& -\frac{1}{s^{4}} \frac{\gamma_{\mathbf{u}}{ }^{2} \gamma_{\mathbf{v}}{ }^{2}}{\left(\gamma_{\mathbf{u}}+1\right)\left(\gamma_{\mathbf{v}}+1\right)}(\mathbf{u} \cdot \mathbf{v})(\mathbf{v} \cdot \mathbf{a})\left(\mathbf { v } \cdot \mathbf { b } \left\{\left(\gamma_{\mathbf{u}}+1\right)\left(\gamma_{\mathbf{v}}+1\right)\right.\right. \\
& \left.+\left(\gamma_{\mathbf{u}}-1\right)\left(\gamma_{\mathbf{v}}-1\right)+2 \gamma_{\mathbf{u}} \gamma_{\mathbf{v}} \frac{\mathbf{u} \cdot \mathbf{v}}{s^{2}}\right\} \\
= & -D\left(A_{\mathbf{a}}(\mathbf{u} \cdot \mathbf{b})+B_{\mathbf{a}}(\mathbf{v} \cdot \mathbf{b})+A_{\mathbf{b}}(\mathbf{u} \cdot \mathbf{a})+B_{\mathbf{b}}(\mathbf{v} \cdot \mathbf{a})\right) .
\end{aligned}
$$

We conclude that $\operatorname{gyr}[\mathbf{u}, \mathbf{v}] \mathbf{a} \cdot \operatorname{gyr}[\mathbf{u}, \mathbf{v}] \mathbf{b}=\mathbf{a} \cdot \mathbf{b}$.

To prove that $\operatorname{gyr}[\mathbf{u}, \mathbf{v}]$ is a homomorphism for all $\mathbf{u}, \mathbf{v} \in \mathbb{V}_{s}$, we show

$$
\operatorname{gyr}[\mathbf{u}, \mathbf{v}]\left(\mathbf{x} \oplus_{E} \mathbf{y}\right)=\operatorname{gyr}[\mathbf{u}, \mathbf{v}] \mathbf{x} \oplus_{E} \operatorname{gyr}[\mathbf{u}, \mathbf{v}] \mathbf{y}
$$

for all $\mathbf{x}, \mathbf{y} \in \mathbb{V}_{s}$. Applying (15) we have

$$
\operatorname{gyr}[\mathbf{u}, \mathbf{v}]\left(\mathbf{x} \oplus_{E} \mathbf{y}\right)=\mathbf{x} \oplus_{E} \mathbf{y}+\frac{1}{D}\left(A_{\mathbf{x} \oplus_{E} \mathbf{y}} \mathbf{u}+B_{\mathbf{x} \oplus_{E} \mathbf{y}} \mathbf{v}\right) .
$$

Put

$$
\mathbf{x} \oplus_{E} \mathbf{y}=\frac{1+\gamma_{\mathbf{x}} D_{\mathbf{x y}}}{D_{\mathbf{x y}}\left(1+\gamma_{\mathbf{x}}\right)} \mathbf{x}+\frac{1}{\gamma_{\mathbf{x}} D_{\mathbf{x y}}} \mathbf{y}=E_{\mathbf{x y}} \mathbf{x}+F_{\mathbf{x y}} \mathbf{y} .
$$

By a simple calculation we infer that

$$
\begin{aligned}
A_{\mathbf{x} \oplus_{E} \mathbf{y}} & =E_{\mathbf{x y}} A_{\mathbf{x}}+F_{\mathbf{x y}} A_{\mathbf{y}} \\
B_{\mathbf{x} \oplus_{E} \mathbf{y}} & =E_{\mathbf{x y}} B_{\mathbf{x}}+F_{\mathbf{x y}} B_{\mathbf{y}} .
\end{aligned}
$$


We have

$$
\begin{aligned}
& \operatorname{gyr}[\mathbf{u}, \mathbf{v}]\left(\mathbf{x} \oplus_{E} \mathbf{y}\right) \\
& \left.=E_{\mathbf{x y}} \mathbf{x}+F_{\mathbf{x y}} \mathbf{y}+\frac{1}{D}\left\{\left(E_{\mathbf{x y}} A_{\mathbf{x}}+F_{\mathbf{x y}} A_{\mathbf{y}}\right) \mathbf{u}+\left(E_{\mathbf{x y}} B_{\mathbf{x}}+F_{\mathbf{x y}} B_{\mathbf{y}}\right) \mathbf{v}\right)\right\} \\
& =E_{\mathbf{x y}}\left\{\mathbf{x}+\frac{1}{D}\left(A_{\mathbf{x}} \mathbf{u}+B_{\mathbf{x}} \mathbf{v}\right)\right\}+F_{\mathbf{x y}}\left\{\mathbf{y}+\frac{1}{D}\left(A_{\mathbf{y}} \mathbf{u}+B_{\mathbf{y}} \mathbf{v}\right)\right\} \\
& =E_{\mathbf{x y}} g y r[\mathbf{u}, \mathbf{v}] \mathbf{x}+F_{\mathbf{x y}} g \mathbf{g y r}[\mathbf{u}, \mathbf{v}] \mathbf{y} .
\end{aligned}
$$

Then the right-hand side of the Equation (24) is rewritten as the following equation.

$$
\begin{aligned}
& \operatorname{gyr}[\mathbf{u}, \mathbf{v}] \mathbf{x} \oplus_{E} \operatorname{gyr}[\mathbf{u}, \mathbf{v}] \mathbf{y}
\end{aligned}
$$

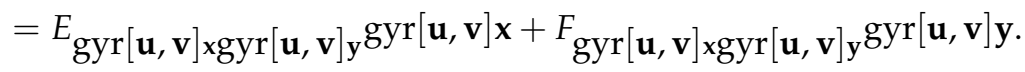

Since gyr $[\mathbf{u}, \mathbf{v}]$ preserves the inner product and the norm of $\mathbb{V}$, we have

$$
\begin{aligned}
& \gamma_{\operatorname{gyr}[\mathbf{u}, \mathbf{v}]_{\mathbf{x}}}=\gamma_{\mathbf{x}} \\
& D_{\operatorname{gyr}[\mathbf{u}, \mathbf{v}]_{\mathbf{x g y r}}[\mathbf{u}, \mathbf{v}]_{\mathbf{y}}=D_{\mathbf{x y}}}
\end{aligned}
$$

so that

$$
E_{\text {gyr }[\mathbf{u}, \mathbf{v}] \operatorname{xgyr}[\mathbf{u}, \mathbf{v}]_{\mathbf{y}}}=E_{\mathbf{x y}}
$$

and

$$
F_{\text {gyr }[\mathbf{u}, \mathbf{v}] \operatorname{xgyr}[\mathbf{u}, \mathbf{v}] \mathbf{y}}=F_{\mathbf{x y}} .
$$

Hence $\operatorname{gyr}[\mathbf{u}, \mathbf{v}]\left(\mathbf{x} \oplus_{E} \mathbf{y}\right)=\operatorname{gyr}[\mathbf{u}, \mathbf{v}] \mathbf{x} \oplus_{E} \operatorname{gyr}[\mathbf{u}, \mathbf{v}] \mathbf{y}$. We conclude that gyr $[\mathbf{u}, \mathbf{v}]$ is a homomorphism.

We observe that gyr $[\mathbf{u}, \mathbf{v}]$ is bijective for every pair of $\mathbf{u}, \mathbf{v} \in \mathbb{V}_{s}$. To prove this, we compute $\operatorname{gyr}[\mathbf{u}, \mathbf{v}](\operatorname{gyr}[\mathbf{v}, \mathbf{u}] \mathbf{w})=\mathbf{w}$ for every $\mathbf{w} \in \mathbb{V}_{s}$. We denote

$$
\begin{aligned}
A_{\mathbf{a b}}(\mathbf{c}) & =-\frac{1}{s^{2}} \frac{\gamma_{\mathbf{a}}{ }^{2}}{\gamma_{\mathbf{a}}+1}\left(\gamma_{\mathbf{b}}-1\right)(\mathbf{a} \cdot \mathbf{c})+\frac{1}{s^{2}} \gamma_{\mathbf{a}} \gamma_{\mathbf{b}}(\mathbf{b} \cdot \mathbf{c})+\frac{2}{s^{4}} \frac{\gamma_{\mathbf{a}}{ }^{2} \gamma_{\mathbf{b}}{ }^{2}}{\left(\gamma_{\mathbf{a}}+1\right)\left(\gamma_{\mathbf{b}}+1\right)}(\mathbf{a} \cdot \mathbf{b})(\mathbf{b} \cdot \mathbf{c}) \\
B_{\mathbf{a b}}(\mathbf{c}) & =-\frac{1}{s^{2}} \frac{\gamma_{\mathbf{b}}}{\gamma_{\mathbf{b}}+1}\left\{\gamma_{\mathbf{a}}\left(\gamma_{\mathbf{b}}+1\right)(\mathbf{a} \cdot \mathbf{c})+\left(\gamma_{\mathbf{a}}-1\right) \gamma_{\mathbf{b}}(\mathbf{b} \cdot \mathbf{c})\right\},
\end{aligned}
$$

where $\mathbf{a}, \mathbf{b}, \mathbf{c} \in \mathbb{V}_{s}$. Then applying (15), we have

$$
\begin{aligned}
& \operatorname{gyr}[\mathbf{u}, \mathbf{v}](\operatorname{gyr}[\mathbf{v}, \mathbf{u}] \mathbf{w}) \\
= & \operatorname{gyr}[\mathbf{u}, \mathbf{v}]\left(\mathbf{w}+\frac{A_{\mathbf{v u}}(\mathbf{w}) \mathbf{v}+B_{\mathbf{v u}}(\mathbf{w}) \mathbf{u}}{D}\right) \\
= & \mathbf{w}+\frac{A_{\mathbf{v u}}(\mathbf{w}) \mathbf{v}+B_{\mathbf{v u}}(\mathbf{w}) \mathbf{u}}{D} \\
& +\frac{1}{D}\left\{A_{\mathbf{u v}}\left(\mathbf{w}+\frac{A_{\mathbf{v u}}(\mathbf{w}) \mathbf{v}+B_{\mathbf{v u}}(\mathbf{w}) \mathbf{u}}{D}\right) \mathbf{u}+B_{\mathbf{u v}}\left(\mathbf{w}+\frac{A_{\mathbf{v u}}(\mathbf{w}) \mathbf{v}+B_{\mathbf{v u}}(\mathbf{w}) \mathbf{u}}{D}\right) \mathbf{v}\right\} .
\end{aligned}
$$

We compute

$$
\begin{aligned}
& A_{\mathbf{u v}}\left(\mathbf{w}+\frac{A_{\mathbf{v u}}(\mathbf{w}) \mathbf{v}+B_{\mathbf{v u}}(\mathbf{w}) \mathbf{u}}{D}\right) \\
= & -\frac{1}{s^{2}} \frac{\gamma_{\mathbf{u}}{ }^{2}}{\gamma_{\mathbf{u}}+1}\left(\gamma_{\mathbf{v}}-1\right)\left(\mathbf{u} \cdot\left(\mathbf{w}+\frac{A_{\mathbf{v u}}(\mathbf{w}) \mathbf{v}+B_{\mathbf{v u}}(\mathbf{w}) \mathbf{u}}{D}\right)\right) \\
& +\frac{1}{s^{2}} \gamma_{\mathbf{u}} \gamma_{\mathbf{v}}\left(\mathbf{v} \cdot\left(\mathbf{w}+\frac{A_{\mathbf{v u}}(\mathbf{w}) \mathbf{v}+B_{\mathbf{v u}}(\mathbf{w}) \mathbf{u}}{D}\right)\right) \\
& +\frac{2}{s^{4}} \frac{\gamma_{\mathbf{u}}{ }^{2} \gamma_{\mathbf{v}}}{\left(\gamma_{\mathbf{u}}+1\right)\left(\gamma_{\mathbf{v}}+1\right)}(\mathbf{u} \cdot \mathbf{v})\left(\mathbf{v} \cdot\left(\mathbf{w}+\frac{A_{\mathbf{v u}}(\mathbf{w}) \mathbf{v}+B_{\mathbf{v u}}(\mathbf{w}) \mathbf{u}}{D}\right)\right) \\
= & A_{\mathbf{u v}}(\mathbf{w})+\frac{1}{D} A_{\mathbf{v u}}(\mathbf{w}) A_{\mathbf{u v}}(\mathbf{v})+\frac{1}{D} B_{\mathbf{v u}}(\mathbf{w}) A_{\mathbf{u v}}(\mathbf{u})
\end{aligned}
$$


and

$$
\begin{aligned}
& B_{\mathbf{u v}}\left(\mathbf{w}+\frac{A_{\mathbf{v u}}(\mathbf{w}) \mathbf{v}+B_{\mathbf{v u}}(\mathbf{w}) \mathbf{u}}{D}\right) \\
= & -\frac{1}{s^{2}} \frac{\gamma_{\mathbf{v}}}{\gamma_{\mathbf{v}}+1}\left\{\gamma_{\mathbf{u}}\left(\gamma_{\mathbf{v}}+1\right)\left(\mathbf{u} \cdot\left(\mathbf{w}+\frac{A_{\mathbf{v u}}(\mathbf{w}) \mathbf{v}+B_{\mathbf{v u}}(\mathbf{w}) \mathbf{u}}{D}\right)\right)\right. \\
& \left.+\left(\gamma_{\mathbf{u}}-1\right) \gamma_{\mathbf{v}}\left(\mathbf{v} \cdot\left(\mathbf{w}+\frac{A_{\mathbf{v u}}(\mathbf{w}) \mathbf{v}+B_{\mathbf{v u}}(\mathbf{w}) \mathbf{u}}{D}\right)\right)\right\} \\
= & B_{\mathbf{u v}}(\mathbf{w})+\frac{1}{D} A_{\mathbf{v u}}(\mathbf{w}) B_{\mathbf{u v}}(\mathbf{v})+\frac{1}{D} B_{\mathbf{v u}}(\mathbf{w}) B_{\mathbf{u v}}(\mathbf{u}) .
\end{aligned}
$$

So, we have

$$
\begin{aligned}
& \operatorname{gyr}[\mathbf{u}, \mathbf{v}](\operatorname{gyr}[\mathbf{v}, \mathbf{u}] \mathbf{w}) \\
= & \mathbf{w}+\left\{\frac{B_{\mathbf{v u}(\mathbf{w})}}{D}+\frac{A_{\mathbf{u v}}(\mathbf{w})}{D}+\frac{1}{D^{2}}\left(A_{\mathbf{v u}}(\mathbf{w}) A_{\mathbf{u v}}(\mathbf{v})+B_{\mathbf{v u}}(\mathbf{w}) A_{\mathbf{u v}}(\mathbf{u})\right)\right\} \mathbf{u} \\
& +\left\{\frac{A_{\mathbf{v u}(\mathbf{w})}}{D}+\frac{B_{\mathbf{u v}}(\mathbf{w})}{D}+\frac{1}{D^{2}}\left(A_{\mathbf{v u}}(\mathbf{w}) B_{\mathbf{u v}}(\mathbf{v})+B_{\mathbf{v u}}(\mathbf{w}) B_{\mathbf{u v}}(\mathbf{u})\right)\right\} \mathbf{v} .
\end{aligned}
$$

We show that the coefficients of $\mathbf{u}$ and $\mathbf{v}$ vanish. We compute the coefficient of $\mathbf{u}$.

$$
B_{\mathbf{v u}}(\mathbf{w})+A_{\mathbf{u v}}(\mathbf{w})=-\frac{2}{s^{2}} \frac{\gamma_{\mathbf{u}}^{2}}{\gamma_{\mathbf{u}}+1}\left(\gamma_{\mathbf{v}}-1\right)(\mathbf{u} \cdot \mathbf{w})+\frac{2}{s^{4}} \frac{\gamma_{\mathbf{u}}^{2} \gamma_{\mathbf{v}}{ }^{2}}{\left(\gamma_{\mathbf{u}}+1\right)\left(\gamma_{\mathbf{v}}+1\right)}(\mathbf{u} \cdot \mathbf{v})(\mathbf{v} \cdot \mathbf{w})
$$

We also have

$$
\begin{aligned}
& A_{\mathbf{v u}}(\mathbf{w}) A_{\mathbf{u v}}(\mathbf{v})+B_{\mathbf{v u}}(\mathbf{w}) A_{\mathbf{u v}}(\mathbf{u}) \\
& =\frac{1}{s^{2}} \frac{\gamma_{\mathbf{v}}^{2}}{\gamma_{\mathbf{v}}+1}\left(\gamma_{\mathbf{u}}-1\right)(\mathbf{v} \cdot \mathbf{w}) \frac{1}{s^{2}} \frac{\gamma_{\mathbf{u}}{ }^{2}}{\gamma_{\mathbf{u}}+1}\left(\gamma_{\mathbf{v}}-1\right)(\mathbf{u} \cdot \mathbf{v})-\frac{1}{s^{2}} \frac{\gamma_{\mathbf{v}}{ }^{2}}{\gamma_{\mathbf{v}}+1}\left(\gamma_{\mathbf{u}}-1\right)(\mathbf{v} \cdot \mathbf{w}) \frac{1}{s^{2}} \gamma_{\mathbf{u}} \gamma_{\mathbf{v}}\|\mathbf{v}\|^{2} \\
& -\frac{1}{s^{2}} \frac{\gamma_{\mathbf{v}}^{2}}{\gamma_{\mathbf{v}}+1}\left(\gamma_{\mathbf{u}}-1\right)(\mathbf{v} \cdot \mathbf{w}) \frac{2}{s^{4}} \frac{\gamma_{\mathbf{u}}^{2} \gamma_{\mathbf{v}}^{2}}{\left(\gamma_{\mathbf{u}}+1\right)\left(\gamma_{\mathbf{v}}+1\right)}(\mathbf{u} \cdot \mathbf{v})\|\mathbf{v}\|^{2} \\
& -\frac{1}{s^{2}} \gamma_{\mathbf{u}} \gamma_{\mathbf{v}}(\mathbf{u} \cdot \mathbf{w}) \frac{\gamma_{\mathbf{u}}{ }^{2}}{\gamma_{\mathbf{u}}+1}\left(\gamma_{\mathbf{v}}-1\right)(\mathbf{u} \cdot \mathbf{v})+\frac{1}{s^{2}} \gamma_{\mathbf{u}} \gamma_{\mathbf{v}}(\mathbf{u} \cdot \mathbf{w}) \frac{1}{s^{2}} \gamma_{\mathbf{u}} \gamma_{\mathbf{v}}\|\mathbf{v}\|^{2} \\
& +\frac{1}{s^{2}} \gamma_{\mathbf{u}} \gamma_{\mathbf{v}}(\mathbf{u} \cdot \mathbf{w}) \frac{2}{s^{4}} \frac{\gamma_{\mathbf{u}}^{2} \gamma_{\mathbf{v}}^{2}}{\left(\gamma_{\mathbf{u}}+1\right)\left(\gamma_{\mathbf{v}}+1\right)}(\mathbf{u} \cdot \mathbf{v})\|\mathbf{v}\|^{2} \\
& -\frac{2}{s^{4}} \frac{\gamma_{\mathbf{u}}^{2} \gamma_{\mathbf{v}}^{2}}{\left(\gamma_{\mathbf{u}}+1\right)\left(\gamma_{\mathbf{v}}+1\right)}(\mathbf{u} \cdot \mathbf{v})(\mathbf{v} \cdot \mathbf{w}) \frac{1}{s^{2}} \frac{\gamma_{\mathbf{u}}^{2}}{\gamma_{\mathbf{u}}+1}\left(\gamma_{\mathbf{v}}-1\right)(\mathbf{u} \cdot \mathbf{v}) \\
& +\frac{2}{s^{4}} \frac{\gamma_{\mathbf{u}}^{2} \gamma_{\mathbf{v}}^{2}}{\left(\gamma_{\mathbf{u}}+1\right)\left(\gamma_{\mathbf{v}}+1\right)}(\mathbf{u} \cdot \mathbf{v})(\mathbf{v} \cdot \mathbf{w}) \frac{1}{s^{2}} \gamma_{\mathbf{u}} \gamma_{\mathbf{v}}\|\mathbf{v}\|^{2} \\
& +\frac{2}{s^{4}} \frac{\gamma_{\mathbf{u}}{ }^{2} \gamma_{\mathbf{v}}{ }^{2}}{\left(\gamma_{\mathbf{u}}+1\right)\left(\gamma_{\mathbf{v}}+1\right)}(\mathbf{u} \cdot \mathbf{v})(\mathbf{v} \cdot \mathbf{w}) \frac{2}{s^{4}} \frac{\gamma_{\mathbf{u}}{ }^{2} \gamma_{\mathbf{v}}{ }^{2}}{\left(\gamma_{\mathbf{u}}+1\right)\left(\gamma_{\mathbf{v}}+1\right)}(\mathbf{u} \cdot \mathbf{v})\|\mathbf{v}\|^{2} \\
& +\frac{1}{s^{2}} \gamma_{\mathbf{u}} \gamma_{\mathbf{v}}(\mathbf{v} \cdot \mathbf{w}) \frac{1}{s^{2}} \frac{\gamma_{\mathbf{u}}^{2}}{\gamma_{\mathbf{u}}+1}\left(\gamma_{\mathbf{v}}-1\right)\|\mathbf{u}\|^{2}-\frac{1}{s^{2}} \gamma_{\mathbf{u}} \gamma_{\mathbf{v}}(\mathbf{v} \cdot \mathbf{w}) \frac{1}{s^{2}} \gamma_{\mathbf{u}} \gamma_{\mathbf{v}}(\mathbf{u} \cdot \mathbf{v}) \\
& -\frac{1}{s^{2}} \gamma_{\mathbf{u}} \gamma_{\mathbf{v}}(\mathbf{v} \cdot \mathbf{w}) \frac{2}{s^{4}} \frac{\gamma_{\mathbf{u}}^{2} \gamma_{\mathbf{v}}^{2}}{\left(\gamma_{\mathbf{u}}+1\right)\left(\gamma_{\mathbf{v}}+1\right)}(\mathbf{u} \cdot \mathbf{v})^{2} \\
& +\frac{1}{s^{2}} \frac{\gamma_{\mathbf{u}}{ }^{2}}{\gamma_{\mathbf{u}}+1}\left(\gamma_{\mathbf{v}}-1\right)(\mathbf{u} \cdot \mathbf{w}) \frac{\gamma_{\mathbf{u}}{ }^{2}}{\gamma_{\mathbf{u}}+1}\left(\gamma_{\mathbf{v}}-1\right)\|\mathbf{u}\|^{2} \\
& -\frac{1}{s^{2}} \frac{\gamma_{\mathbf{u}}^{2}}{\gamma_{\mathbf{u}}+1}\left(\gamma_{\mathbf{v}}-1\right)(\mathbf{u} \cdot \mathbf{w}) \frac{1}{s^{2}} \gamma_{\mathbf{u}} \gamma_{\mathbf{v}}(\mathbf{u} \cdot \mathbf{v}) \\
& -\frac{1}{s^{2}} \frac{\gamma_{\mathbf{u}}^{2}}{\gamma_{\mathbf{u}}+1}\left(\gamma_{\mathbf{v}}-1\right)(\mathbf{u} \cdot \mathbf{w}) \frac{2}{s^{4}} \frac{\gamma_{\mathbf{u}}^{2} \gamma_{\mathbf{v}}^{2}}{\left(\gamma_{\mathbf{u}}+1\right)\left(\gamma_{\mathbf{v}}+1\right)}(\mathbf{u} \cdot \mathbf{v})^{2} .
\end{aligned}
$$


Applying the gamma identity, we have the following.

$$
\begin{aligned}
& A_{\mathbf{v u}}(\mathbf{w}) A_{\mathbf{u v}}(\mathbf{v})+B_{\mathbf{v u}}(\mathbf{w}) A_{\mathbf{u v}}(\mathbf{u}) \\
= & \frac{1}{s^{4}} \frac{\gamma_{\mathbf{u}}^{2} \gamma_{\mathbf{v}}^{2}}{\left(\gamma_{\mathbf{u}}+1\right)\left(\gamma_{\mathbf{v}}+1\right)}(\mathbf{u} \cdot \mathbf{v})(\mathbf{v} \cdot \mathbf{w})\left(-\gamma_{\mathbf{u}} \gamma_{\mathbf{v}}+\gamma_{\mathbf{u}}+\gamma_{\mathbf{v}}-1-2 \gamma_{\mathbf{u}} \gamma_{\mathbf{v}} \frac{(\mathbf{u} \cdot \mathbf{v})}{s^{2}}\right) \\
& -\frac{1}{s^{2}} \gamma_{\mathbf{u}} \gamma_{\mathbf{v}}(\mathbf{v} \cdot \mathbf{w}) \gamma_{\mathbf{u}} \gamma_{\mathbf{v}} \frac{(\mathbf{u} \cdot \mathbf{v})}{s^{2}}+\frac{1}{s^{2}}\left(\gamma_{\mathbf{u}}{ }^{2}\left(\gamma_{\mathbf{v}}{ }^{2}-1\right)+\frac{\gamma_{\mathbf{u}}{ }^{2}\left(\gamma_{\mathbf{u}}-1\right)\left(\gamma_{\mathbf{v}}-1\right)^{2}}{\gamma_{\mathbf{u}}+1}\right) \\
& +\frac{2}{s^{4}} \frac{\gamma_{\mathbf{u}}{ }^{2}}{\gamma_{\mathbf{u}}+1}\left(\gamma_{\mathbf{v}}-1\right) \gamma_{\mathbf{u}} \gamma_{\mathbf{v}}(\mathbf{u} \cdot \mathbf{v})(\mathbf{u} \cdot \mathbf{w}) \\
= & \frac{1}{s^{4}} \frac{\gamma_{\mathbf{u}}{ }^{2} \gamma_{\mathbf{v}}{ }^{2}}{\left(\gamma_{\mathbf{u}}+1\right)\left(\gamma_{\mathbf{v}}+1\right)}(\mathbf{u} \cdot \mathbf{v})(\mathbf{v} \cdot \mathbf{w})\left(-\left(\gamma_{\mathbf{u}}-1\right)\left(\gamma_{\mathbf{v}}-1\right)-\left(\gamma_{\mathbf{u}}+1\right)\left(\gamma_{\mathbf{v}}+1\right)-2 \gamma_{\mathbf{u}} \gamma_{\mathbf{v}} \frac{(\mathbf{u} \cdot \mathbf{v})}{s^{2}}\right) \\
& +\frac{2}{s^{4}} \frac{\gamma_{\mathbf{u}}{ }^{2}}{\gamma_{\mathbf{u}}+1}\left(\gamma_{\mathbf{v}}-1\right)(\mathbf{u} \cdot \mathbf{w})\left(\left(\gamma_{\mathbf{u}} \gamma_{\mathbf{v}}+1\right)+\gamma_{\mathbf{u}} \gamma_{\mathbf{v}} \frac{(\mathbf{u} \cdot \mathbf{v})}{s^{2}}\right) \\
= & -D\left(B_{\mathbf{v u}}(\mathbf{w})+A_{\mathbf{u v}}(\mathbf{w})\right) .
\end{aligned}
$$

So, the coefficient of $\mathbf{u}$ vanishes.

We compute the coefficient of $\mathbf{v}$.

$$
A_{\mathbf{v u}}(\mathbf{w})+B_{\mathbf{u v}}(\mathbf{w})=-\frac{2}{s^{2}} \frac{\gamma_{\mathbf{v}}^{2}}{\gamma_{\mathbf{v}}+1}\left(\gamma_{\mathbf{u}}-1\right)(\mathbf{v} \cdot \mathbf{w})+\frac{2}{s^{4}} \frac{\gamma_{\mathbf{u}}^{2} \gamma_{\mathbf{v}}{ }^{2}}{\left(\gamma_{\mathbf{u}}+1\right)\left(\gamma_{\mathbf{v}}+1\right)}(\mathbf{u} \cdot \mathbf{v})(\mathbf{u} \cdot \mathbf{w})
$$

We also have

$$
\begin{aligned}
& A_{\mathbf{v u}}(\mathbf{w}) B_{\mathbf{u v}}(\mathbf{v})+B_{\mathbf{v u}}(\mathbf{w}) B_{\mathbf{u v}}(\mathbf{u}) \\
&=\frac{1}{s^{2}} \frac{\gamma_{\mathbf{v}}^{2}}{\gamma_{\mathbf{v}}+1}\left(\gamma_{\mathbf{u}}-1\right)(\mathbf{v} \cdot \mathbf{w}) \frac{1}{s^{2}} \gamma_{\mathbf{u}} \gamma_{\mathbf{v}}(\mathbf{u} \cdot \mathbf{v}) \\
&+\frac{1}{s^{2}} \frac{\gamma_{\mathbf{v}}^{2}}{\gamma_{\mathbf{v}}+1}\left(\gamma_{\mathbf{u}}-1\right)(\mathbf{v} \cdot \mathbf{w}) \frac{1}{s^{2}} \frac{\gamma_{\mathbf{v}}^{2}}{\gamma_{\mathbf{v}}+1}\left(\gamma_{\mathbf{u}}-1\right)\|\mathbf{v}\|^{2} \\
&-\frac{1}{s^{2}} \gamma_{\mathbf{u}} \gamma_{\mathbf{v}}(\mathbf{u} \cdot \mathbf{w}) \frac{1}{s^{2}} \gamma_{\mathbf{u}} \gamma_{\mathbf{v}}(\mathbf{u} \cdot \mathbf{v})-\frac{1}{s^{2}} \gamma_{\mathbf{u}} \gamma_{\mathbf{v}}(\mathbf{u} \cdot \mathbf{w}) \frac{1}{s^{2}} \frac{\gamma_{\mathbf{v}}{ }^{2}}{\gamma_{\mathbf{v}}+1}\left(\gamma_{\mathbf{u}}-1\right)\|\mathbf{v}\|^{2} \\
&-\frac{2}{s^{4}} \frac{\gamma_{\mathbf{u}}^{2} \gamma_{\mathbf{v}}{ }^{2}}{\left(\gamma_{\mathbf{u}}+1\right)\left(\gamma_{\mathbf{v}}+1\right)}(\mathbf{u} \cdot \mathbf{v})(\mathbf{u} \cdot \mathbf{w}) \frac{1}{s^{2}} \gamma_{\mathbf{u}} \gamma_{\mathbf{v}}(\mathbf{u} \cdot \mathbf{v}) \\
&-\frac{2}{s^{4}} \frac{\gamma_{\mathbf{u}}{ }^{2} \gamma_{\mathbf{v}}}{\left(\gamma_{\mathbf{u}}+1\right)\left(\gamma_{\mathbf{v}}+1\right)}(\mathbf{u} \cdot \mathbf{v})(\mathbf{u} \cdot \mathbf{w}) \frac{1}{s^{2}} \frac{\gamma_{\mathbf{v}}{ }^{2}}{\gamma_{\mathbf{v}}+1}\left(\gamma_{\mathbf{u}}-1\right)\|\mathbf{v}\|^{2} \\
&+\frac{1}{s^{2}} \gamma_{\mathbf{u}} \gamma_{\mathbf{v}}(\mathbf{v} \cdot \mathbf{w}) \frac{1}{s^{2}} \gamma_{\mathbf{u}} \gamma_{\mathbf{v}}\|\mathbf{u}\|^{2}+\frac{1}{s^{2}} \gamma_{\mathbf{u}} \gamma_{\mathbf{v}}(\mathbf{v} \cdot \mathbf{w}) \frac{1}{s^{2}} \frac{\gamma_{\mathbf{v}}{ }^{2}}{\gamma_{\mathbf{v}}+1}\left(\gamma_{\mathbf{u}}-1\right)(\mathbf{u} \cdot \mathbf{v}) \\
&+\frac{1}{s^{2}} \frac{\gamma_{\mathbf{u}}^{2}}{\gamma_{\mathbf{u}}+1}\left(\gamma_{\mathbf{v}}-1\right)(\mathbf{u} \cdot \mathbf{w}) \frac{1}{s^{2}} \gamma_{\mathbf{u}} \gamma_{\mathbf{v}}\|\mathbf{u}\|^{2} \\
&+\frac{1}{s^{2}} \frac{\gamma_{\mathbf{u}}^{2}}{\gamma_{\mathbf{u}}+1}\left(\gamma_{\mathbf{v}}-1\right)(\mathbf{u} \cdot \mathbf{w}) \frac{1}{s^{2}} \frac{\gamma_{\mathbf{v}}{ }^{2}}{\gamma_{\mathbf{v}}+1}\left(\gamma_{\mathbf{u}}-1\right)(\mathbf{u} \cdot \mathbf{v}) \\
&=\frac{2}{s^{2}} \frac{\gamma_{\mathbf{v}}^{2}}{\gamma_{\mathbf{v}}+1}\left(\gamma_{\mathbf{u}}-1\right)(\mathbf{v} \cdot \mathbf{w})\left\{\gamma_{\mathbf{u}} \gamma_{\mathbf{v}} \frac{(\mathbf{u} \cdot \mathbf{v})}{s^{2}}+\left(\gamma_{\mathbf{u}}-1\right)\left(\gamma_{\mathbf{v}}-1\right)+\left(\gamma_{\mathbf{u}}+1\right)\left(\gamma_{\mathbf{v}}+1\right)\right\} \\
&-\frac{2}{s^{4}} \frac{\gamma_{\mathbf{u}}{ }^{2} \gamma_{\mathbf{v}}{ }^{2}}{\left(\gamma_{\mathbf{u}}+1\right)\left(\gamma_{\mathbf{v}}+1\right)}(\mathbf{u} \cdot \mathbf{v})(\mathbf{u} \cdot \mathbf{w})\left\{\left(\gamma_{\mathbf{u}}+1\right)\left(\gamma_{\mathbf{v}}+1\right)\right. \\
&\left.+\left(\gamma_{\mathbf{u}}-1\right)\left(\gamma_{\mathbf{v}}-1\right)+\gamma_{\mathbf{u}} \gamma_{\mathbf{v}} \frac{(\mathbf{u} \cdot \mathbf{v})}{s^{2}}\right\} \\
&=-D\left(A_{\mathbf{v u}}(\mathbf{w})+B_{\mathbf{u v}}(\mathbf{w})\right) .
\end{aligned}
$$


So, the coefficient of $\mathbf{v}$ vanishes. Thus, $\operatorname{gyr}[\mathbf{u}, \mathbf{v}](\operatorname{gyr}[\mathbf{v}, \mathbf{u}] \mathbf{w})=\mathbf{w}$ holds for every $\mathbf{w} \in \mathbb{V}_{s}$. Changing $\mathbf{u}$ and $\mathbf{v}, \operatorname{gyr}[\mathbf{v}, \mathbf{u}](\operatorname{gyr}[\mathbf{u}, \mathbf{v}] \mathbf{w})=\mathbf{w}$ also holds for every $\mathbf{w} \in \mathbb{V}_{s}$. We conclude that $\operatorname{gyr}[\mathbf{u}, \mathbf{v}]$ is bijective. Thus, gyr $[\mathbf{u}, \mathbf{v}]$ is an automorphism; a proof of (G4) is complete.

To prove (G5) we first observe gyr $\left[\mathbf{u} \oplus_{\mathbf{E}} \mathbf{v}, \mathbf{v}\right]=\operatorname{gyr}[\mathbf{u}, \mathbf{v}]$ for every pair $\mathbf{u}, \mathbf{v} \in \mathbb{V}_{s}$. Let $\mathbf{u}, \mathbf{v}, \mathbf{w} \in \mathbb{V}_{s}$. Applying (15) we have that

$$
\begin{aligned}
\operatorname{gyr}\left[\mathbf{u} \oplus_{\mathbf{E}} \mathbf{v}, \mathbf{v}\right] \mathbf{w} & =\mathbf{w}+\frac{A^{\prime} \mathbf{u} \oplus_{E} \mathbf{v}+B^{\prime} \mathbf{v}}{D^{\prime}} \\
& =\mathbf{w}+\frac{A^{\prime}}{D^{\prime}} \frac{1+\gamma_{\mathbf{u}} D_{\mathbf{u v}}}{D_{\mathbf{u v}}\left(1+\gamma_{\mathbf{u}}\right)} \mathbf{u}+\frac{1}{D^{\prime}}\left(\frac{A^{\prime}}{D_{\mathbf{u v}} \gamma_{\mathbf{u}}}+B^{\prime}\right) \mathbf{v}
\end{aligned}
$$

where

$$
\begin{aligned}
A^{\prime}= & -\frac{1}{s^{2}} \frac{\gamma_{\mathbf{u} \oplus_{\mathbf{E}} \mathbf{v}^{2}}}{\left(\gamma_{\mathbf{u} \oplus_{\mathbf{E}} \mathbf{v}}+1\right)}\left(\gamma_{\mathbf{v}}-1\right)\left(\mathbf{u} \oplus_{E} \mathbf{v} \cdot \mathbf{w}\right)+\frac{1}{s^{2}} \gamma_{\mathbf{u} \oplus_{\mathbf{E}} \mathbf{v}} \gamma_{\mathbf{v}}(\mathbf{v} \cdot \mathbf{w}) \\
& +\frac{2}{s^{4}} \frac{\gamma_{\mathbf{u} \oplus_{\mathbf{E}} \mathbf{v}}{ }^{2} \gamma_{\mathbf{v}}{ }^{2}}{\left(\gamma_{\mathbf{u} \oplus_{\mathbf{E}} \mathbf{v}}+1\right)\left(\gamma_{\mathbf{v}}+1\right)}\left(\mathbf{u} \oplus_{E} \mathbf{v} \cdot \mathbf{v}\right)(\mathbf{v} \cdot \mathbf{w}) \\
B^{\prime}= & -\frac{1}{s^{2}} \frac{\gamma_{\mathbf{v}}}{\gamma_{\mathbf{v}}+1}\left\{\gamma_{\mathbf{u} \oplus_{\mathbf{E}} \mathbf{v}}\left(\gamma_{\mathbf{v}}+1\right)\left(\mathbf{u} \oplus_{E} \mathbf{v} \cdot \mathbf{w}\right)+\left(\gamma_{\mathbf{u} \oplus_{\mathbf{E}} \mathbf{v}}-1\right) \gamma_{\mathbf{v}}(\mathbf{v} \cdot \mathbf{w})\right\} \\
D^{\prime}= & \gamma_{\left(\mathbf{u} \oplus_{\mathbf{E}} \mathbf{v}\right) \oplus_{\mathbf{E}} \mathbf{v}}+1 \\
= & \gamma_{\mathbf{u} \oplus_{\mathbf{E}} \mathbf{v}} \gamma_{\mathbf{v}}\left(1+\frac{\left(\mathbf{u} \oplus_{E} \mathbf{v}\right) \cdot \mathbf{v}}{s^{2}}\right)+1 .
\end{aligned}
$$

We prove that $\operatorname{gyr}\left[\mathbf{u} \oplus_{\mathbf{E}} \mathbf{v}, \mathbf{v}\right] \mathbf{w}=\operatorname{gyr}[\mathbf{u}, \mathbf{v}] \mathbf{w}$ for every $\mathbf{u}, \mathbf{v}, \mathbf{w} \in \mathbb{V}_{s}$. We have

$$
\begin{aligned}
& \left(\mathbf{u} \oplus_{E} \mathbf{v}\right) \cdot \mathbf{w}=\frac{1+\gamma_{\mathbf{u}} D_{\mathbf{u v}}}{D_{\mathbf{u v}}\left(1+\gamma_{\mathbf{u}}\right)}(\mathbf{u} \cdot \mathbf{w})+\frac{1}{D_{\mathbf{u v}} \gamma_{\mathbf{u}}}(\mathbf{v} \cdot \mathbf{w}), \\
& \left(\mathbf{u} \oplus_{E} \mathbf{v}\right) \cdot \mathbf{v}=\frac{1+\gamma_{\mathbf{u}} D_{\mathbf{u v}}}{D_{\mathbf{u v}}\left(1+\gamma_{\mathbf{u}}\right)}(\mathbf{u} \cdot \mathbf{v})+\frac{1}{D_{\mathbf{u v}} \gamma_{\mathbf{u}}}\|\mathbf{v}\|^{2}, \\
& \gamma_{\mathbf{u} \oplus_{\mathbf{E}} \mathbf{v}}+1=D .
\end{aligned}
$$

Then $A^{\prime}$ is computed as in the following.

$$
\begin{aligned}
& A^{\prime}=\frac{1}{\gamma_{\mathbf{u} \oplus_{\mathbf{E}} \mathbf{v}}+1}\left\{-\gamma_{\mathbf{u} \oplus_{\mathbf{E}} \mathbf{v}}{ }^{2}\left(\gamma_{\mathbf{v}}-1\right) \frac{1+\gamma_{\mathbf{u}} D_{\mathbf{u v}}}{D_{\mathbf{u v}}\left(1+\gamma_{\mathbf{u}}\right)} \frac{(\mathbf{u} \cdot \mathbf{w})}{s^{2}}-\gamma_{\mathbf{u} \oplus_{\mathbf{E}} \mathbf{v}}{ }^{2}\left(\gamma_{\mathbf{v}}-1\right) \frac{1}{D_{\mathbf{u v}} \gamma_{\mathbf{u}}} \frac{(\mathbf{v} \cdot \mathbf{w})}{s^{2}}\right. \\
& +\frac{1}{s^{2}} \gamma_{\mathbf{u} \oplus_{\mathbf{E}} \mathbf{v}} \gamma_{\mathbf{v}}\left(\gamma_{\mathbf{u} \oplus_{\mathbf{E}} \mathbf{v}}+1\right)(\mathbf{v} \cdot \mathbf{w})+\frac{2}{s^{4}} \frac{\gamma_{\mathbf{u} \oplus_{\mathbf{E v}}} \gamma_{\mathbf{v}}{ }^{2}}{\left(\gamma_{\mathbf{v}}+1\right)} \frac{1+\gamma_{\mathbf{u}} D_{\mathbf{u v}}}{D_{\mathbf{u v}}\left(1+\gamma_{\mathbf{u}}\right)}(\mathbf{u} \cdot \mathbf{v})(\mathbf{v} \cdot \mathbf{w}) \\
& \left.+\frac{2}{s^{4}} \frac{\gamma_{\mathbf{u} \oplus \mathbf{E v}}{ }^{2} \gamma_{\mathbf{v}}{ }^{2}}{\left(\gamma_{\mathbf{v}}+1\right)} \frac{1}{D_{\mathbf{u v}} \gamma_{\mathbf{u}}}(\mathbf{v} \cdot \mathbf{w})\|\mathbf{v}\|^{2}\right\} \\
& =\frac{1}{D}\left\{-\gamma_{\mathbf{u}}{ }^{2} \gamma_{\mathbf{v}}{ }^{2} D_{\mathbf{u v}}^{2}\left(\gamma_{\mathbf{v}}-1\right) \frac{1+\gamma_{\mathbf{u}} D_{\mathbf{u v}}}{D_{\mathbf{u v}}\left(1+\gamma_{\mathbf{u}}\right)} \frac{(\mathbf{u} \cdot \mathbf{w})}{s^{2}}-\gamma_{\mathbf{u}}{ }^{2} \gamma_{\mathbf{v}}{ }^{2} D_{\mathbf{u v}}^{2}\left(\gamma_{\mathbf{v}}-1\right) \frac{1}{D_{\mathbf{u v}} \gamma_{\mathbf{u}}} \frac{(\mathbf{v} \cdot \mathbf{w})}{s^{2}}\right. \\
& +\frac{1}{s^{2}} \gamma_{\mathbf{u}} \gamma_{\mathbf{v}}{ }^{2} D_{\mathbf{u v}}\left(\gamma_{\mathbf{u}} \gamma_{\mathbf{v}} D_{\mathbf{u v}}+1\right)(\mathbf{v} \cdot \mathbf{w})+\frac{2}{s^{4}} \frac{\gamma_{\mathbf{u}}{ }^{2} \gamma_{\mathbf{v}}{ }^{4} D_{\mathbf{u v}}^{2}}{\left(\gamma_{\mathbf{v}}+1\right)} \frac{1+\gamma_{\mathbf{u}} D_{\mathbf{u v}}}{D_{\mathbf{u v}}\left(1+\gamma_{\mathbf{u}}\right)}(\mathbf{u} \cdot \mathbf{v})(\mathbf{v} \cdot \mathbf{w}) \\
& \left.+\frac{2}{s^{2}} \frac{\gamma_{\mathbf{u}}^{2} \gamma_{\mathbf{v}}^{2} D_{\mathbf{u v}}^{2}}{\left(\gamma_{\mathbf{v}}+1\right)} \frac{1}{D_{\mathbf{u v}} \gamma_{\mathbf{u}}} \frac{\gamma_{\mathbf{v}}^{2}-1}{\gamma_{\mathbf{v}}{ }^{2}}(\mathbf{v} \cdot \mathbf{w})\right\} \\
& =\frac{1}{D}\left\{-D_{\mathbf{u v}} \gamma_{\mathbf{v}}^{2}\left(1+\gamma_{\mathbf{u}} D_{\mathbf{u v}}\right) \frac{1}{s^{2}} \frac{\gamma_{\mathbf{u}}^{2}}{\gamma_{\mathbf{u}}+1}\left(\gamma_{\mathbf{v}}-1\right)(\mathbf{u} \cdot \mathbf{w})\right. \\
& +D_{\mathbf{u v}} \gamma_{\mathbf{v}}^{2}\left(1+\gamma_{\mathbf{u}} D_{\mathbf{u v}}\right) \frac{1}{s^{2}} \gamma_{\mathbf{u}} \gamma_{\mathbf{v}}(\mathbf{v} \cdot \mathbf{w}) \\
& \left.+D_{\mathbf{u v}} \gamma_{\mathbf{v}}^{2}\left(1+\gamma_{\mathbf{u}} D_{\mathbf{u v}}\right) \frac{2}{s^{4}} \frac{\gamma_{\mathbf{u}}^{2} \gamma_{\mathbf{v}}{ }^{2}}{\left(\gamma_{\mathbf{u}}+1\right)\left(\gamma_{\mathbf{v}}+1\right)}(\mathbf{u} \cdot \mathbf{v})(\mathbf{v} \cdot \mathbf{w})\right\} \\
& =D_{\mathbf{u v}} \gamma_{\mathbf{v}}{ }^{2}\left(1+\gamma_{\mathbf{u}} D_{\mathbf{u v}}\right) \frac{A}{D} \text {. }
\end{aligned}
$$


$D^{\prime}$ can be computed as in the following.

$$
\begin{aligned}
D^{\prime} & =\gamma_{\mathbf{u}} \gamma_{\mathbf{v}}^{2} D_{\mathbf{u v}}\left\{1+\frac{1+\gamma_{\mathbf{u}} D_{\mathbf{u v}}}{D_{\mathbf{u v}}\left(1+\gamma_{\mathbf{u}}\right)}\left(D_{\mathbf{u v}}-1\right)+\frac{1}{D_{\mathbf{u v}} \gamma_{\mathbf{u}}} \frac{\gamma_{\mathbf{v}}{ }^{2}-1}{\gamma_{\mathbf{v}}^{2}}\right\}+1 \\
& =\frac{\gamma_{\mathbf{u}} \gamma_{\mathbf{v}^{2}}\left(2 D_{\mathbf{u v}}-1+\gamma_{\mathbf{u}} D_{\mathbf{u v}}^{2}\right)}{1+\gamma_{\mathbf{u}}}+\gamma_{\mathbf{v}}^{2} \\
& =\frac{\gamma_{\mathbf{v}}^{2}\left(1+\gamma_{\mathbf{u}} D_{\mathbf{u v}}\right)^{2}}{1+\gamma_{\mathbf{u}}}
\end{aligned}
$$

Hence $\frac{A^{\prime}}{D^{\prime}} \frac{1+\gamma_{\mathbf{u}} D_{\mathbf{u v}}}{D_{\mathbf{u v}}\left(1+\gamma_{\mathbf{u}}\right)}=\frac{A}{D}$.

Next, we compute the coefficient of v. $B^{\prime}$ can be computed as in the following.

$$
\begin{aligned}
B^{\prime}= & -\frac{1}{s^{2}} \frac{\gamma_{\mathbf{v}}}{\gamma_{\mathbf{v}}+1}\left\{\gamma_{\mathbf{u}} \gamma_{\mathbf{v}} D_{\mathbf{u v}}\left(\gamma_{\mathbf{v}}+1\right) \frac{1+\gamma_{\mathbf{u}} D_{\mathbf{u v}}}{D_{\mathbf{u v}}\left(1+\gamma_{\mathbf{u}}\right.}(\mathbf{u} \cdot \mathbf{w})\right. \\
& \left.+\gamma_{\mathbf{u}} \gamma_{\mathbf{v}} D_{\mathbf{u v}}\left(\gamma_{\mathbf{v}}+1\right) \frac{1}{D_{\mathbf{u v}} \gamma_{\mathbf{u}}}(\mathbf{v} \cdot \mathbf{w})+\left(\gamma_{\mathbf{u}} \gamma_{\mathbf{v}} D_{\mathbf{u v}}-1\right) \gamma_{\mathbf{v}}(\mathbf{v} \cdot \mathbf{w})\right\} \\
= & -\frac{1}{s^{2}} \gamma_{\mathbf{v}}{ }^{2}\left(1+\gamma_{\mathbf{u}} D_{\mathbf{u v}}\right)\left\{\frac{\gamma_{\mathbf{u}}}{1+\gamma_{\mathbf{u}}}(\mathbf{u} \cdot \mathbf{w})+\frac{\gamma_{\mathbf{v}}}{1+\gamma_{\mathbf{v}}}(\mathbf{v} \cdot \mathbf{w})\right\} .
\end{aligned}
$$

The Equation (25) is rewritten by $A^{\prime} \frac{1}{D_{\mathbf{u v}} \gamma_{\mathbf{u}}}=\frac{A}{D} \frac{\gamma_{\mathbf{v}^{2}\left(1+\gamma_{\mathbf{u}} D_{\mathbf{u v}}\right)}}{\gamma_{\mathbf{u}}}$. Then

$$
\begin{aligned}
& A^{\prime} \frac{1}{D_{\mathbf{u v}} \gamma_{\mathbf{u}}}+B^{\prime} \\
= & \frac{\gamma_{\mathbf{v}}^{2}\left(1+\gamma_{\mathbf{u}} D_{\mathbf{u v}}\right)}{D}\left\{-\frac{1}{s^{2}} \frac{\gamma_{\mathbf{u}}}{\gamma_{\mathbf{u}}+1}\left(\gamma_{\mathbf{v}}-1\right)(\mathbf{u} \cdot \mathbf{w})+\frac{1}{s^{2}} \gamma_{\mathbf{v}}(\mathbf{v} \cdot \mathbf{w})\right. \\
& \left.+\frac{2}{s^{4}} \frac{\gamma_{\mathbf{u}} \gamma_{\mathbf{v}}{ }^{2}}{\left(\gamma_{\mathbf{u}}+1\right)\left(\gamma_{\mathbf{v}}+1\right)}(\mathbf{u} \cdot \mathbf{v})(\mathbf{v} \cdot \mathbf{w})-D\left(\frac{1}{s^{2}} \frac{\gamma_{\mathbf{u}}}{1+\gamma_{\mathbf{u}}}(\mathbf{u} \cdot \mathbf{w})+\frac{1}{s^{2}} \frac{\gamma_{\mathbf{v}}}{1+\gamma_{\mathbf{v}}}(\mathbf{v} \cdot \mathbf{w})\right)\right\} \\
= & \frac{\gamma_{\mathbf{v}}^{2}\left(1+\gamma_{\mathbf{u}} D_{\mathbf{u v}}\right)}{D\left(1+\gamma_{\mathbf{u}}\right)}\left\{-\frac{1}{s^{2}}\left(\gamma_{\mathbf{u}}\left(\gamma_{\mathbf{v}}-1\right)+\left(\gamma_{\mathbf{u}} \gamma_{\mathbf{v}} D_{\mathbf{u v}}+1\right) \gamma_{\mathbf{u}}\right)(\mathbf{u} \cdot \mathbf{w})\right. \\
& +\frac{1}{s^{2}}\left(\gamma_{\mathbf{v}}\left(1+\gamma_{\mathbf{u}}\right)-\left(\gamma_{\mathbf{u}} \gamma_{\mathbf{v}} D_{\mathbf{u v}}+1\right) \frac{\gamma_{\mathbf{v}}}{1+\gamma_{\mathbf{v}}}\left(1+\gamma_{\mathbf{u}}\right)\right)(\mathbf{v} \cdot \mathbf{w}) \\
& \left.+\frac{2}{s^{2}} \frac{\gamma_{\mathbf{u}} \gamma_{\mathbf{v}}{ }^{2}}{\left(\gamma_{\mathbf{v}}+1\right)}\left(D_{\mathbf{u v}}-1\right)(\mathbf{v} \cdot \mathbf{w})\right\} \\
= & \frac{\gamma_{\mathbf{v}}{ }^{2}\left(1+\gamma_{\mathbf{u}} D_{\mathbf{u v}}\right)}{D\left(1+\gamma_{\mathbf{u}}\right)}\left\{-\frac{1}{s^{2}} \gamma_{\mathbf{u}} \gamma_{\mathbf{v}}\left(1+\gamma_{\mathbf{u}} D_{\mathbf{u v}}\right)(\mathbf{u} \cdot \mathbf{w})\right. \\
& \left.+\frac{1}{s^{2}} \frac{\gamma_{\mathbf{v}}{ }^{2}}{1+\gamma_{\mathbf{v}}}\left(1+\gamma_{\mathbf{u}} D_{\mathbf{u v}}\right)\left(1-\gamma_{\mathbf{u}}\right)(\mathbf{v} \cdot \mathbf{w})\right\} \\
= & \frac{D^{\prime}}{D} B
\end{aligned}
$$

Hence $\frac{1}{D^{\prime}}\left(A^{\prime} \frac{1}{D_{\mathbf{u v}} \gamma_{\mathbf{u}}}+B^{\prime}\right)=\frac{B}{D}$. We conclude that $\operatorname{gyr}\left[\mathbf{u} \oplus_{\mathbf{E}} \mathbf{v}, \mathbf{v}\right] \mathbf{w}=\operatorname{gyr}[\mathbf{u}, \mathbf{v}] \mathbf{w}$ for all $\mathbf{u}, \mathbf{v}, \mathbf{w} \in \mathbb{V}_{S}$, so (G5) holds.

We conclude that $\left(\mathbb{V}_{s}, \oplus_{E}\right)$ is a gyrogroup. Finally, we prove that it is gyrocommutative.

We prove (G6). We prove that $\mathbf{a} \oplus \mathbf{b}=\operatorname{gyr}[\mathbf{a}, \mathbf{b}](\mathbf{b} \oplus \mathbf{a})$ for all $\mathbf{a}, \mathbf{b} \in \mathbb{V}_{s}$. Gyroautomorphic inverse property defined by Ungar in [1] (Definition 3.1, p. 51) is given by the equation

$$
\ominus_{E}\left(\mathbf{a} \oplus_{E} \mathbf{b}\right)=\ominus_{E} \mathbf{a} \ominus_{E} \mathbf{b},
$$


where $\mathbf{a}, \mathbf{b}$ are arbitrary elements in $\mathbb{V}_{s}$. According to Theorem 3.2 in [1] (p. 51), $\left(\mathbb{V}_{s}, \oplus_{E}\right)$ is gyrocommutative if and only if it has the gyroautomorphic inverse property. So, we observe the gyroautomorphic inverse property:

$$
\begin{aligned}
\ominus_{E}\left(\mathbf{a} \oplus_{E} \mathbf{b}\right) & =-\left(\mathbf{a} \oplus_{E} \mathbf{b}\right) \\
& =-\frac{1}{1+\frac{\mathbf{a} \cdot \mathbf{b}}{s^{2}}}\left\{\mathbf{a}+\frac{1}{\gamma_{\mathbf{a}}} \mathbf{b}+\frac{1}{s^{2}} \frac{\gamma_{\mathbf{a}}}{1+\gamma_{\mathbf{a}}}(\mathbf{a} \cdot \mathbf{b}) \mathbf{a}\right\}, \\
\ominus_{E} \mathbf{a} \ominus_{E} \mathbf{b} & =(-\mathbf{a}) \oplus_{E}(-\mathbf{b}) \\
& =\frac{1}{1+\frac{\mathbf{a} \cdot \mathbf{b}}{s^{2}}}\left\{-\mathbf{a}-\frac{1}{\gamma_{\mathbf{a}}} \mathbf{b}+\frac{1}{s^{2}} \frac{\gamma_{\mathbf{a}}}{1+\gamma_{\mathbf{a}}}(\mathbf{a} \cdot \mathbf{b})(-\mathbf{a})\right\} \\
& =\ominus_{E}\left(\mathbf{a} \oplus_{E} \mathbf{b}\right) .
\end{aligned}
$$

Hence $\left(\mathbb{V}_{s}, \oplus_{E}\right)$ is gyrocommutative.

Author Contributions: Conceptualization of gyrogeometric mean, T.H. The proof that the Einstein gyrogroup is a gyrogroup is by T.H. Except these all authors contributed equally to this article. All authors have read and agreed to the published version of the manuscript.

Funding: This research was funded by the Japan Society for the Promotion of Science: $19 K 03536$.

Conflicts of Interest: The authors declare no conflict of interest.

\section{References}

1. Ungar, A.A. Analytic Hyperbolic Geometry and Albert Einstein's Special Theory of Relativity; World Scientific Publishing Co. Pte. Ltd.: Hackensack, NJ, USA, 2008; 628p, ISBN 978-981-277-229-9. 981-277-229-4.

2. Suksumran, T.; Wiboonton, K. Einstein gyrogroup as a B-loop. Rep. Math. Phys. 2015, 76, 63-74. [CrossRef]

3. Bhatia, R.; Holbrook, J. Riemannian geometry and matrix geometric means. Linear Algebra Appl. 2006, 413, 594-618. [CrossRef]

4. Ando, T.; Li, C.-K.; Mathias, R. Geometric means. Linear Algebra Appl. 2004, 385, 305-334. [CrossRef]

5. Ungar, A.A. A gyrovector space approach to hyperbolic geometry. In Synthesis Lectures on Mathematics and Statistics; Morgan \& Claypool Publishers: Williston, VT, USA, 2009; 182p, ISBN 978-1-59829-822-2.

(C) 2020 by the authors. Licensee MDPI, Basel, Switzerland. This article is an open access article distributed under the terms and conditions of the Creative Commons Attribution (CC BY) license (http:/ / creativecommons.org/licenses/by/4.0/). 